\title{
THE DESIGN AND FABRICATION OF A MICROFLUIDIC REACTOR FOR SYNTHESIS OF CADMIUM SELENIDE QUANTUM DOTS USING SILICON AND GLASS SUBSTRATES
}

\author{
A Thesis \\ presented to \\ the Faculty of California Polytechnic State University, \\ San Luis Obispo, CA \\ in Partial Fulfillment \\ of the Requirements for the Degree \\ Masters of Science in General Engineering \\ With a Specialization in Materials Engineering \\ By \\ Peter Robert Gonsalves
}

February 27, 2012 
(C) 2012

Peter Robert Gonsalves

ALL RIGHTS RESERVED 
COMMITTEE MEMBERSHIP

TITLE: $\quad$ The Design and Fabrication of a Microfluidic Reactor for Synthesis of Cadmium Selenide Quantum Dots Using Silicon and Glass Substrates

AUTHOR: $\quad$ Peter Robert Gonsalves

DATE SUBMITTED: $\quad$ February 27, 2012

COMMITTEE CHAIR: $\quad$ Richard Savage, Ph. D

COMMITTEE MEMBER: Katherine Chen, Ph. D.

COMMITTEE MEMBER: David Clague, Ph. D. 


\title{
Abstract
}

\author{
THE DESIGN AND FABRICATION OF A MICROFLUIDIC REACTOR FOR \\ SYNTHESIS OF CADMIUM SELENIDE QUANTUM DOTS USING SILICON AND \\ GLASS SUBSTRATES \\ Peter Robert Gonsalves
}

A microfluidic reactor for synthesizing cadmium selenide (CdSe) quantum dots (QDs) was synthesized out of a silicon wafer and Pyrex glass. Microfabrication techniques were used to etch channels into the silicon wafer. Holes were wet-drilled into the Pyrex glass using a diamond-tip drill bit. The Pyrex wafer was anodically bonded to the etched silicon wafer to enclose the microfluidic reactor. Conditions for anodic bonding were created by exposing the stacked substrates to $300 \mathrm{~V}$ at $\sim 350^{\circ} \mathrm{C}$ under $5.46 \mathrm{~N}$ of force. A syringe containing a room temperature CdSe solution was interfaced to the microfluidic reactor by using Poly (dimethylsiloxane) (PDMS) as an interface. The reactor was placed on a hot plate at $225^{\circ} \mathrm{C}$, creating thermodynamic conditions for the QD chemical reaction to occur within the etched channels. Tygon ${ }^{\circledR}$ tubing transported solutions in and out of the microfluidic reactor. The CdSe solution was injected into the reactor by a syringe pump at an injection rate of $5 \mathrm{~mL} / \mathrm{hr}$, with a channel length of $2.5 \mathrm{~cm}$. While in the microfluidic channels, QD residence time of approximately 30 seconds was sufficient enough for nucleation and growth of QDs to occur. The QD size was characterized by fluorescence full-width-half-maximum (FWHM), which is directly proportional to size distribution. The FWHM of the QDs synthesized was $38 \mathrm{~nm}$, with a peak wavelength of $492 \mathrm{~nm}$. By controlling combinations of pump rate and channel length, a range of QD sizes was able to be consistently synthesized through the microfluidic reactor with significant repeatability and reproducibility. 


\section{Acknowledgments}

I would like to thank the following people for making my thesis project possible.

- Boeing for project funding

- General LED for project funding

- Dr. Richard Savage (Materials Engineering) for guidance and experience

- My family for their support during my long hours in the lab and late nights doing research. 


\section{Table of Contents}

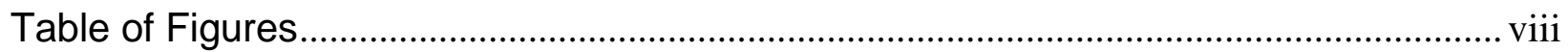

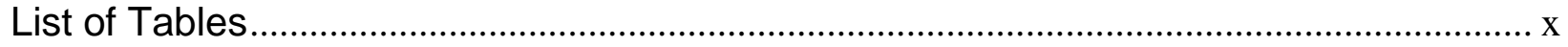

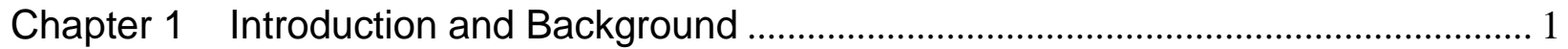

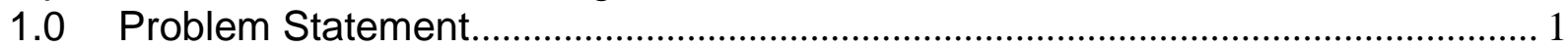

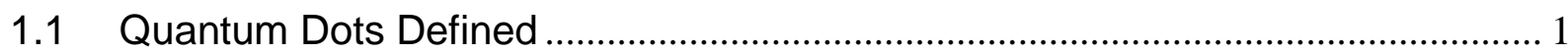

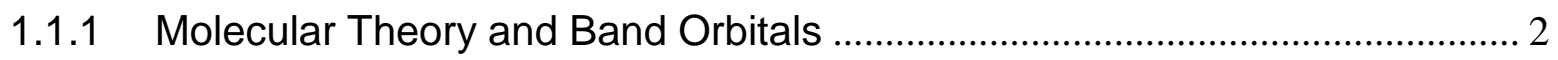

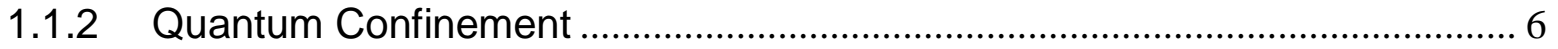

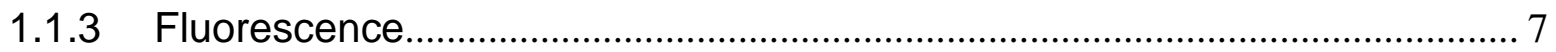

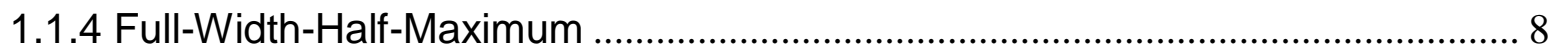

1.2 Quantum Dot Synthesis................................................................................... 9

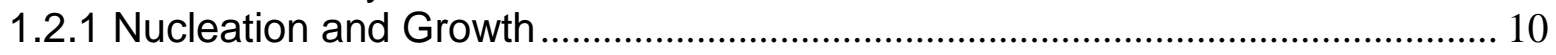

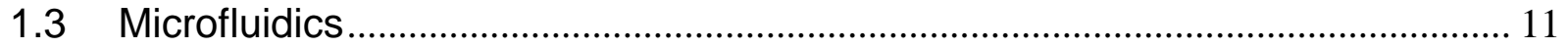

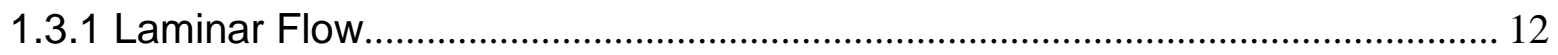

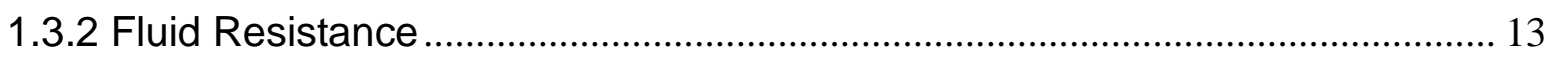

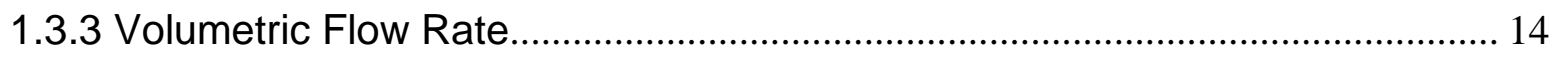

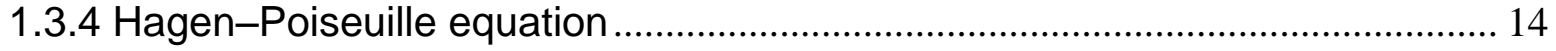

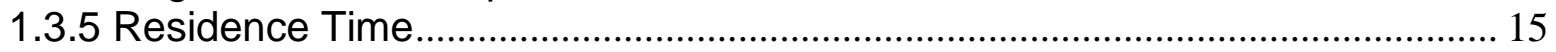

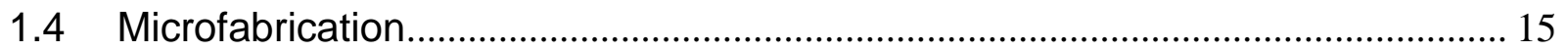

1.4.1 Physical Vapor Deposition (Sputtering) ........................................................... 16

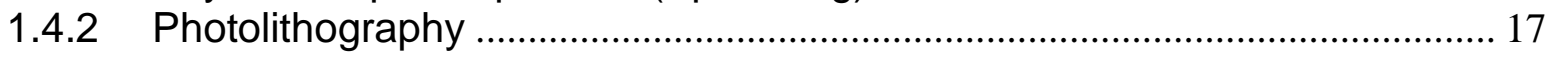

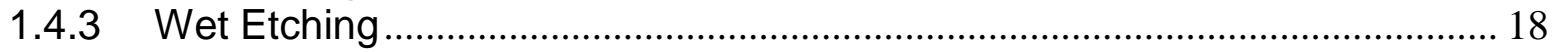

1.4.4 Reactive lon Etching (Dry Etching) ……..................................................... 19

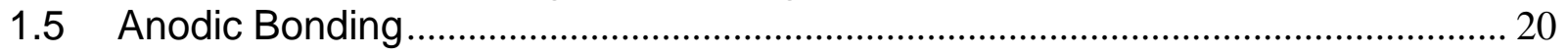

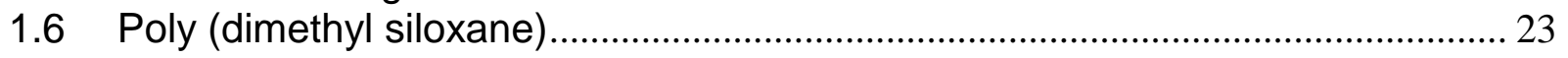

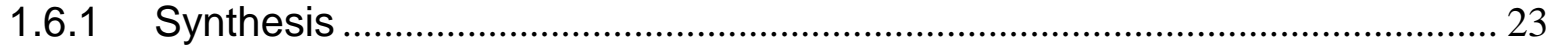

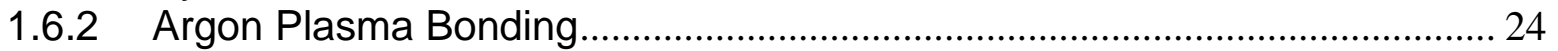

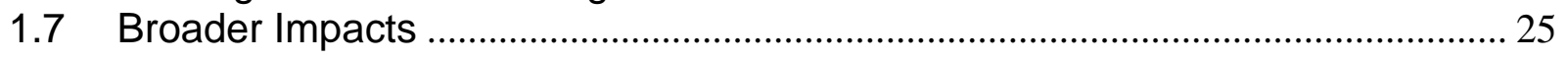

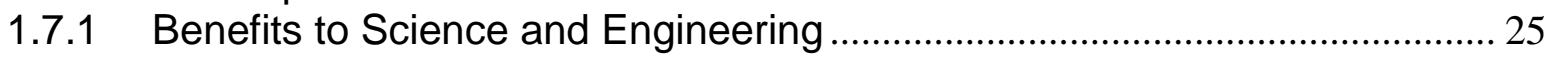

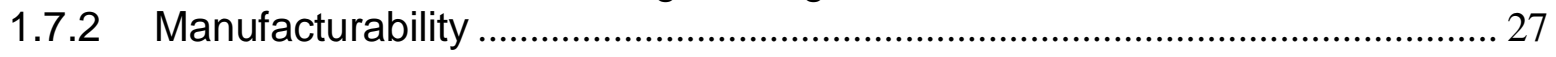

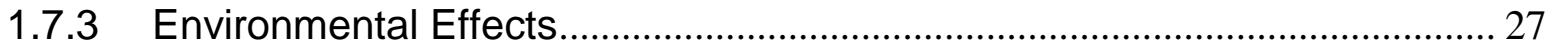

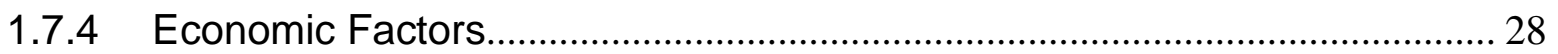

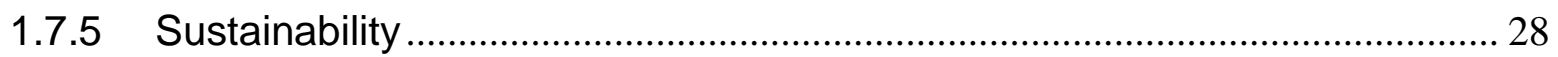

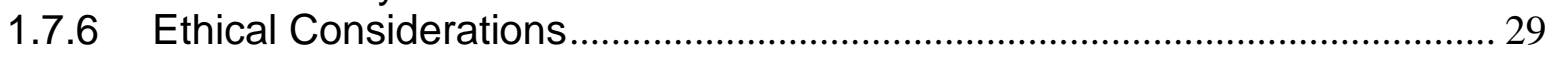

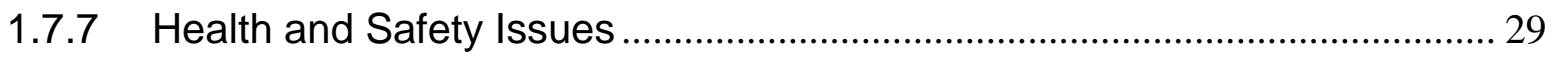

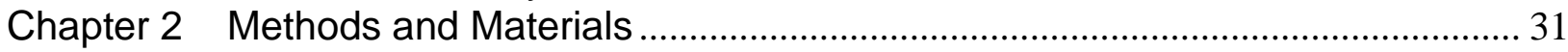

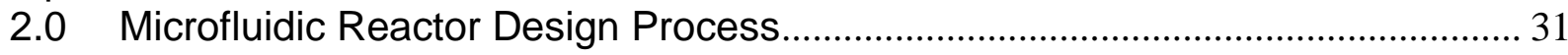

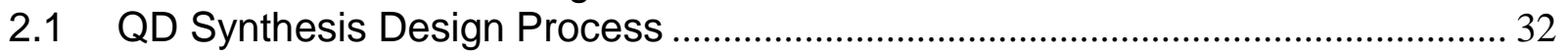

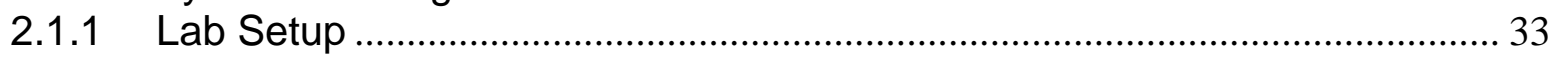

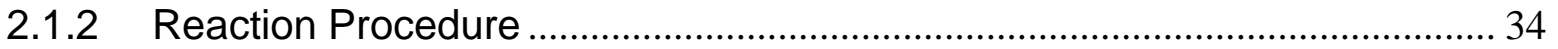

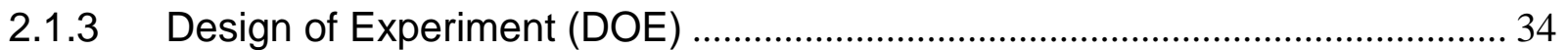

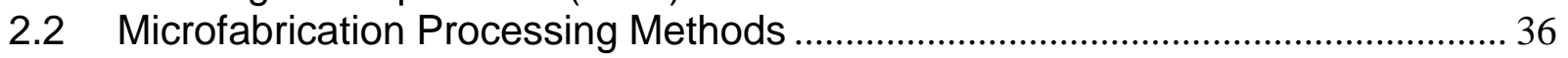

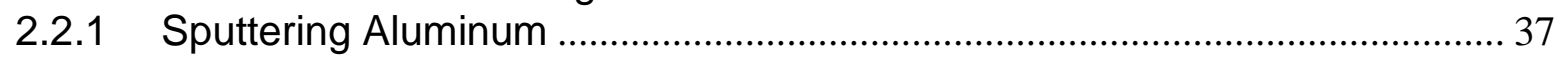

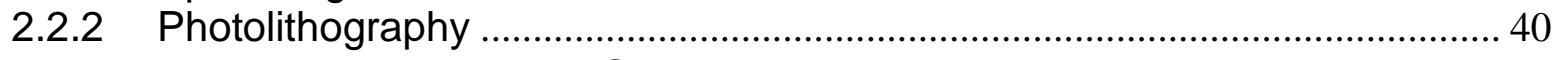

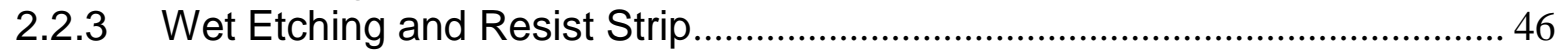

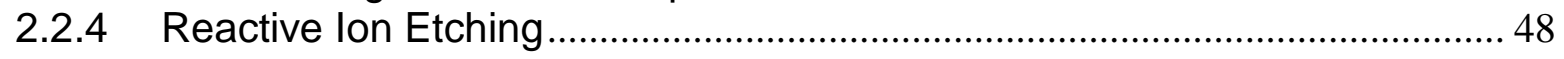




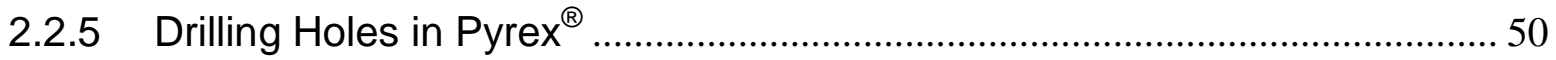

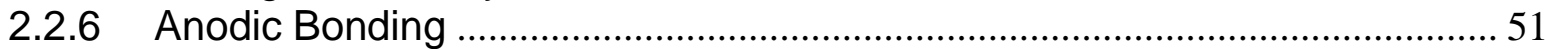

2.2.7 Interfacing Syringes to the Microfluidic Reactor ............................................5 53

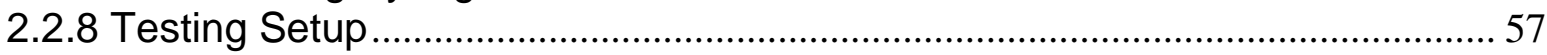

2.3 Characterization of Synthesized Quantum Dots .................................................... 60

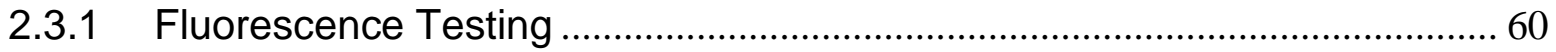

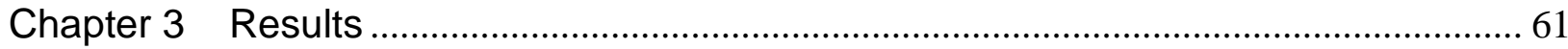

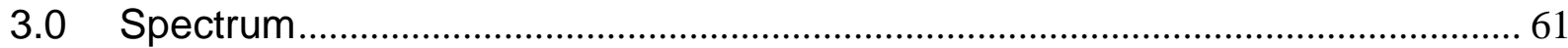

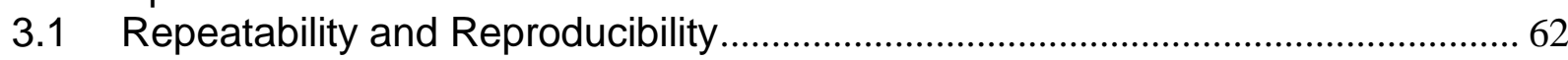

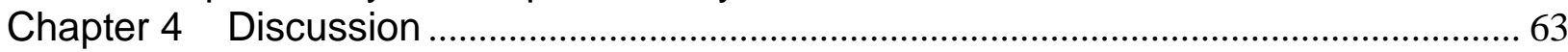

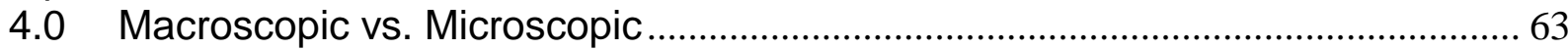

4.0.1 Can the Microfluidic Reactor Synthesize Blue or Red Light? ........................ 64

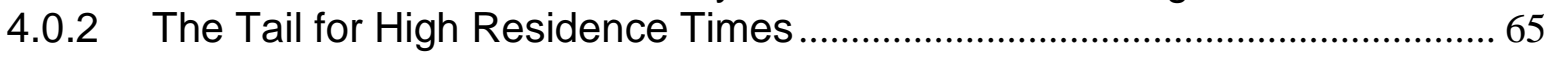

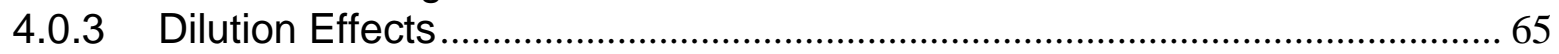

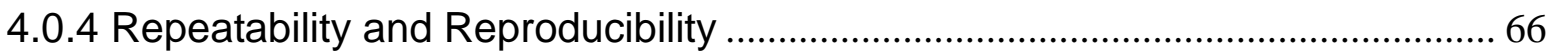

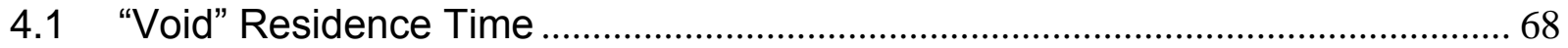

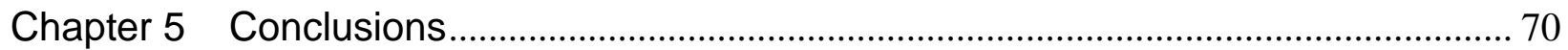

Chapter 6 Future Work and Recommendations ....................................................... 72

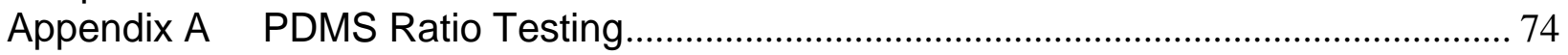

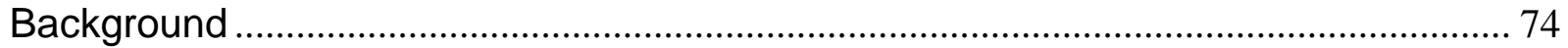

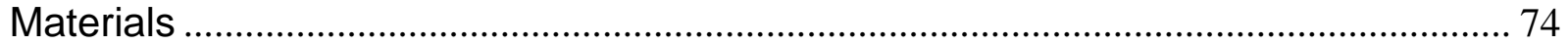

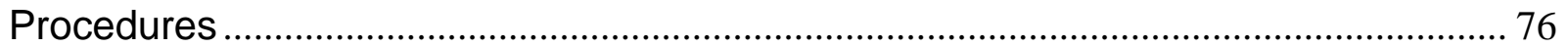

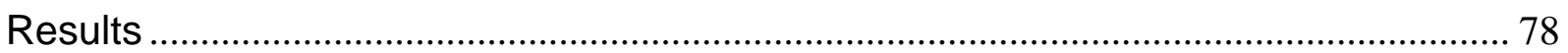

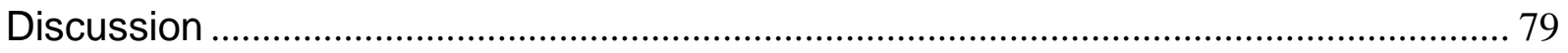

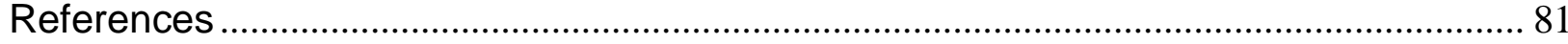




\section{Table of Figures}

Figure 1 - Fluorescence spectrum of quantum dot emissions.................................... 2

Figure 2 - Constructive/Destructive interference of hydrogen bonding .......................... 3

Figure 3 - Molecular orbital energy diagram....................................................... 4

Figure 4 - Evolution of molecular orbitals into electronic energy bands ........................ 5

Figure 5 - The Exciton Bohr radius .................................................................. 6

Figure 6 - The effects of quantum confinement for planes, wires, and dots .................. 7

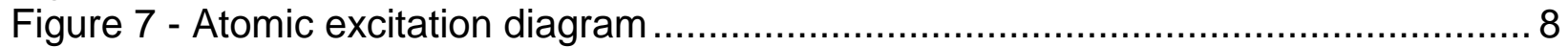

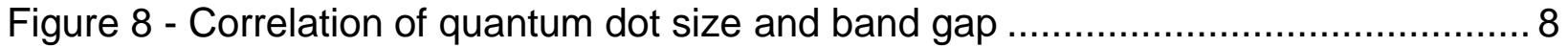

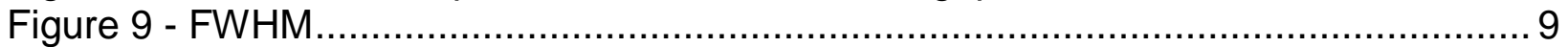

Figure 10 - Photolithography mask of microfluidic channels ..................................... 17

Figure 11 - Photolithography light integral plot .................................................... 18

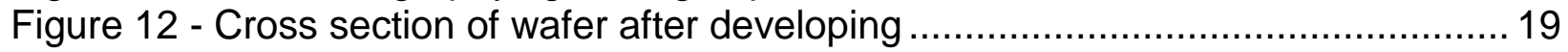

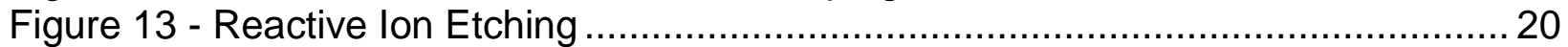

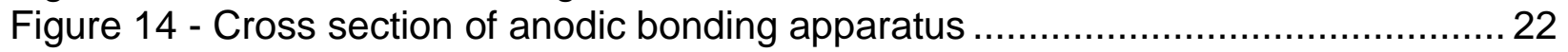

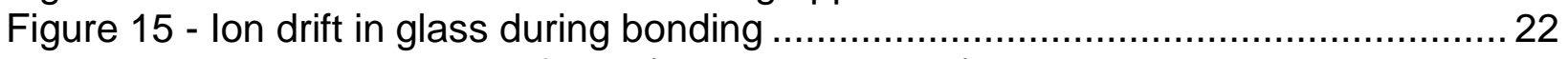

Figure 16 - Repeat structure of poly (dimethyl siloxane) ….....................................2 23

Figure 17 - Covalent bonding between plasma treated PDMS surfaces ...................... 24

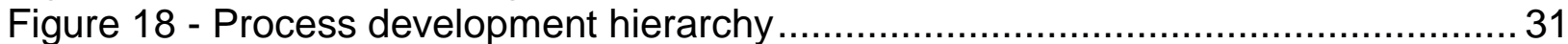

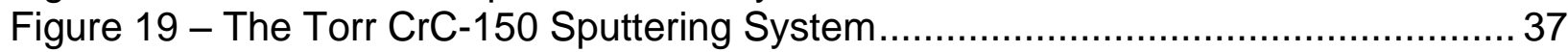

Figure 20 - Cross section of wafer after aluminum sputtering …............................... 39

Figure 21 - CRC-150 Sputtering System in action ............................................... 40

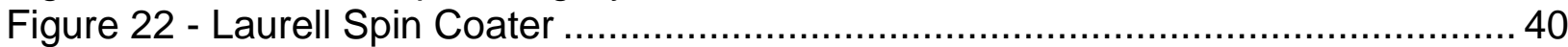

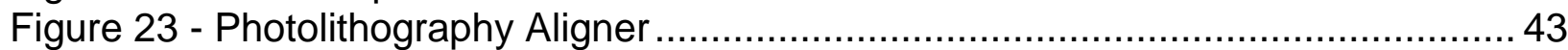

Figure 24 - Cross section of wafer during photolithography ..................................... 43

Figure 25 - Photolithography mask of microfluidic channels................................... 44

Figure 26 - Cross section of wafer after developing .............................................. 46

Figure 27 - Cross section of wafer after aluminum etching ................................... 47

Figure 28 - Cross section of water after resist strip ................................................ 48

Figure 29 - Cross section of wafer after Reactive Ion Etching................................... 50

Figure 30 - Cross section of wafer after stripping aluminum ................................... 50

Figure 31 - Alignment of Pyrex® wafer over the silicon wafer .................................. 50

Figure 32 - Cross section of drilling holes through glass ........................................51

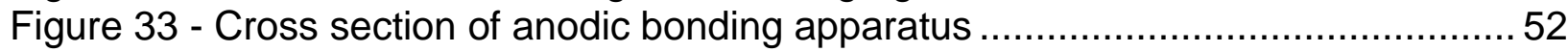

Figure 34 - Duradyne argon plasma system ………............................................ 53

Figure 35 - SS316 tubing "punched" through PDMS ............................................5 54

Figure 36 - SS316 Tubing inserted through the center of a PDMS block .................... 55

Figure 37 - Argon plasma processing PDMS and glass .......................................56

Figure 38 - Cross section of PDMS and microfluidic reactor ...................................5 57

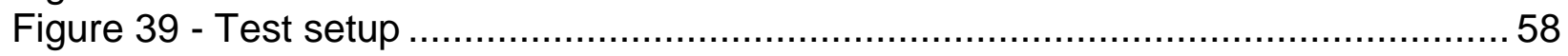

Figure 40 - Microfluidic device on the hotplate during testing..................................58

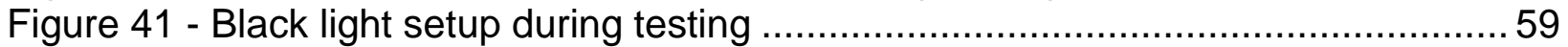

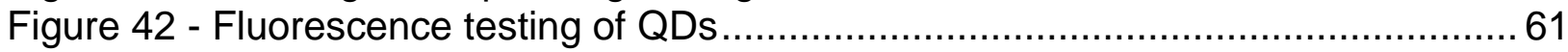


Figure 43 - Spectral profiles of QDs

Appendix Figures:

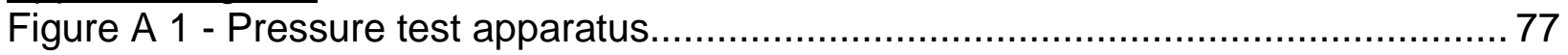

Figure A 2 - Paper towels over test apparatus.... 


\section{List of Tables}

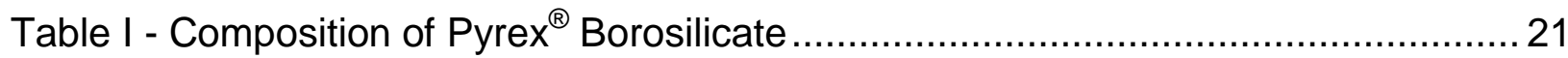

Table II - Parameters for Sputtering Aluminum on Silicon Wafer ................................. 39

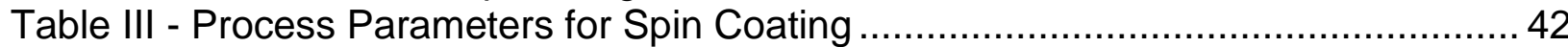

Table IV - Process Parameters for Photo Alignment .............................................. 46

Table V - Process Parameters for Developing Exposed Positive Photoresist ............... 46

Table VI - Process Parameters for Aluminum Etching ........................................... 47

Table VII - Process Parameters for Stripping Positive Resist..................................... 48

Table VIII - Process Parameters for Reactive Ion Etching ........................................ 49

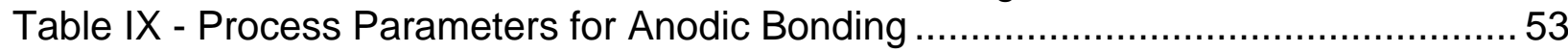

Table X - Repeatability and Reproducibility Tests Results ..........................................6 63

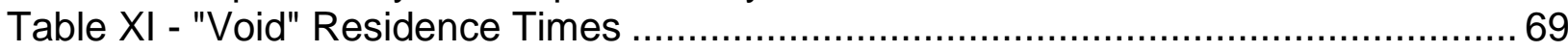

Table A I - Tested Mixture Ratios of PDMS Base to Curing Agent............................. 76

Table A II - Bursting Strengths of PDMS to Glass with Different PDMS Mixture Ratios 78 


\section{Chapter 1 Introduction and Background}

\subsection{Problem Statement}

The current process of fabricating quantum dots in the California Polytechnic (Cal Poly) State University Nanotechnology Lab is on the bulk scale (10-15 mL). Synthesis on the bulk scale produces a relatively broad $(30 \mathrm{~nm})$ spectrum of nanoparticles. Particle sizes are characterized by their full-width-half-maximum fluorescence spectral profile. There is a need to design a process for synthesizing quantum dots with a tighter size distribution $(<30 \mathrm{~nm})$. A microfluidic reactor will synthesize quantum dots under carefully controlled conditions and will produce a more discrete spectral profile. My goal is to create a microfluidic reactor capable of synthesizing quantum dots by using silicon and glass substrates.

\subsection{Quantum Dots Defined}

Quantum dots are semiconductor crystals made up of hundreds of atoms that are typically $2-10 \mathrm{~nm}$ in diameter. Due to their small size, quantum dots display properties that combine classical and quantum physics. The combination of behaving like a bulk material, while preserving characteristics of individual atoms make quantum dots unique because their properties change simply by altering their size [1].The optical properties of quantum dots are indicative of their size (Figure 1). 


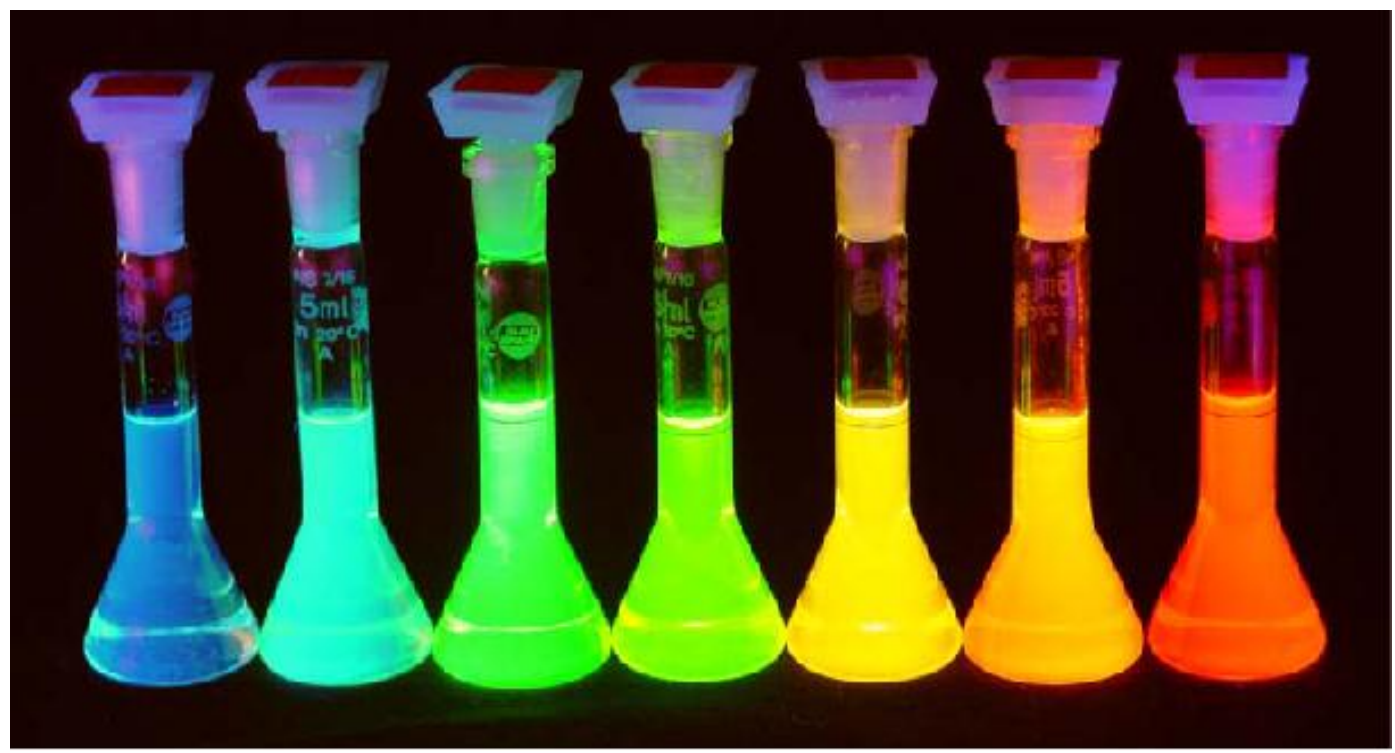

Figure 1 - A spectrum of quantum dot emissions, with quantum dot size increasing from left to right (band gap decreasing) [2].

Understanding why and how quantum dots behave the way they do begins with the electronic structure of the atoms that make up the quantum dots.

\subsubsection{Molecular Theory and Band Orbitals}

All atoms are basically composed of positively charged nuclei, surrounded by a negatively charged electron cloud. The Lewis Structure approach provides a simple method for determining the electronic structure of many molecules. A more general, but slightly more complicated approach is the Molecular Orbital (MO) Theory, which builds on the electron wave functions of quantum mechanics to describe chemical bonding. MO Theory suggests that electrons exist in energy levels called orbitals. The orbitals are thought of as shells that surround the nucleus. Shells that are closest to the nucleus are at a lower energy state than shells that are further away. Electrons within an atom often move to different orbitals to keep the atom in its lowest (favorable) energy state. 
One of the fundamental rules governing the mechanics of MO theory is that atomic orbitals are combined to create molecular orbitals; the number of molecular orbitals formed equals the number of atomic orbitals used [3]. Hydrogen, for example, is the simplest of all molecules. In its atomic form, hydrogen has only a single orbital (1s) with a single electron (Figure 2).

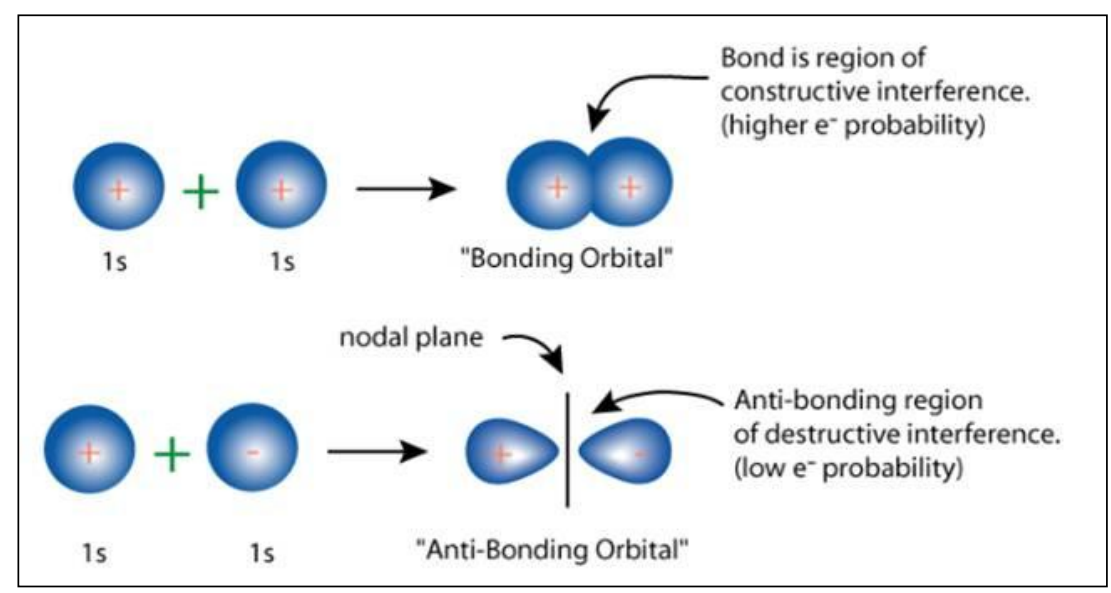

Figure 2 - The bonding of 2 hydrogen atoms is either constructive (bonding) or destructive (anti-bonding) interference [3].

The energy of an $\mathrm{H}_{2}$ molecule with 2 electrons in the bonding orbital is less than the combined energies of the 2 separate hydrogen atoms. Conversely, the energy of the $\mathrm{H}_{2}$ molecule with the 2 electrons in the anti-bonding orbital is higher than the combined energies of the 2 separate hydrogen atoms (Figure 3). 


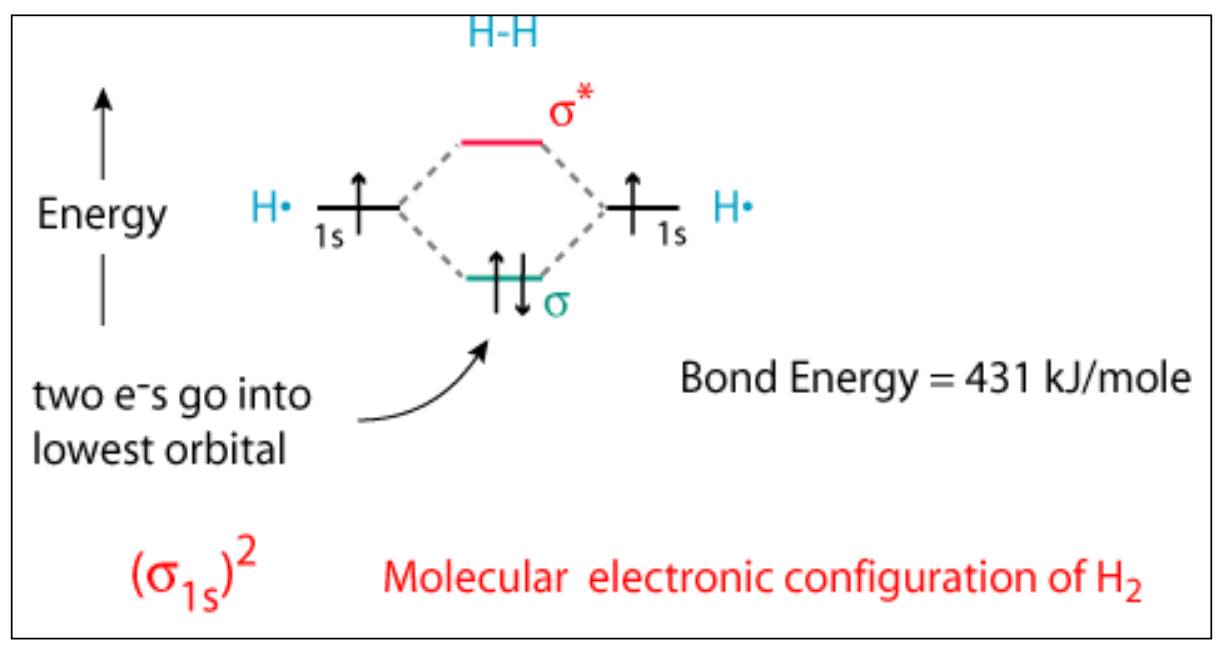

Figure 3 - Molecular orbital energy diagram. When two atomic orbitals combine to form two molecular orbitals, the orbital energies shift. The net change in energy is the same, but now there is a low energy and high energy orbital. The electrons move into the more stable, low energy, bonding orbital [3].

There is a greater probability that the 2 electrons from the original system will move to occupy the bonding orbital because it creates a lower energy state, which is preferred by nature since the molecule decreases in energy [4].

When this model is extended out to materials with more than two atoms, the number of available orbitals also increases, causing the orbital energies to shift (Figure 4). The end result is "energy" between levels so small it can be treated as a continuous band. Thus, the bonding orbital becomes the valence band, while the antibonding orbital becomes the conduction band [5]. 


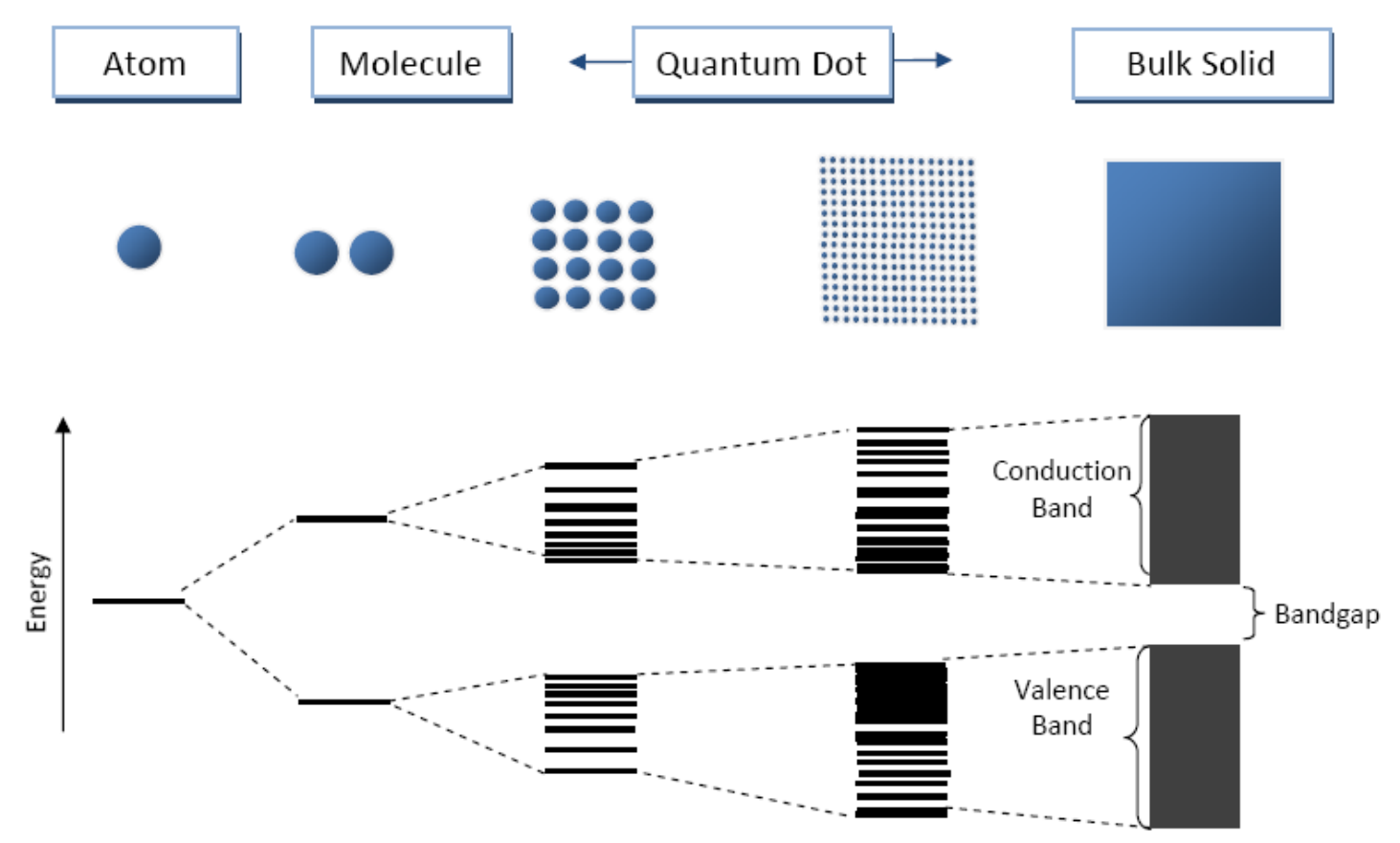

Figure 4 - Evolution of molecular orbitals into electronic energy bands. The point at which the discrete energy levels become a continuous band is where quantum dots cease being quantum and become a bulk solid.

The energy gap between the valence and conduction bands is called the band gap [4]. Electrons are unable to occupy the band gap region. The point where the discrete energy levels become a continuous band is the point where a quantum dot is considered a bulk solid, at approximately $10 \mathrm{~nm}$ in size.

The only way an electron in the valence band of a natural bulk semiconductor can jump the band gap to the conduction band is to acquire enough energy to do so. In a bulk material, this is not possible without the help of an outside stimulus, such as heat or an applied voltage. Due to the size of quantum dots, an excitation source such as a high energy photon (light) can induce electrons to jump the gap to the conduction band. The excited electron now in the conduction band and the "hole" it left behind in the valence 
band are considered an exciton pair (Figure 5). The physical distance between them is called the Exciton Bohr radius [6].

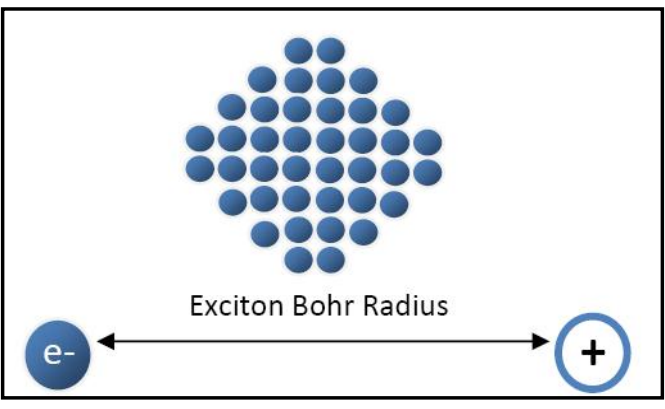

Figure 5 - The Exciton Bohr radius is the distance between an excited electron and the hole that it left behind when jumping the band gap. The particle depicted here is smaller than this distance so it experiences a phenomenon known as quantum confinement.

In a bulk material, the Exciton Bohr radius is much smaller than the size of the material itself, so the radius can extend to its full natural limit; however, in a quantum dot the Exciton Bohr radius is close to or larger than the material particle. This occurs around $10 \mathrm{~nm}$ and the resulting exciton pairs are limited by the size of the material. Excited electrons cannot move to their full natural radius because the surface of the quantum dot is holding them back, which is an effect called quantum confinement.

\subsubsection{Quantum Confinement}

In bulk materials, the number of energy states available to the electrons is a virtually infinite logarithmic curve. Only as the dimensions of the material are reduced to that below the Exciton Bohr radius do we see certain energy states become unavailable. In 2D films, the excitons can extend fully in 2 directions, which are called quantum planes. Quantum wires further restrict the number of available energy states, limiting excitons to extend in only one direction. When the entire material is smaller than the Exciton Bohr 
radius in all directions, only discrete energy levels remain, which is the definition of a quantum dot [Figure 6] [6].

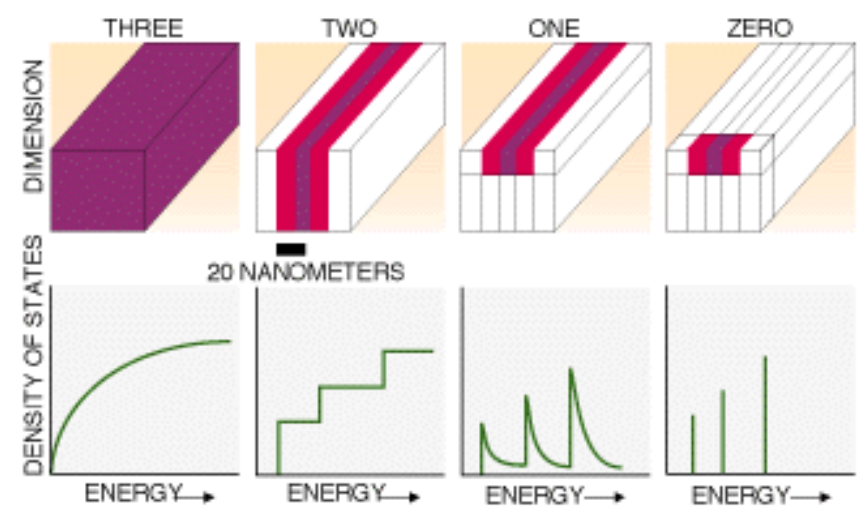

Figure 6 - Diagram showing the effects of quantum confinement for planes, wires, and dots [7]. Due to quantum confinement, adding or removing a single orbital impacts the total energy level of the system. As atoms are added, energy levels are also added to the top of the valence band and the bottom of the conduction band, resulting in a decrease in the total size of the band gap. Controlling the size of the quantum dot correlates to tuning the size of the band gap [6].

\subsubsection{Fluorescence}

Fluorescence is the emission of light by a substance that has absorbed light, which is the unique material property of quantum dots that makes them so desirable. The color seen in a bulk material is the result of an excited electron jumping up to the conduction band, and then immediately after jumping to the conduction band, the electron falls back down to the valence band and emits a photon with energy equal to the band gap of the material (Figure 7). 

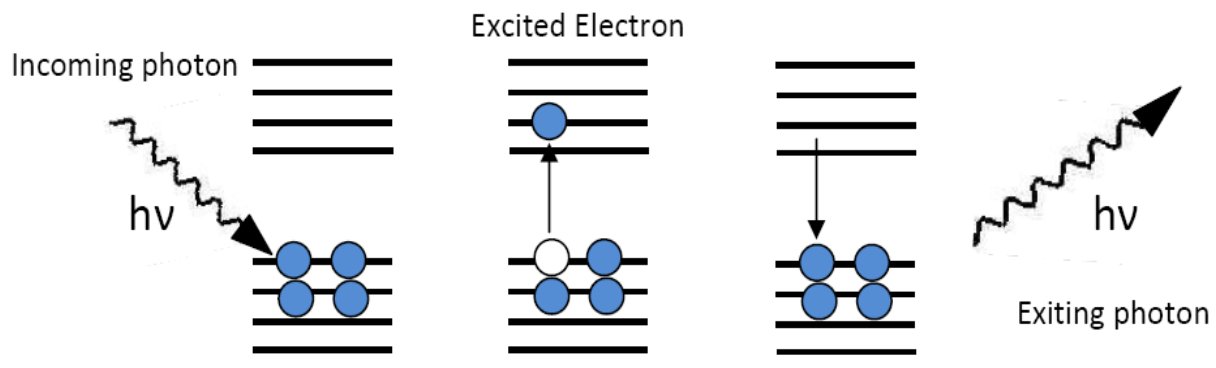

Figure 7 - Process of exciting an atom with a high energy photon and releasing a lower energy photon back out.

The same process holds true with quantum dots; however, since the band gaps of quantum dots can be altered with an increase or decrease in size, it is possible to change the color of the emitted photons [7]. This effect can be summarized in Figure 8.

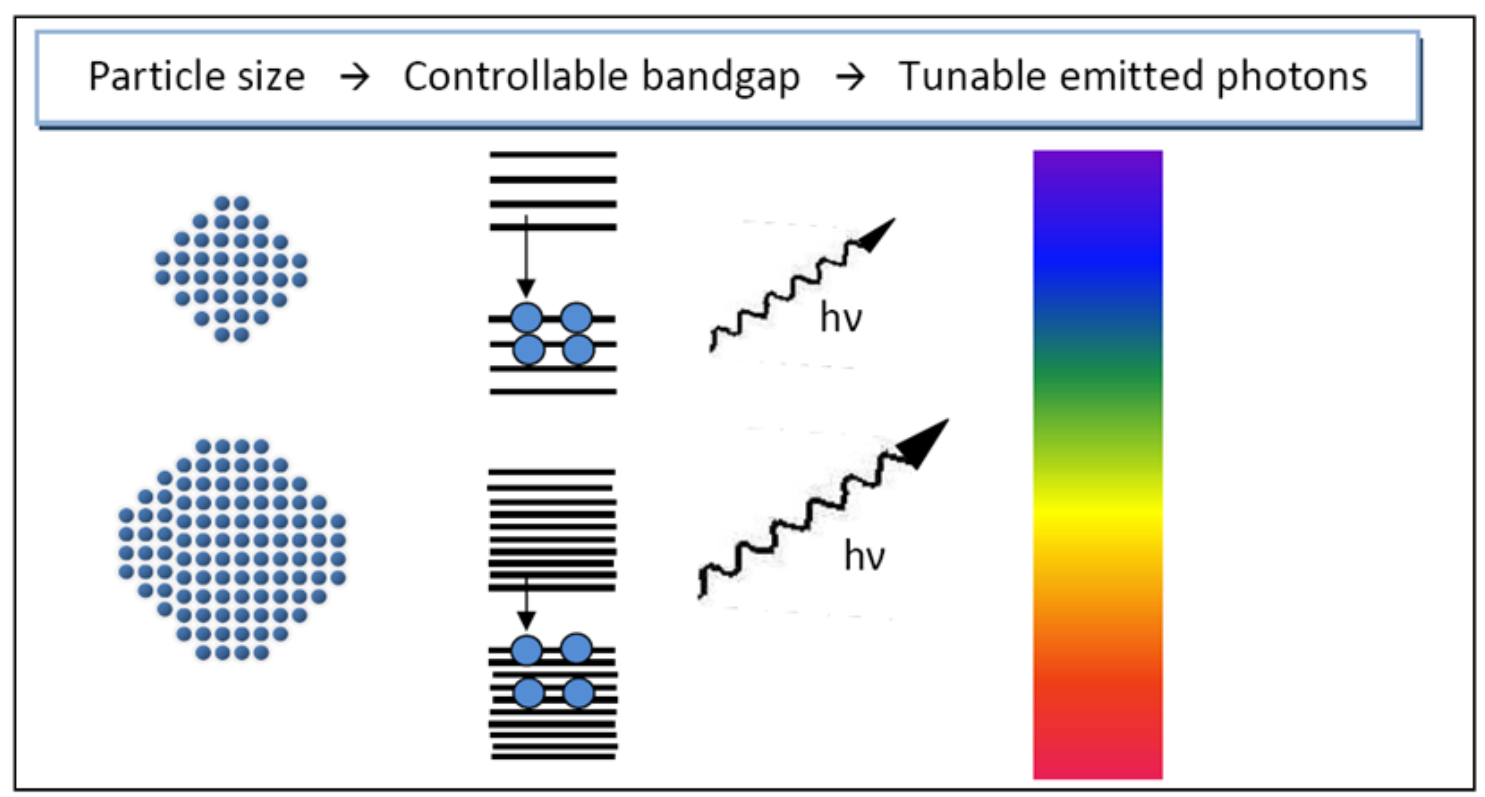

Figure 8 - Diagram showing the direct correlation of quantum dot size leading to different sized band gaps and different wavelengths of emitted photons.

\subsubsection{Full-Width-Half-Maximum}

Irradiating a QD sample with UV light generates a fluorescent spectral profile. An ideal spectral profile is a vertical line at a given wavelength indicating that the entire synthesis of quantum dots is the same size; however, a typical quantum dot synthesis is not 
perfect. Typical fluorescent profiles are curves that resemble normal distribution curves; therefore, the best method for characterizing fluorescence spread is by a method called the full-width-half-maximum (FWHM) (Figure 9).

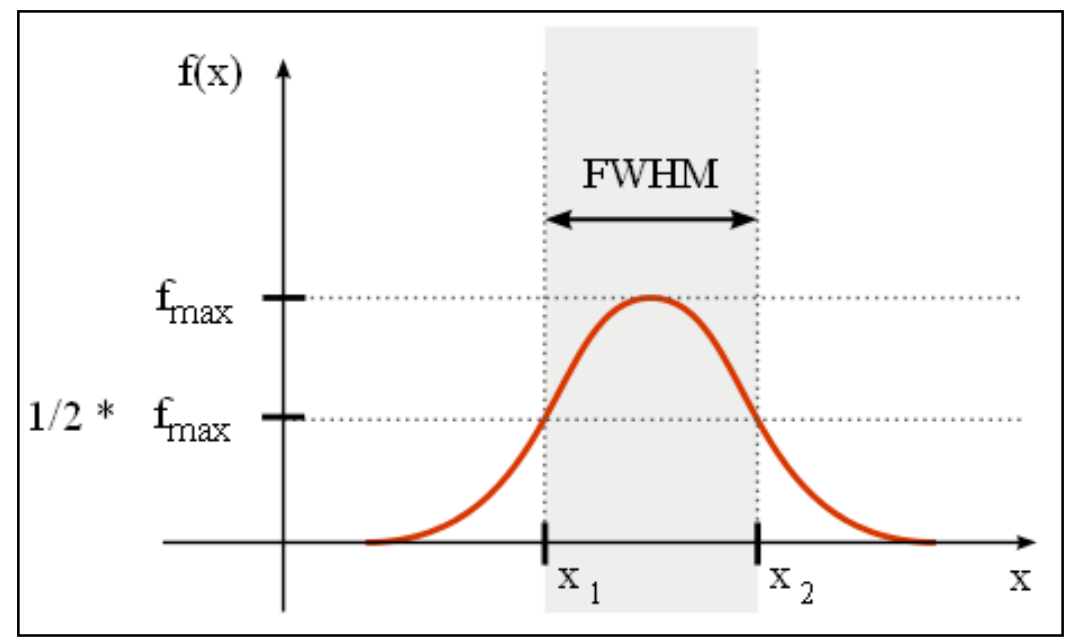

Figure 9 - The FWHM is determined as the difference in $x$ values at half the maximum $y$ value [8].

To measure the FWHM, first the greatest peak value $\left(f_{\max }\right)$ is identified. Then, half this peak intensity value is determined $\left(1 / 2{ }^{*} f_{\max }\right)$. Next, a horizontal line is drawn through the curve such that it intersects at two distinct points $\left(\mathrm{x}_{1}\right.$ and $\left.\mathrm{x}_{2}\right)$. Finally, the FWHM is determined as the difference between the two $x$ values $\left(x_{2}-x_{1}\right)[8]$.

\subsection{Quantum Dot Synthesis}

Quantum dot synthesis begins with the synthesis of two precursor solutions, one containing a selenium compound; the other containing dissolved cadmium ions. Mixed together at temperatures above $180^{\circ} \mathrm{C}$ results in an oxidation-reduction reaction whereby crystals of cadmium selenide (CdSe) nucleate and grow [5]. Extraction and cooling of samples from the reaction vessel at different time intervals halts the reaction; thus allowing some level of control over the spectra of particle sizes synthesized. 
Aaron Lichtner designed a process of synthesizing quantum dots at Cal Poly on the bulk scale $(\sim 15-20 \mathrm{~mL})$, based on other work [9]. Similar processes have been developed on the micron scale by controlling the flow of the precursor solutions through microfluidic channels. These microfluidic reactors were placed over heat sources that allowed the reaction to occur within channels. Control over fluid velocity translates into control over CdSe residence time, which leads to control over QD size distribution [10].

\subsubsection{Nucleation and Growth}

During solidification, solid precipitates appear at random sites throughout the liquid, as well as along the walls of the containment vessel. The interface between solid and liquid allows migration of atoms from one phase to the other. These two stages are called nucleation and growth. The size of the nuclei spontaneously formed is a statistical phenomenon, but it depends on the same factors as growth rate (the driving force and the diffusion rate), the temperature of the reaction and the time allowed [11].

There are two types of nucleation: homogeneous and heterogeneous. The spontaneous formation of new phases, such as solid crystals within the bulk of a liquid, is strictly known as homogeneous nucleation. Alternatively, nature favors phase formations that begin by attaching to interfaces within the system (such as the walls of a microfluidic channel), which is called heterogeneous nucleation [12]. Both homogeneous and heterogeneous nucleation and growth mechanisms occur simultaneously during normal growth reactions, including the flow through microfluidic channels. 
The temperature has the greatest impact on the amount of nucleation, whereas the time has the greatest impact on the amount of growth of the particles in the reaction. Low temperatures and long times will result in large particle sizes (approximately 8-10nm in size). Conversely, high temperatures and short times will result in small particles sizes $(2-4 \mathrm{~nm}$ in size). The key to synthesizing a desirable concentration of quantum dots through the microfluidic reactor is to experimentally determine the ideal combination of temperature and residence time.

The research carried out through this project was not intended on identifying the relationships between time, temperature and quantum dot size. Rather, temperature and time were held constant such that the reliability of the microfluidic channels was investigated based on the repeatable and reproducible size of the quantum dots.

\subsection{Microfluidics}

A microfluidic reactor is a device that allows chemical reactions to occur in confined channels, with dimensions below $1 \mathrm{~mm}$ [13]. Microfluidic reactors are designed for continuous flow and offer many advantages over conventional bulk scale reactions. Typical advantages include improvements in energy efficiency, reaction speed, yield, safety, reliability, scalability, on-site/on-demand production, and a finer degree of process control. Microfluidic reactors are typically made using polymers (such as poly (dimethyl siloxane), but my thesis will focus on making the microfluidic reactor by etching a silicon wafer and bonding to glass. The main reason for choosing non-polymer substrate materials is because polymers like PDMS are not suitable for high- 
temperature quantum dot synthesis, nor are they are compatible with the solvents commonly associated with quantum dot synthesis, such as octadecene.

\subsubsection{Laminar Flow}

Laminar flow occurs when two fluids flow together in parallel layers with no disruption between the layers. The equation that determines whether or not a system will experience laminar flow is called the Reynolds Number [14],

$$
R_{e}=\frac{\rho \boldsymbol{V D}}{\mu_{d}}
$$

where $\rho$ is the density of the liquid, $v$ is the velocity, $D$ is the hydraulic diameter, and $\mu_{d}$ is the dynamic viscosity of the liquid. In this project, the channel shape is rectangular, so $D$ is calculated as:

\section{$2 a b /(a+b)$}

where $a \& b$ are the sides of a rectangular-shaped channel. The Reynolds Number is a dimensionless number that gives a measure of the ratio of inertial forces to viscous forces. A micro-electrical-mechanical-system (or MEMS) device exhibits laminar flow when it has a Reynolds number below 1 . The microfluidic reactor fabricated in this project has a Reynolds Number many orders of magnitude below 1; thus, laminar flow is exhibited and taken into account.

Originally, a Cal Poly student designed a QD microfluidic reactor to mix the cadmium and selenide precursors within the microfluidic channels [15]. The mask was designed 
to account for laminar flow by incorporating several sharp $\left(180^{\circ}\right)$ turns within the channels that would mix the solution through a layer-folding mechanism; however, it proved difficult to control the flow rates of the two solutions simultaneously to encourage mixing; thus, I investigated if two separate solutions were needed.

Testing the bulk cadmium and selenide precursors mixed at room temperature (RT) revealed no obvious reaction; thus, for the purposes of this project they were treated as a single solution. The RT CdSe solution lacks sufficient heat for a rapid nucleation and growth reaction; thus, as long as the solution is used within a few hours of being synthesized, there is negligible impact on the microfluidic nucleation and growth reaction.

\subsubsection{Fluid Resistance}

Pressure builds quickly in a microfluidic device; therefore, it is essential to evaluate the variables that cause device failure as a result of pressure problems. The two major factors creating pressure in microfluidic reactors are fluid resistance and volumetric flow rate. Fluid resistance is an indicator of the shear forces the walls of the channel exert on the liquid flowing through them. For rectangular channels, fluid resistance is calculated by:

$$
R=\frac{12 \mu_{d} L}{w h^{3}}
$$


where $L$ is the length of the channel, w is the width of the channel, and $h$ is the etch depth of the channel [16]. Pressure is minimized by having shorter channel lengths, larger channel widths, and deeper channel etch depths.

\subsubsection{Volumetric Flow Rate}

The other factor of pressure is the volumetric flow rate, which is a function of the syringe pump rate [16]. Volumetric flow rate $(Q)$ is calculated by:

$$
\mathrm{Q}\left(\mathrm{m}^{3} / \mathrm{s}\right)=v(\mathrm{~m} / \mathrm{s}) * A\left(\mathrm{~m}^{2}\right)
$$

where $\mathrm{v}$ is the velocity of the fluid, and $A$ is the cross-sectional area of a microfluidic device, which remains constant (though unique) for each device. Typically, the pump rate is the variable that dictates adjustments to volumetric flow rate.

\subsubsection{Hagen-Poiseuille equation}

The total pressure in the channels is calculated by using the Hagen-Poiseuille equation, which is the product of fluid resistance $\left(\mathrm{Ns} / \mathrm{m}^{5}\right)$ and volumetric flow rate $\left(\mathrm{m}^{3} / \mathrm{s}\right)[16]$ :

$$
\Delta P_{\mu}=R * Q_{\text {flow rate }}
$$

The pressure forces occur as a necessary balance to the viscous forces due to the shear stresses on the channel walls. All factors are considered so that variations in etch depth (h), pump rate (velocity), and length (L) can be made to accommodate and limit the pressure inside the microfluidic reactor. 


\subsubsection{Residence Time}

Controlling quantum dot size during the bulk scale synthesis is accomplished by controlling the temperature and time allowed for the CdSe nucleation and growth reaction. On the bulk synthesis scale, the reaction is timed from the point that the solutions are injected into a heated flask to the point that the solution is extracted (via syringe). Upon extraction, the temperature drops quickly and the reaction slows down significantly enough to be considered halted.

In the microfluidic reactor, the time the fluid is running through the channels is called the residence time. A simple calculation between the velocity of the fluid and the distance the fluid travels through the channel gives an approximate residence time; however, the channel volume is considerably smaller than the Tygon ${ }^{\circledR}$ outlet tubing, specifically 350

times smaller. As a result, the fluid actually "resides" within the microfluidic reactor for a longer period of time than what is determined from the calculations (usually about 1 second in microfluidic channel, but close enough to heat source for react conditions to occur for about 30 seconds).

\subsection{Microfabrication}

Multiple microfabrication processes are necessary to create the main housing of my microfluidic reactor. The major processes include physical vapor deposition, photolithography, and wet/dry etching. These techniques generate channels on a silicon wafer. A glass wafer serves as the fourth wall of the microfluidic channel, which 
requires drilling of holes for inlets and outlets. Finally, anodic and plasma bonding steps serve to complete fabrication of the microfluidic reactor.

\subsubsection{Physical Vapor Deposition (Sputtering)}

In physical vapor deposition, the material to be deposited is vaporized, and the atoms in the vapor condense on a substrate [17]. There are several reasons for sputtering metals, such as aluminum, during microfabrication. The relevance to this project is that sputtered aluminum creates a mask layer which protects the silicon wafer during a later etching process.

Sputtering is a process that takes place inside a vacuum, while a controlled amount of argon gas is directed into the chamber. The gas plasma is struck with a radio-frequency (RF) power source, causing the gas to become ionized. The ions are accelerated toward the surface of the aluminum target because it is charged as a cathode. These ions knock off aluminum atoms creating a vapor. The atoms then condense on all exposed surfaces in the chamber, including the silicon substrate [18].

The thickness of an aluminum film is measured by placing an object (such as a microscope slide) over the edge of the substrate being sputtered. This creates a stepcoverage measurable using a profilometer. Since deposition rate varies with each cycle, users need to use this technique to determine the actual deposition rate. Identifying the thickness of aluminum is necessary in order to determine the etch time it will take to remove the aluminum mask in later steps. 


\subsubsection{Photolithography}

Photolithography is a process in which a mask design is imaged and developed into a light sensitive polymer coating on the surface of a silicon wafer (Figure 10). First, a spin coater applies an even coat of photoresist onto the surface of the wafer. There are two types of photoresist, negative and positive. This project uses positive photoresist, which is a polymer matrix that has the unique property of becoming soluble when exposed to UV light. The areas to be dissolved are where the channels will be etched into the silicon wafer.

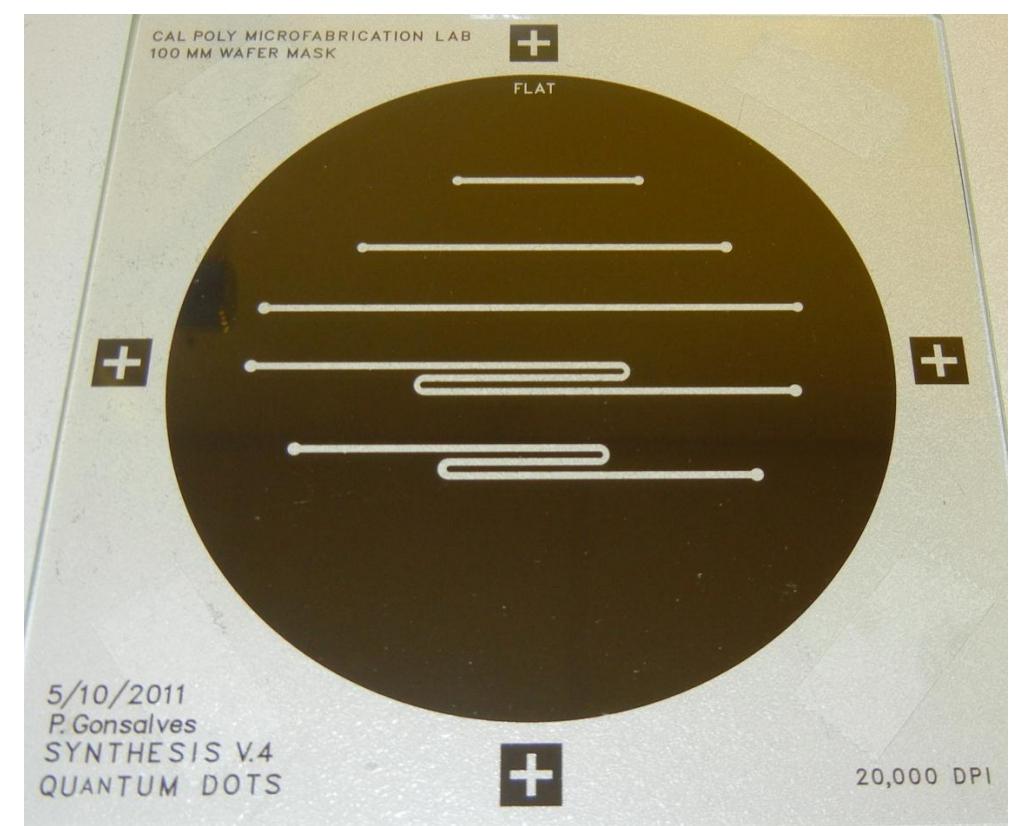

Figure 10 - The mask was designed to only allow light to expose specific areas of the wafer, namely $2.5,5,7.5,10$ and $12.5 \mathrm{~cm}$ channels.

The exposure dose (light integral setting) is based on the thickness of the photo resist and power density of the Hg-arc lamp. The Hg-arc lamp used in this project has a power density of $6.0 \mathrm{~mJ} / \mathrm{cm}^{2}$ (measured using an ILT1400A radiometer unit). The radiometer sensor is calibrated to respond to wavelengths between 350 and $450 \mathrm{~nm}$, which matches the response (absorption profile) of the Shipley 1813 Microposit positive resist 
[19]. Each light integral unit corresponds to 3.7122 seconds of exposure time (Figure 11).

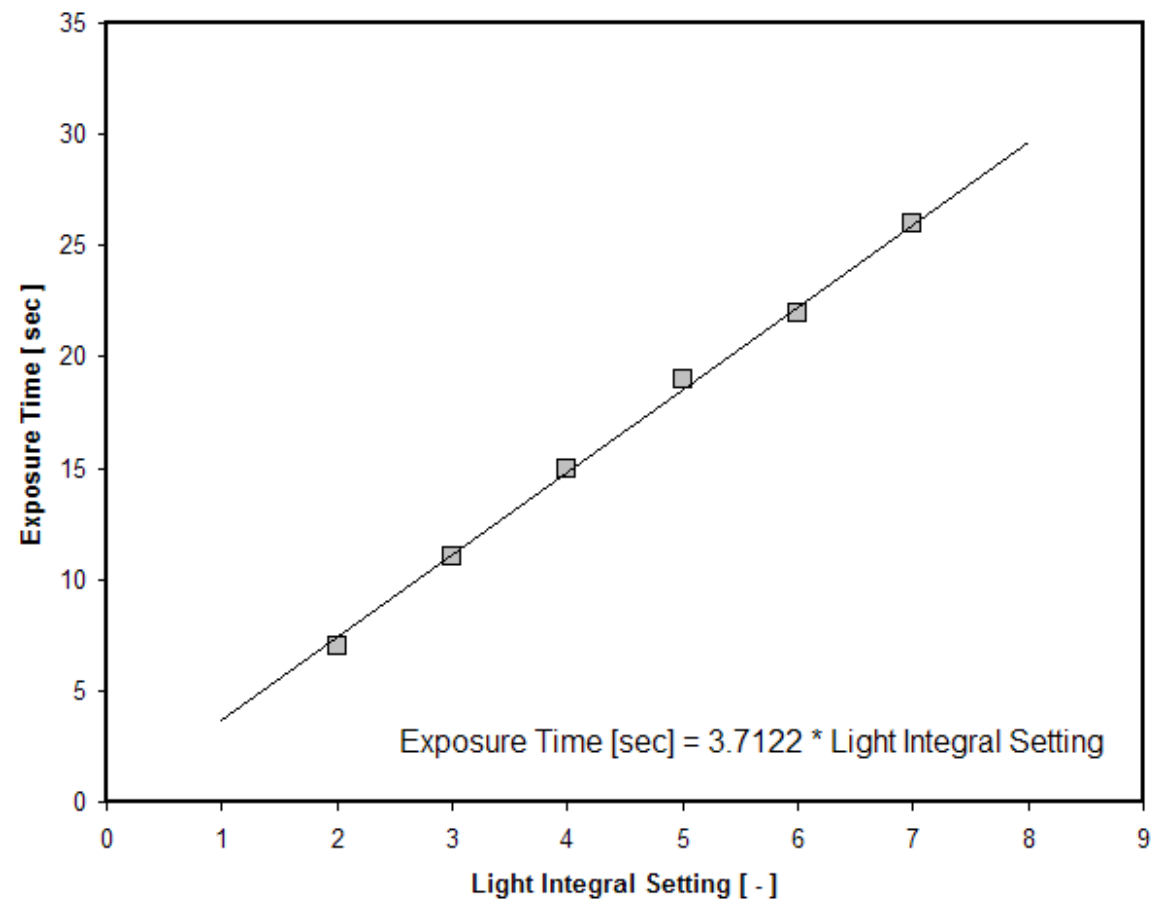

Figure 11 - Plot showing the linear relationship of exposure time to light integral setting.

\subsubsection{Wet Etching}

Etching is the process of selectively removing areas of the film on the substrate. There are two general types of etching: wet (chemicals) and dry (plasmas). This project uses both types of etching. Etch direction is an important aspect to consider in etching. Wet etching is typically isotropic, meaning it etches in all directions simultaneously. Isotropic wet etching leads to undercutting the mask layer. Dry etching is anisotropic, meaning that it prefers to etch mostly in one crystallographic direction. Anisotropic etching minimizes the undercutting of the mask and produces nearly vertical side walls [20]. 
In wet etching, the entire wafer is immersed in an acid bath (for metals, but silicon is immersed in TMAH base) and the reaction takes place at the liquid-solid interface. An acid is chosen that has a high selectivity ratio, which is the ratio of etch rates between two films in the same acid bath [20]. For example, after the developing step of photolithography, the unexposed photo resist remaining on the wafer becomes the mask layer. The aluminum film is uncovered in areas that were developed (Figure 12).

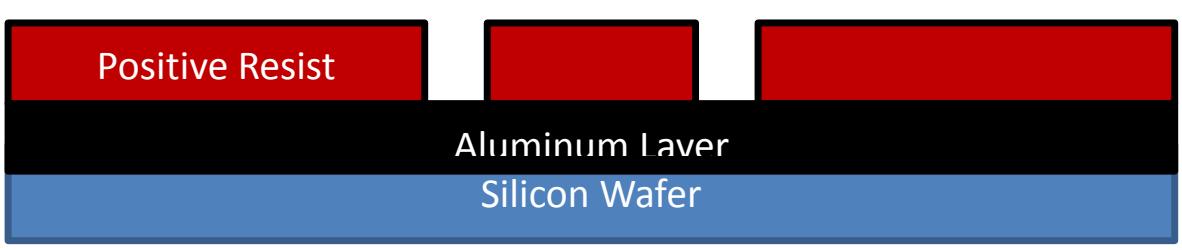

Figure 12 - Cross section showing that the developing solution will etch the channel design into the positive resist in areas that were exposed to UV light.

By immersing the wafer in an aluminum etchant (an acetic, nitric, and phosphoric acid mix at $50^{\circ} \mathrm{C}$ ), the exposed aluminum film is removed, exposing the original silicon substrate, while the photo resist remains. Aluminum etchant has high selectivity to etch aluminum over photo resist. Once etched, the photo resist is also removed by wet etching. Resist stripper has a selectivity that prefers to etch positive photo resist over aluminum or silicon. The result of these wet etching processes is the original mask image (channels) on the silicon wafer, with aluminum remaining as a mask.

\subsubsection{Reactive Ion Etching (Dry Etching)}

Reactive lon Etching (RIE) is a dry etching process. The process is considered dry because reactive gasses are used to etch, rather than acids. The wafer is placed in a plasma-enriched vacuum environment and the reaction takes place at the gas-solid interface [21]. Dry etching techniques are used to etch silicon because fluorine is highly 
reactive with silicon; thus, $\mathrm{SF}_{6}$ gas is an ideal choice as an etchant. The wafer is set on an electrode and given a negative bias, which accelerates positively charged fluorine ions toward the surface (Figure 13). The fluorine ions react with the silicon surface and create volatile $\mathrm{SiF}_{4}$ gas products that are vented into the atmosphere [22].

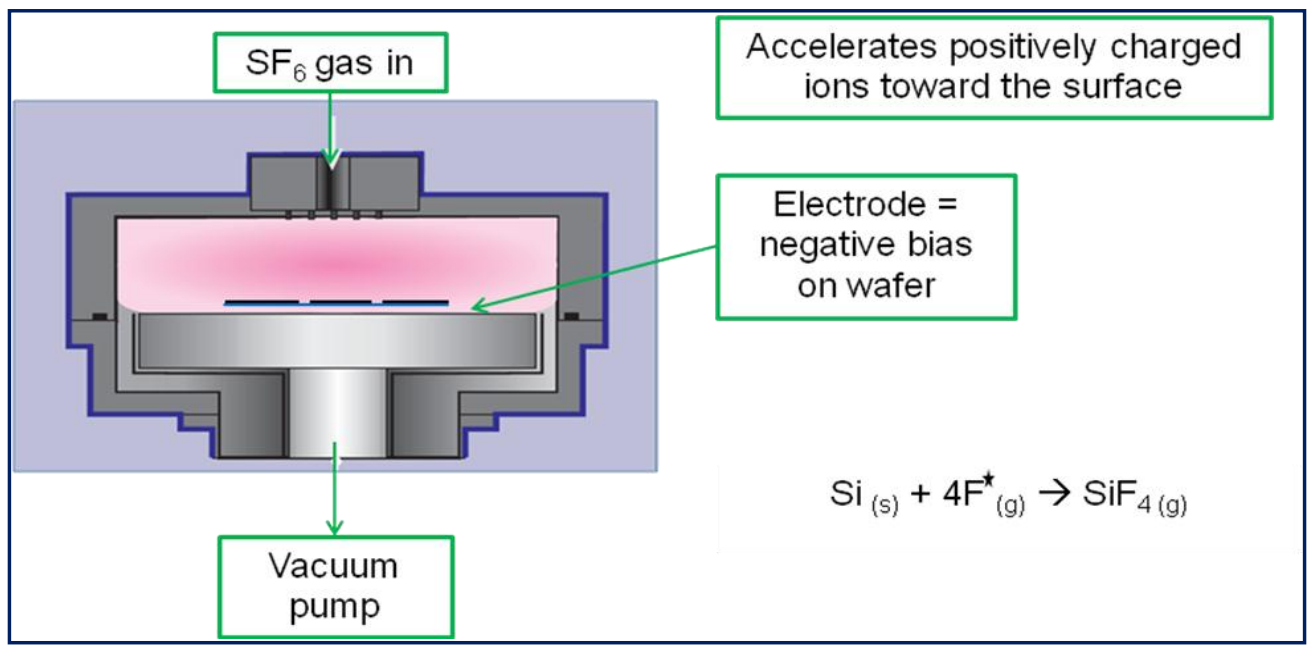

Figure 13 - Reactive lon Etching is an anisotropic dry-etching process whereby fluorine radicals aggressively react with the exposed silicon wafer

The RIE cycle results in the selective removal of silicon because RIE is highly selective to etching silicon over aluminum. Further, since RIE is an anisotropic etching process, the final product is channels with relatively vertical side walls. The final etching process is another wet etch to remove the aluminum mask layer.

\subsection{Anodic Bonding}

Anodic bonding is a wafer-bonding procedure without an intermediate layer. The process is done in four steps [23]:

1) Contacting the substrates

2) Heating up the substrates 
3) Bonding by applying an electrostatic field

4) Cooling down the wafer stack

A drawback to anodic bonding occurs when there is a difference in the coefficients of thermal expansions (CTE) between the glass and silicon substrates. Mismatch harms the bond through intrinsic material tensions within the used materials and causes disruptions in the bonding materials, i.e. the glass will shatter during bonding. The CTE of ordinary silica glass $\left(\mathrm{SiO}_{2}\right)$ is approximately $6.5 \mathrm{e}^{-7} \mathrm{strain} /{ }^{\circ} \mathrm{C}$, whereas silicon is $2.7 \mathrm{e}^{-6}$ strain $/{ }^{\circ} \mathrm{C}$. The use of sodium-containing glasses, such as Pyrex ${ }^{\circledR}$, supports to prevent CTE mismatch because the CTE of Pyrex ${ }^{\circledR}$ is $3.6 \mathrm{e}^{-6}$ strain $/{ }^{\circ} \mathrm{C}$, similar to silicon [24]. The composition of Pyrex ${ }^{\circledR}$ is shown in Table I.

Table I - Composition of Pyrex ${ }^{\circledR}$ Borosilicate

\begin{tabular}{|c|c|}
\hline Compound & \% Composition \\
\hline $\mathrm{SiO}_{2}$ & 81 \\
\hline $\mathrm{B}_{2} \mathrm{O}_{3}$ & 13 \\
\hline $\mathrm{Na}_{2} \mathrm{O}$ & 4 \\
\hline $\mathrm{Al}_{2} \mathrm{O}_{3}$ & 2 \\
\hline
\end{tabular}

It is necessary to ensure that both the silicon and Pyrex ${ }^{\circledR}$ substrates are free of contaminations prior to bonding; thus, both are run through the Spin-Rinse-Dryer before proceeding. The silicon and Pyrex ${ }^{\circledR}$ substrates are then stacked between two aluminum blocks, all of which are resting on a hot plate. The block above the glass is negatively charged to become a cathode, while the block below the silicon is positively charged to 
become an anode (Figure 14).

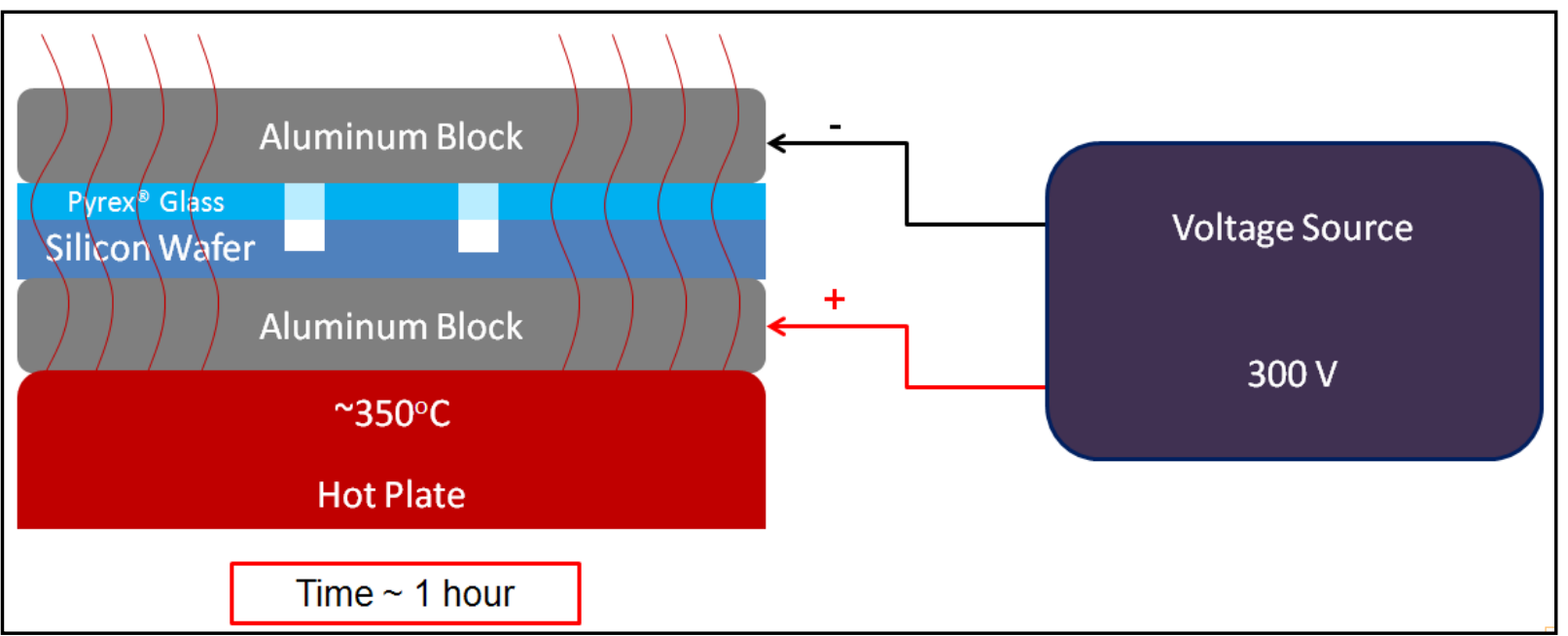

Figure 14 - Cross section showing the anodic bonding testing apparatus. The hot plate allowed for ion diffusion in the solid substrates, while the voltage was the driving force for anodic bonding to occur.

An electrostatic field is created when several hundred volts are applied to the stack, resulting in a diffusion of $\mathrm{Na}^{+}$ions out of the bond interface to the top side of the glass by the cathode. The remaining oxygen ions $\left(\mathrm{O}^{-}\right)$near the bond interface diffuse toward the anode and react to form $\mathrm{SiO}_{2}$ (Figure 15).

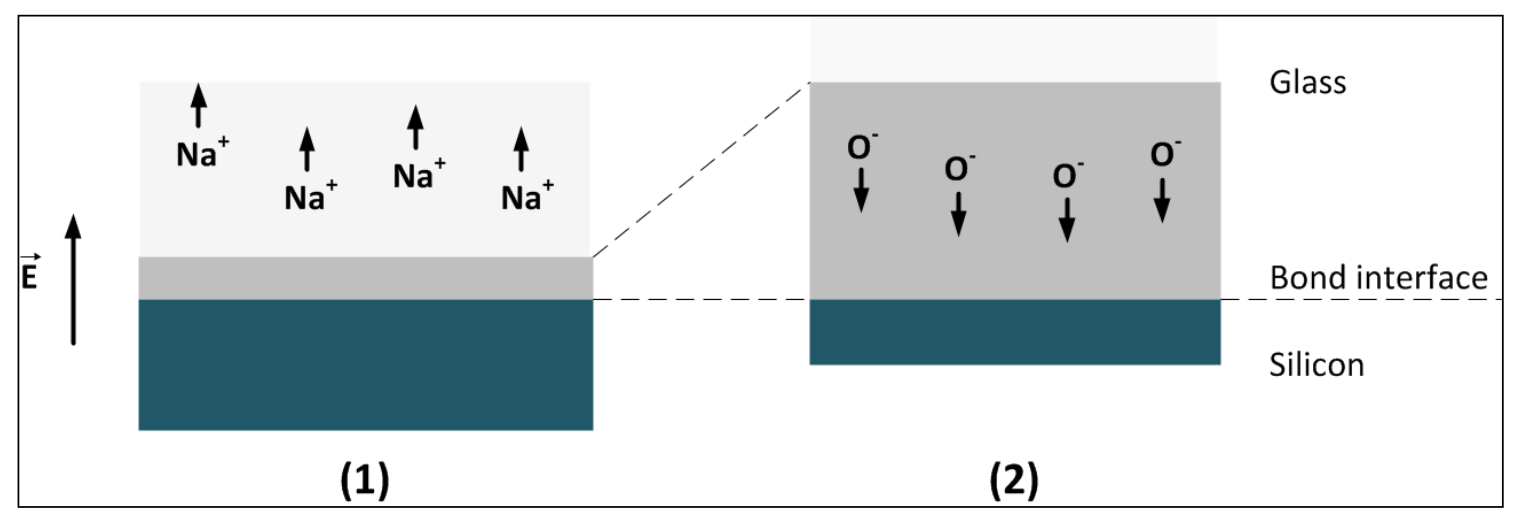

Figure 15 - lons drifting in glass during bonding are influenced by electrostatic field [25].

1) Formation of depletion zone (gray) through $\mathrm{Na}^{+}$drifting. 2) Drift of $\mathrm{O}^{-}$ions in the depletion zone. 
Bonding conditions increase with increased temperature (typically greater than $350^{\circ} \mathrm{C}$ ), greater voltage (typically greater than $300 \mathrm{~V}$ ), and longer time. The amount of time it will take to bond depends on the thickness of the materials, as well as the temperature and voltage values. The lower the temperature and voltage, the longer it will take to bond [26]. Once the bond is given sufficient time to complete, the voltage and temperature sources are turned off and the bonded wafer is allowed to cool. In this project, $300 \mathrm{~V}$ for 90 minutes provided sufficient time to allow anodic bonding to come to completion.

\subsection{Poly (dimethyl siloxane)}

Poly (dimethyl siloxane) (PDMS) belongs to a group of polymeric organosilicon compounds that are commonly referred to as silicones [27]. PDMS is comprised of repeat units of silicon and oxygen, with methyl groups attached to the silicon atoms (Figure 16).

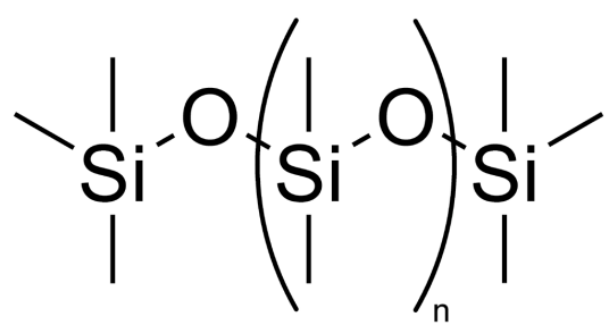

Figure 16 - Repeat structure of poly (dimethyl siloxane)

\subsubsection{Synthesis}

Poly (dimethyl siloxane) is transported in two components to remain in liquid form. When the polymer is ready for application, the base and curing agent are combined, typically in a 10 to 1 ratio. The curing time is greater than two hours; thus, significant 
time is available to degas the solution in a vacuum prior to curing [28]. PDMS is cured in an oven at $70^{\circ} \mathrm{C}$, whereby the solution will transform into a flexible elastomeric material.

\subsubsection{Argon Plasma Bonding}

Another reason that PDMS is a preferred material for microfluidics is because it readily bonds to itself and glass by using a simple plasma treatment. Plasma treatments alter the chain structure of PDMS, making it suitable for bonding. In plasma treatments, hydrogen atoms are first removed from the polymer chain to generate radicals within polymer chains located at the surface. Some of these radicals in the polymer chain combine themselves with the radicals formed in the plasma resulting in functional groups [29]. Basically, what occurs is the removal of the $\mathrm{CH}_{3}$ (methyl) groups, enabling Si-O-Si bonds to form between the surface of the PDMS and the surface of the glass.

The argon plasma cannot, by itself, introduce groups at the surface; however, the presence of oxygen and moisture in the air can form $\mathrm{SiO}_{2}, \mathrm{Si}-\mathrm{OH}$, or $\mathrm{Si}-\mathrm{CH}_{2} \mathrm{OH}$ groups on the PDMS surface [30]. Bonding occurs due to the condensation reaction between silanol groups, when plasma treated surfaces are pressed together (Figure 17).
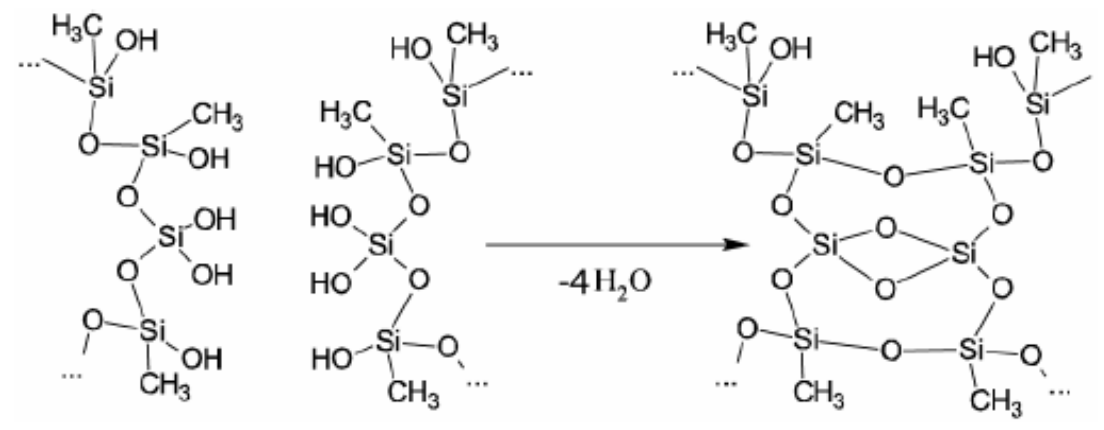

Figure 17 - Covalent bonding between plasma treated PDMS surfaces (as a result of condensation reaction between silanol groups) [31]. 
After plasma treatment, the PDMS and glass surfaces are mated immediately and a mild pressure is applied, followed by a heat treatment. A typical heat treatment is about 10 minutes at $70^{\circ} \mathrm{C}$ [32]. While in the oven, the PDMS and glass interfaces finish bonding and are ready for microfluidic application.

\subsection{Broader Impacts}

Multiple fields benefit from the use of quantum dots, such as photovoltaics, light-emitting diodes (LEDs), and biology. These three fields have the greatest demand for quantum dots and offer the most promising short-term benefits to society. However, careful considerations must be made to identify the pros and cons of quantum dot synthesis in areas such as manufacturability, environmental impact, economic impact, sustainability, ethical considerations, health and safety.

\subsubsection{Benefits to Science and Engineering}

In the field of photovoltaics, quantum dots increase the efficiency and thereby reduce the cost of the typical silicon photovoltaic cell. A layer of quantum dots applied to a solar panel can convert otherwise unused UV light from the sun into visible light that can make electricity [33]. Quantum dots made of lead selenide have been shown experimentally to produce as many as seven excitons from one high energy photon of sunlight (7.8 times the band gap energy) [34]. Conversely, today's photovoltaic cells manage only one exciton per high-energy photon, with high kinetic energy carriers losing their energy as heat. Theoretically, solar cell efficiency could increase between 
$31 \%$ and $42 \%$ with quantum dots. An additional advantage with quantum dot photovoltaics is that they enable thin film solar panel manufacturing companies to add a coating to the surface of the panel, which currently technology does not allow them the advantage, such as anti-reflective or self-cleaning coatings [35].

In recent years, there have been several promising inquiries into using quantum dots for LEDs to make displays and other solid state lighting sources. Quantum dots are valued for displays because they emit light that more accurately renders colors perceived by the human eye. Additionally, quantum dots require very little power since they are not color filtered. Displays that intrinsically produce monochromatic light are more efficient, since more of the light produced reaches the eye [7].

Finally, in modern biological analysis, a variety of organic dyes are typically used; however, there has been increased demand in the flexibility of these dyes [36]. Quantum dots fill the role because they are superior to organic dyes on several counts. Quantum dots are considerably brighter (owing to a high extinction coefficient combined with a comparable quantum yield to fluorescent dyes [37]), as well as more stable. A typical use is to attach antibodies or small-molecule ligands onto quantum dots, which target specific proteins on cells. One case study shows that researchers were able to observe quantum dots in the lymph nodes of mice for more than 4 months [38].

An objective of this project is to create a functioning microfluidic reactor capable of synthesizing CdSe quantum dots with a discrete size distribution. Ideally, the quantum 
dots synthesized through the microfluidic reactor will have a FWHM that is equal to, or smaller than, the bulk synthesis or commercial scale FWHM.

\subsubsection{Manufacturability}

The creation of quantum dots requires multiple steps, each one requiring precise control over multiple variables. Recent research has shown that high quality, robust quantum dots can be created using bench-top techniques [9]. While it is important that these lowtech synthesis methods do not reduce the quality or reliability of the quantum dots produced, the focus of this project will be to develop a microfluidic reactor capable of reducing the complexity and "guess work" of quantum dot production while still producing high-quality reliable results.

\subsubsection{Environmental Effects}

The life cycle of quantum dots is related to the life cycle of the chemicals in their synthesis, which are carcinogenic and environmentally harmful. Scientists today are focusing on what will happen when society begins to dispose of consumer products that contain quantum dots. Current research is investigating how quantum dots travel through soil and water, and how the particles accumulate in plants and earthworms. As quantum dots are mass produced in commercial products, the transport of quantum dots and metal oxide nanoparticles in the environment is a key concern [39]. At Cal Poly, QD waste is disposed of through standard chemical waste disposal practices. 


\subsubsection{Economic Factors}

The complicated and specialized techniques required to make quantum dots are relatively expensive. A large majority of the cost comes from the solvents involved in making the quantum dot precursor solutions. These costs are the main barrier for those wishing to work with them, particularly at the university level. Commercially-made quantum dots range from $\$ 200$ - $\$ 800$ for $5 \mathrm{~mL}$ of solution, which is not a cost many companies or research facilities can sustain [6]. Similarly, non-toxic phosphor dots go for $\$ 70$ - $\$ 400$ per $\mathrm{mL}[40]$.

The quantum dot project began at Cal Poly to create a practical method of producing quantum dots for research in Cal Poly's Nanotechnology Lab [9]. Given the high cost of commercially produced quantum dots, the method for fabricating quantum dots on the bulk scale was developed.

\subsubsection{Sustainability}

This project designed a process for manufacturing a reusable microfluidic reactor, such that future students can create and use it repeatedly for synthesis of quantum dots. The microfluidic channels of the reactor (silicon anodically bonded to a Pyrex ${ }^{\circledR}$ glass wafer) are capable of being used for repeatable and reproducible quantum dot synthesis reactions. Some of the minor (and cheap) components, such as the Tygon® tubing and PDMS, need to be replaced between syntheses, but the overall microfluidic reactor is sustainable and a cost effective method for synthesizing CdSe quantum dots. 


\subsubsection{Ethical Considerations}

There are often unknown risks or unintended consequences to developing new technologies. The advantages to developing quantum dots are well known, but there is much that is still unknown about the risk to the human and natural environments. Consideration must be made to these areas of concern throughout all stages of development and disposal of quantum dots and their precursor solutions. My thesis project follows all standard lab procedures dealing with the chemicals involved in microfabrication, as well as quantum dot synthesis and disposal.

\subsubsection{Health and Safety Issues}

In this project, the quantum dots are made from heavy metals and toxic chemicals, namely cadmium and selenium, which pose serious risks to the health of the people handling them, as well as the environment. There are restrictions worldwide on the use of heavy metals in many household goods, which mean that most cadmium-based quantum dots are not usable in consumer-good applications [41].

ZnS coatings are being explored by other Cal Poly students in order to increase the intensity of the fluorescence of the quantum dots. However, the $\mathrm{ZnS}$ coating may react in water and create toxic hydrogen sulfide, in addition to being air and moisture sensitive [42].

One of the more serious issues with quantum dots is their potential in vivo toxicity when used in biomedical applications. CdSe nanoparticles are highly toxic to cultured cells 
under UV illumination. The energy of UV irradiation is close to that of the covalent chemical bond energy of CdSe nanoparticles. As a result, semiconductor particles can be dissolved, in a process known as photolysis, and release toxic cadmium ions into the culture medium. In the absence of UV irradiation, however, quantum dots with a stable polymer coating have been found to be essentially nontoxic [43]. That being written, little is actually known about the excretion process of quantum dots from living organisms, so careful examination must be made before quantum dot applications in tumor or vascular imaging can be approved for human clinical use. [44] 


\section{Chapter 2 Methods and Materials}

\subsection{Microfluidic Reactor Design Process}

In order to develop and optimize a process for synthesizing a microfluidic reactor, the following methodology was used (Figure 18):

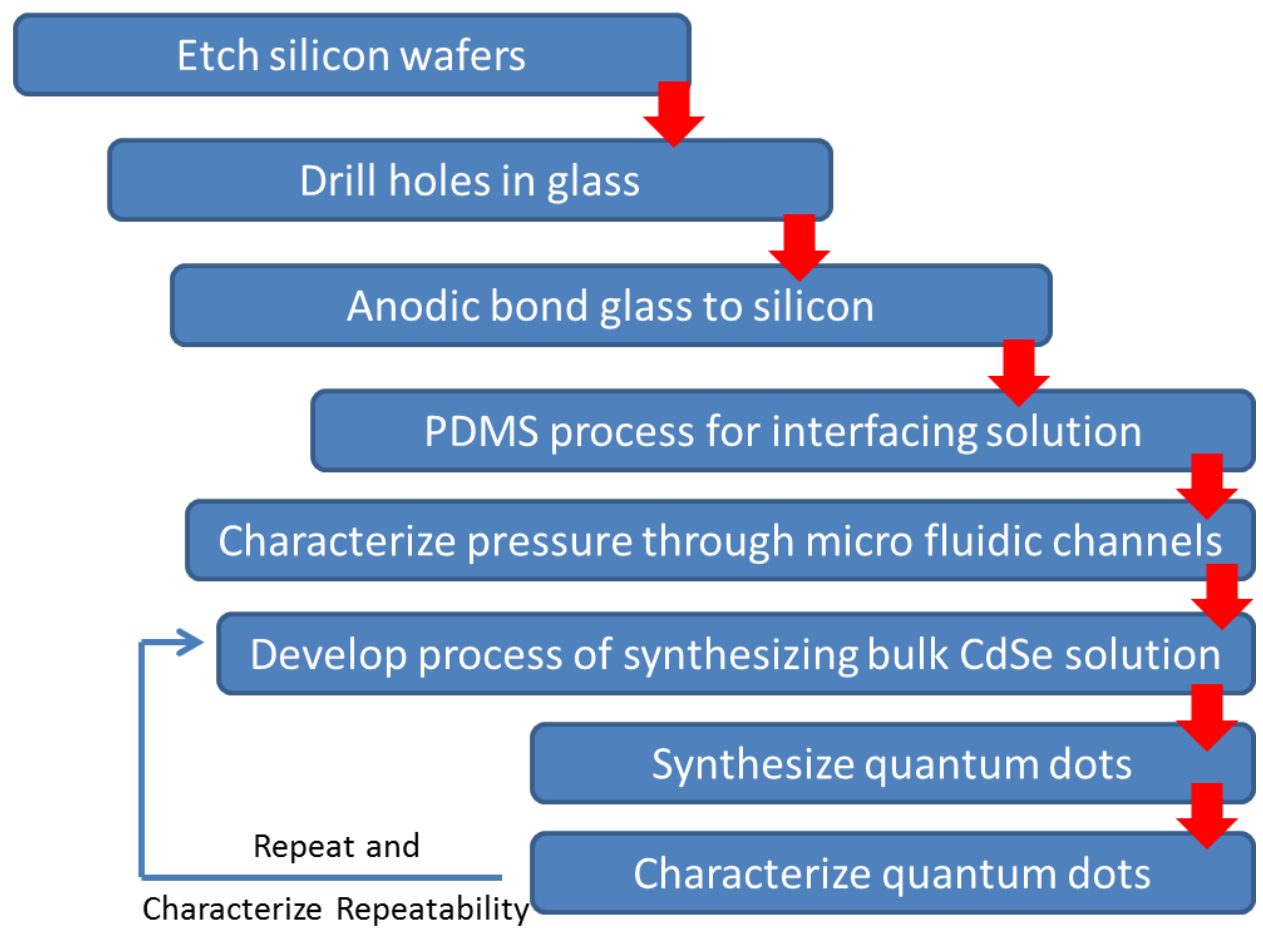

Figure 18 - Process development hierarchy. This scheme allowed for the micro fluidic reactor to be synthesized and characterized.

The manufacture of the microfluidic reactor was the culmination of many microfabrication steps. A silicon wafer was chosen as the bottom substrate of the reactor and was processed through physical vapor deposition (sputtering), photolithography, wet etching and reactive ion etching. Pyrex ${ }^{\circledR}$ was chosen as the top substrate and a process was developed to drill clean holes through the glass. An anodic bonding process was used to bond the two substrates together, while synthesis 
of PDMS was necessary to overcome problems with interfacing solutions from the bulk to the micro scale. Characterization of pressure in the microfluidic channels was necessary in order to avoid possible failures due to excessive pump rate or channels that were too long.

An unreacted CdSe solution was interfaced from a single syringe pump into the microfluidic reactor by using $\operatorname{Tygon}^{\circledR}$ and stainless steel tubing, in addition to plasma bonded PDMS. It was possible to synthesize QDs by controlling the CdSe flow rate and temperature by placing the microfluidic reactor on a hot plate set at $225^{\circ} \mathrm{C}$. CdSe QDs flowed out of reactor and were collected in a small vial for analysis. Finally, the CdSe QDs were characterized by exposing them to an excitation source (blue LED) and measuring the fluorescent spectral profile.

\subsection{QD Synthesis Design Process}

A syringe pump (Model NE-300) was used to ensure pump rate was under control during each test. The syringe pump accommodates a variety of syringe sizes. Pump rate is based on the inner diameter $(\mathrm{mm})$ of the syringe, which can be changed on the syringe pump's control panel prior to each test.

Bulk QD synthesis involves mixing the two precursor solutions at $225^{\circ} \mathrm{C}$, a temperature that was determined experimentally to be the ideal temperature to set the hot plate [9]. It is possible that the temperature within the microfluidic channels is lower than the hotplate temperature; however, the microfluidic reactor was not designed to 
accommodate a thermocouple at this time. Regardless, CdSe QDs have been shown to nucleate and grow at temperatures as low as $180^{\circ} \mathrm{C}$; therefore, $225^{\circ} \mathrm{C}$ on the hot plate was sufficient enough to synthesize QDs in the microfluidic reactor. For the sake of consistency, the same temperature was set on the hot plate for each QD synthesis; however, the temperature can become a variable in future tests as a means of characterizing the microfluidic reactor.

\subsubsection{Lab Setup}

Synthesis of cadmium and selenium precursors took place under a fume hood due to the toxic nature of the materials. Also, since octadecene makes up the majority of the CdSe solution, it was best to also carry out the microfluidic reaction procedures under a fume hood because octadecene fumes are an eye irritant.

The following pieces of lab equipment were used to create and operate the microfluidic reactor:

- Clean Air Products fume hoods (Model CAP1411-636-36H-PPHB \& SSHB)

- Torr CrC-150 Sputtering System with DCG-200 DC Plasma Generator

- Laurell Spin Coater (Model WS-400B-6NPP/LITE/AS)

- Canon Parallel Light Mask Aligner (Model PLA-501FA) with Ushio Mercury Lamp Power Supply (Model HB-25105AP)

- Semitool Spin/Rinse/Dryer (Model PSC-101)

- AGS RIE System (Model 1700-RIE) with ACG-6B RF Generator and Fluke 73III multimeter

- TriStar Technologies Duradyne Plasma Surface Treatment Station (Model PT200P)

- Ambios Technology Profilometer (Model XP-1)

- Hitachi 10" Bench Drill Press (Model B13F) 
- Quincy Lab Oven (Model 10)

- Ocean Optics Spectrometer (Model USB4000)

- GW Laboratory DC Power Supply (Model GPR-30H10D)

- Torrey Pines Scientific Hot Plate/Stirrer (Model HS50)

- Torrey Pines Scientific Hot Plate (Model H50)

- Barnstead|Thermolyne CIMAREC Hot Plate

- Thermoscientific CIMAREC Hot Plate

- Syringe Pump (Model NE-300)

- Pasco ${ }^{\circledR}$ PS 2107 Gas Pressure Sensor

- Pasco ${ }^{\circledR}$ Datalogger

\subsubsection{Reaction Procedure}

Under ideal conditions, the CdSe solution reacts by a nucleation and growth mechanism. CdSe clusters grow as long as they are allowed to react. The microfluidic reactor provides a suitable environment for this chemical reaction to take place. The reactor temperature was controlled by setting on a hot plate at $225^{\circ} \mathrm{C}$. The pump rate of the syringe pump ensured the reaction took place while moving through the microfluidic reactor channels and ended while the QDs were exiting through the outlet tubing of the device. In this project, a flow rate of $5 \mathrm{~mL} /$ hour was used, which translates to a residence time of approximately 1 second through the channel, but an additional 45 seconds of "void time" on the wafer, until the solution gets about 1 inch into the outlet tubing (more on this later).

\subsubsection{Design of Experiment (DOE)}

The main objective of this thesis project was to create a functioning microfluidic reactor. The success of the reactor was determined by its ability to withstand high pressures 
without failure, as well as synthesizing QDs within the microfluidic channels. As a result, this project had multiple DOEs. The primary DOE dealt with analyzing the QDs as they were synthesized through the microfluidic reactor channels; however, the secondary DOE dealt with interfacing a bulk solution into a microfluidic reactor using PDMS. A successful PDMS interface was necessary to overcome pressure problems that otherwise would have made QD synthesis impossible in the microfluidic reactor.

The microfluidic reactor was designed with multiple channel lengths; however, the DOE did not compare these variables. Rather, the DOE focused on the repeatability and reproducibility of QD synthesis by conducting reactions through the same length channel at the same pump rate. Synthesized QD solutions were characterized using fluorescence tests, which measured the wavelength FWHM, an indication of QD size distribution.

The second DOE focused on determining the ratio of PDMS base and curing agent that provided the greatest bonding strength to glass. For general use, the manufacturer recommends a mixing ratio of 10 (base) to 1 (curing agent). This ratio was tested against ratios of 5 to $1,7.5$ to 1,15 to 1 , and 20 to 1 , for a total of 5 different ratios.

Each ratio was prepared following the same PDMS mixing procedure [which follows in Section 2.2.7]. Further, the dimensions of each PDMS block were approximately equal (1" by 1 " by $1 / 4 ")$. Each PDMS chunk was bonded to Pyrex ${ }^{\circledR}$ using the Duradyne Plasma System. Using the Syringe Pump, a Pasco ${ }^{\circledR}$ Gas Pressure Sensor, and a 
Pasco ${ }^{\circledR}$ Datalogger, water was pumped into the $\operatorname{Tygon}^{\circledR}$ tubing that led to the PDMS/Glass interface until failure.

Measurements were made of the highest pressure achieved before failure of the PDMS/Glass bond. Failure pressure is directly proportional to the bonding strength of the PDMS to the Pyrex ${ }^{\circledR}$ glass.

In order to gain statistical significance of the results, each PDMS ratio was tested 4 times, for a total of 20 pressure tests. Additional tests may be necessary to eliminate outliers as a result of failures in other interfaces (SS316/Tygon ${ }^{\circledR}$ and SS316/PDMS). The results revealed a trend in bursting strengths and identified the mixture ratio that achieved the greatest pressures before failure. This ratio is the one used as the PDMS interface on the microfluidic reactor. The full procedure and results are available in Appendix A of this report.

\subsection{Microfabrication Processing Methods}

The first step to creating the microfluidic reactor was to secure a p-type silicon wafer with a $<100>$ crystallographic orientation. The wafer was cleaned of any dust or debris by immersing in a Piranha solution $\left(70^{\circ} \mathrm{C}\right)$ for 30 seconds, followed by a deionized (DI) water rinse. 


\subsubsection{Sputtering Aluminum}

Aluminum was determined to be the cheapest and most efficient mask to use in creating the microfluidic channels because of its selectivity in relation to silicon during reactive ion etching (typically $>30: 1$ ). The aluminum was sputtered using a Torr CRC-150 Sputtering System (Figure 19).

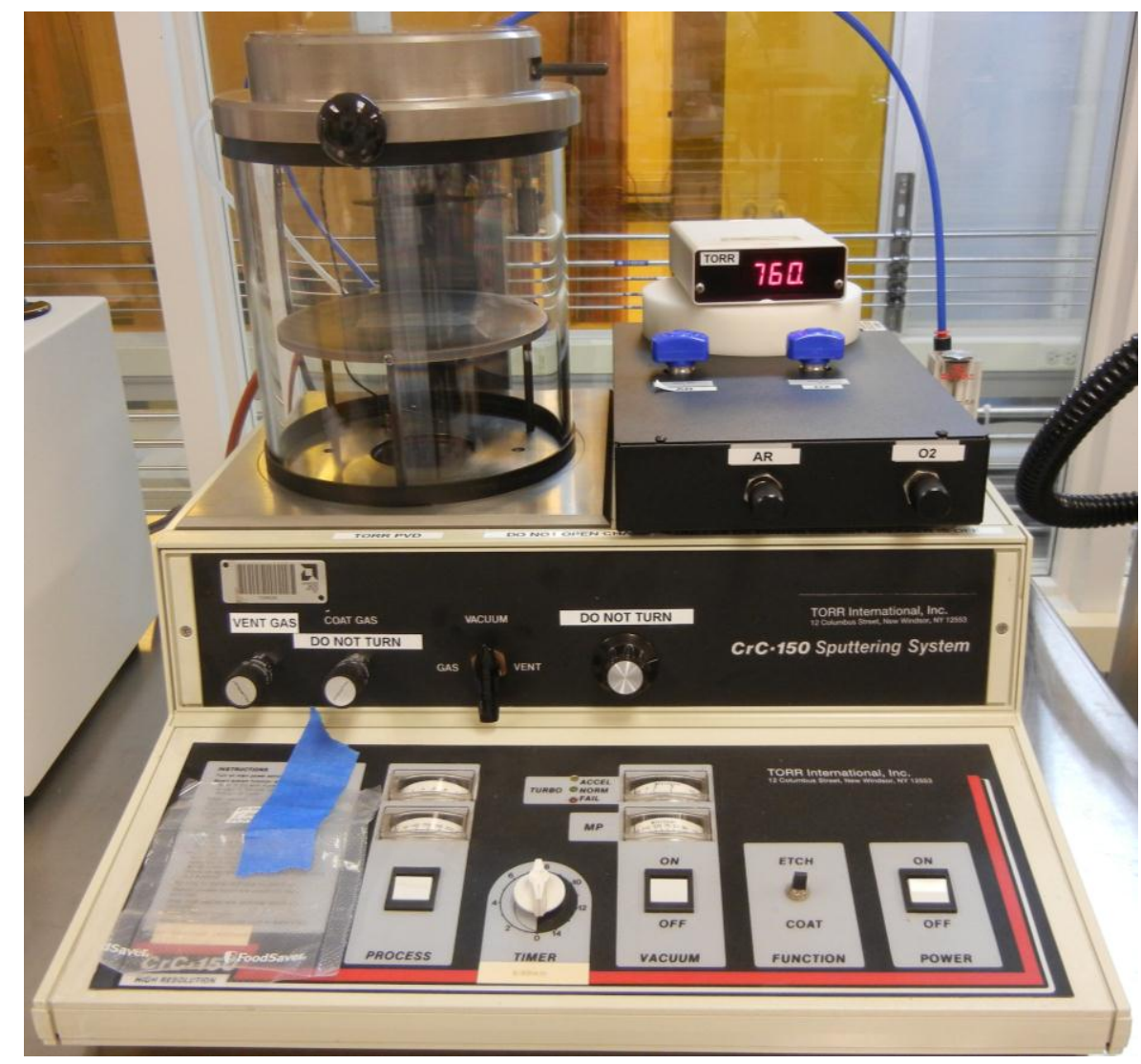

Figure 19 - The Torr CrC-150 Sputtering System

The following procedures were followed to sputter aluminum onto the silicon wafer:

1) The high purity argon and low purity nitrogen bottles and t-valves were opened.

2) The silicon wafer was placed in the vacuum chamber and surrounded by three glass microscope slides (to prevent wafer from sliding during pumping and venting).

3) The chamber gaskets were seated and coated with a thin layer of vacuum grease. 
4) The chamber was closed and the power switch was turned on.

5) The gas flow valve was turned to vacuum.

6) The vacuum switch was flipped to the "on" position

7) The function switch was set to 'coat'.

8) The system was brought to equilibrium at approximately $0.005 \mathrm{mTorr}$ (which took about 30 minutes).

9) The shutter was closed.

10) The gas flow valve was set to gas and the pressure was adjusted to 0.015 mTorr.

11) The following settings were entered on the Rohwedder PVD's main control panel:
a. Select Device (F3)
b. Select Master Gun Power ( $\uparrow \downarrow$ arrows)
c. Press F1 to turn On

12) The master circuit breaker on the ENI-DCG-200 was flipped to the ON position.

13) The output was set to 60 watts.

14) The on/off button on the top left of the front panel on the DCG-200 control panel was turned on to begin pre-sputter.

15) Two minutes was allowed for pre-sputter.

16) The shutter was then opened.

17) Fifteen minutes was allowed to pass for the physical vapor deposition of aluminum.

18) The shutter was closed.

19) The on/off button on the DCG-200 control panel was pressed to turn off.

20) The master circuit breaker on the DCG-200 was flipped to the OFF position. 
21) The gas flow valve was turned to the vacuum position.

22) The vacuum switch was turned to "off" and the pressure was brought to greater than 50mTorr before proceeding (allowing time for the Turbo Molecular Pump to slow down).

23) The gas flow valve was slowly turned to vent.

24) The power switch was flipped to "off".

25) The main power supply on the Rohwedder was turned OFF.

26) The high purity argon and low purity nitrogen gas bottles and t-valves were closed.

The final result was an even layer of aluminum over the surface of the silicon wafer (Figure 20) using the parameters summarized in Table II.

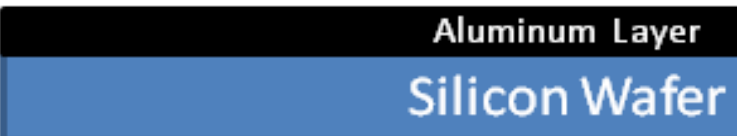

Figure 20 - Cross section of silicon wafer with a layer of aluminum deposited on top

Table II - Parameters for Sputtering Aluminum on Silicon Wafer

\begin{tabular}{|c|c|c|c|c|}
\hline $\begin{array}{c}\text { Pressure, } \\
\text { mTorr }\end{array}$ & $\begin{array}{c}\text { Power, } \\
\text { Watts }\end{array}$ & $\begin{array}{c}\text { Pre-Sputter } \\
\text { time, min }\end{array}$ & $\begin{array}{c}\text { Sputter time, } \\
\text { min }\end{array}$ & $\begin{array}{c}\text { Sputter rate, } \\
\AA / \text { min }\end{array}$ \\
\hline 0.015 & 60 & 2 & 15 & 750 \\
\hline
\end{tabular}

During sputtering, Argon ions bombarded an aluminum target, knocking off aluminum atoms. The resultant aluminum vapor deposited aluminum atoms on the silicon substrate (Figure 21), which correlates to a thickness of $1125 \mathrm{~nm}$. 


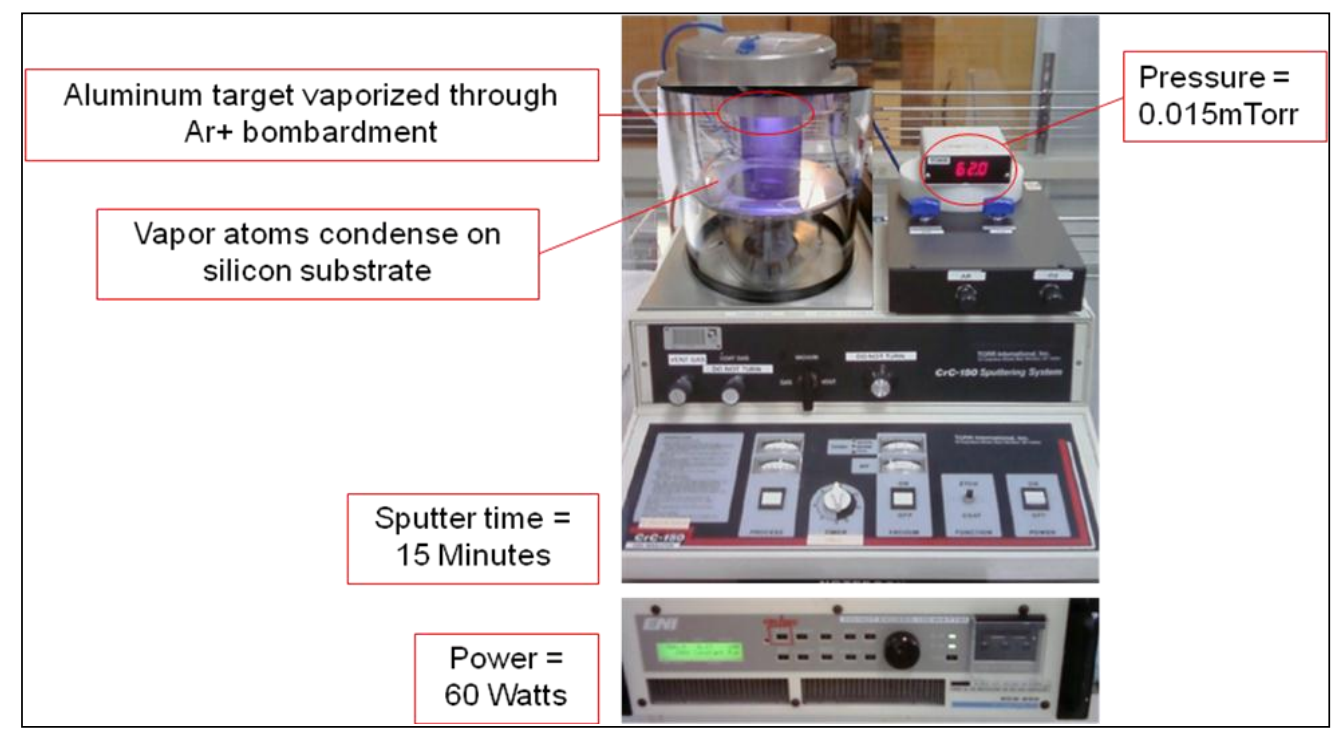

Figure 21 - The CRC-150 Sputtering System created an aluminum vapor fog that deposited aluminum atoms in an even layer on the silicon substrate.

\subsubsection{Photolithography}

The next processing step was to get the image of the channels onto the aluminum mask by performing photolithography on the wafer. Photolithography began by putting a coating of positive photo resist onto the wafer by using a Laurell Spin Coater (Figure 22).

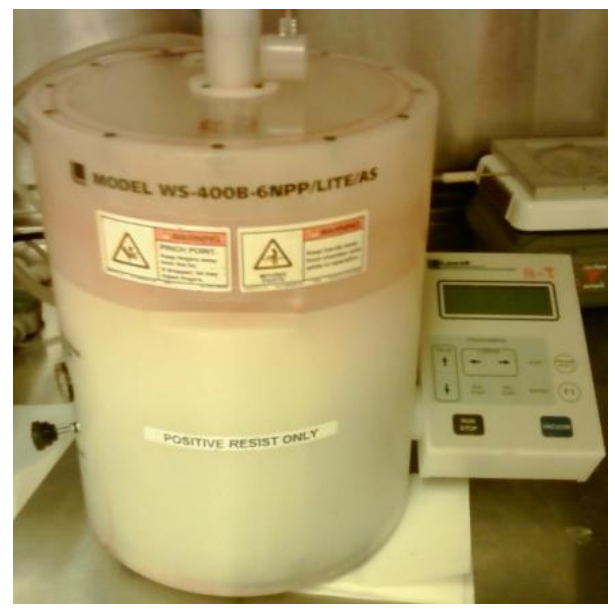

Figure 22 - The Laurell Spin Coater was used to evenly apply a layer of positive photo resist on top of the aluminum mask layer 
The following procedures were followed to spin coat positive photo resist:

1) Approximately $5 \mathrm{~mL}$ of Shipley 1813 (positive photo resist) was prepared in a syringe and allowed it to come to room temperature (overnight).

2) The vacuum pump in the Chase was turned on.

3) The low purity Nitrogen gas bottle and t-valve were opened.

4) The main breaker switch on the top left corner of the fume hood was flipped on.

5) The two hot plates under the spin-coat fume hood were turned on and set to $90^{\circ} \mathrm{C}$ (soft bake) and $150^{\circ} \mathrm{C}$ (hard bake).

6) The thermocouple multimeter was turned on to monitor the temperatures of the two hot plates. Each hot plate was allowed to come to equilibrium.

7) The wafer was placed on the hard bake hot plate for approximately 5 minutes to evaporate any water that may have been on the wafer.

8) The wafer was then placed on the cold plate to chill for 30 seconds.

9) The spin coater was turned on by pressing the power switch on the back right of the spin coater housing.

10) The valves of the two hoses behind the spin coater were turned to the open position (vacuum hose and low purity nitrogen hose).

11) The wafer was loaded onto the spin chuck using the wafer centering tool and a vacuum was applied to the wafer by pressing the vacuum button on the control panel.

12) Approximately $5 \mathrm{~mL}$ of MicroChem Primer $80 / 20$ [containing $80 \%$ Hexamethyldisilazane (HDMS)] was dispensed onto the wafer and the lid was closed to the spin coater..

13) Program $A$ was selected on the spin coat process panel, which followed the process parameters listed in Table III. 
Table III - Process Parameters for Spin Coating

\begin{tabular}{|c|c|c|c|}
\hline Step & Purpose & Time, Sec & $\begin{array}{c}\text { Spin speed, } \\
\text { RPM }\end{array}$ \\
\hline 1 & $\begin{array}{c}\text { Post-Dispense } \\
\text { HMDS }\end{array}$ & 30 & 300 \\
\hline 2 & Spread HMDS & 20 & 3000 \\
\hline \multicolumn{4}{|c|}{${ }^{* *}$ Pause cycle and dispense 5mL of Positive Resist ${ }^{* *}$} \\
\hline 3 & Spread Resist & 20 & 200 \\
\hline 4 & Spread Resist & 10 & 500 \\
\hline 5 & Planarize & 20 & 4000 \\
\hline 6 & Resist & 5 & 300 \\
\hline
\end{tabular}

14) The run/stop button was pressed to start the spin coating cycle.

15) The entire spin-coating process was repeated a second time, excluding the dispensing of HMDS, to ensure complete coverage of photo resist.

16) Upon completion of the second spin coating cycle, the lid was opened and the vacuum button was pressed to release the wafer.

17) The wafer was placed on the soft bake hot plate for 60 seconds to drive off solvents.

18) The wafer was placed on a cold plate to chill for approximately 30 seconds.

19) Using acetone and wipes, the positive resist was cleaned out of the spin coat chamber, including the collection cup behind the spin coater.

20) The vacuum and nitrogen tubes behind the spin coater were closed.

21) The spin coater was powered off. 
The next step of photolithography was to run the wafer through the photolithography aligner to expose UV light through the mask onto the positive photo resist (Figure 23; Figure 24).

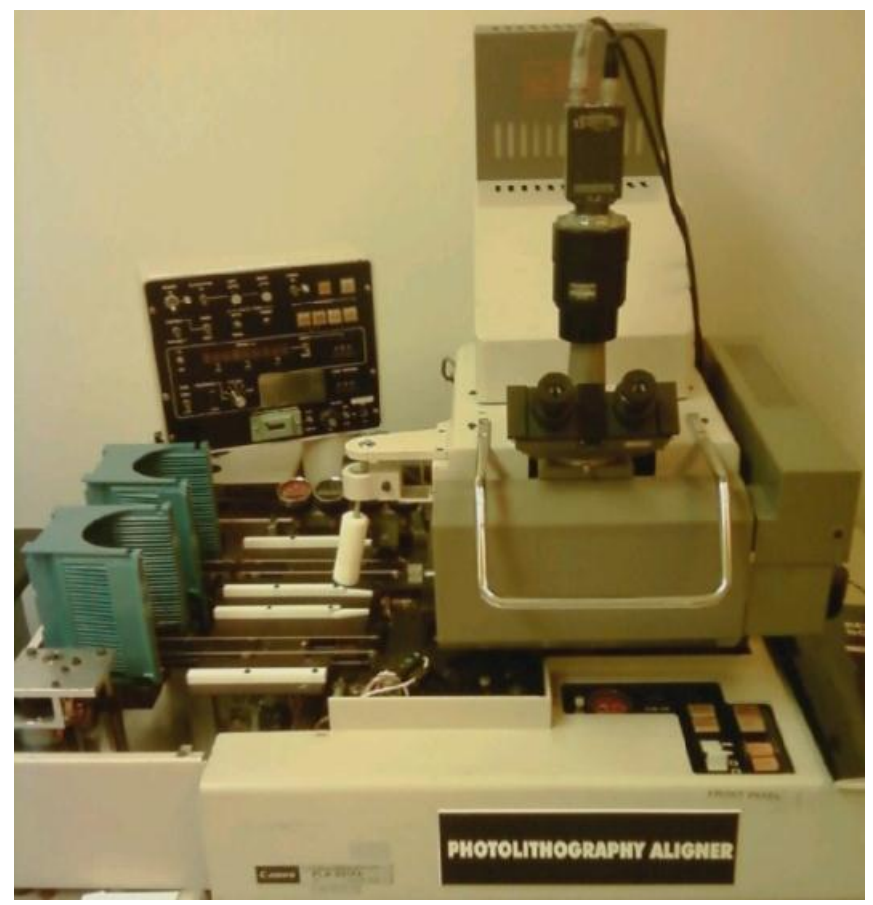

Figure 23 - The Photolithography Aligner allows the user to expose their device to UV light through a mask layer which has a desired pattern

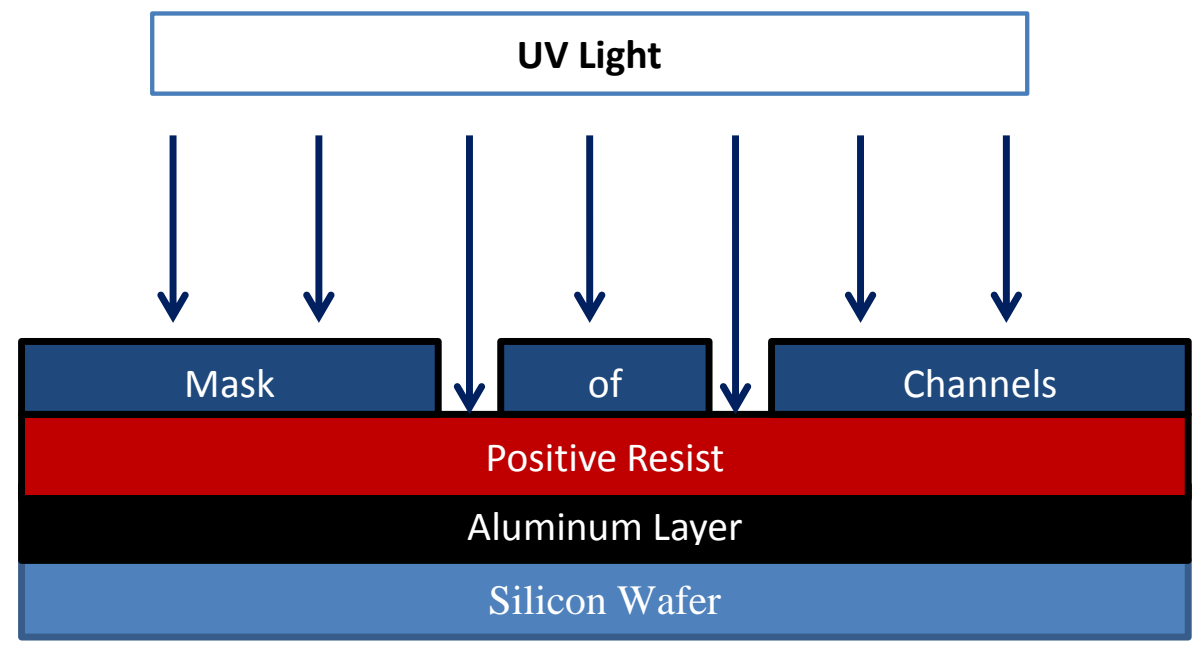

Figure 24 - Cross section showing that the positive photoresist will only be exposed to UV light in areas where the mask is has openings

The mask used had five different 1000-micron-wide channel lengths between $2.5 \mathrm{~cm}$ and $12.5 \mathrm{~cm}$ (Figure 25). 


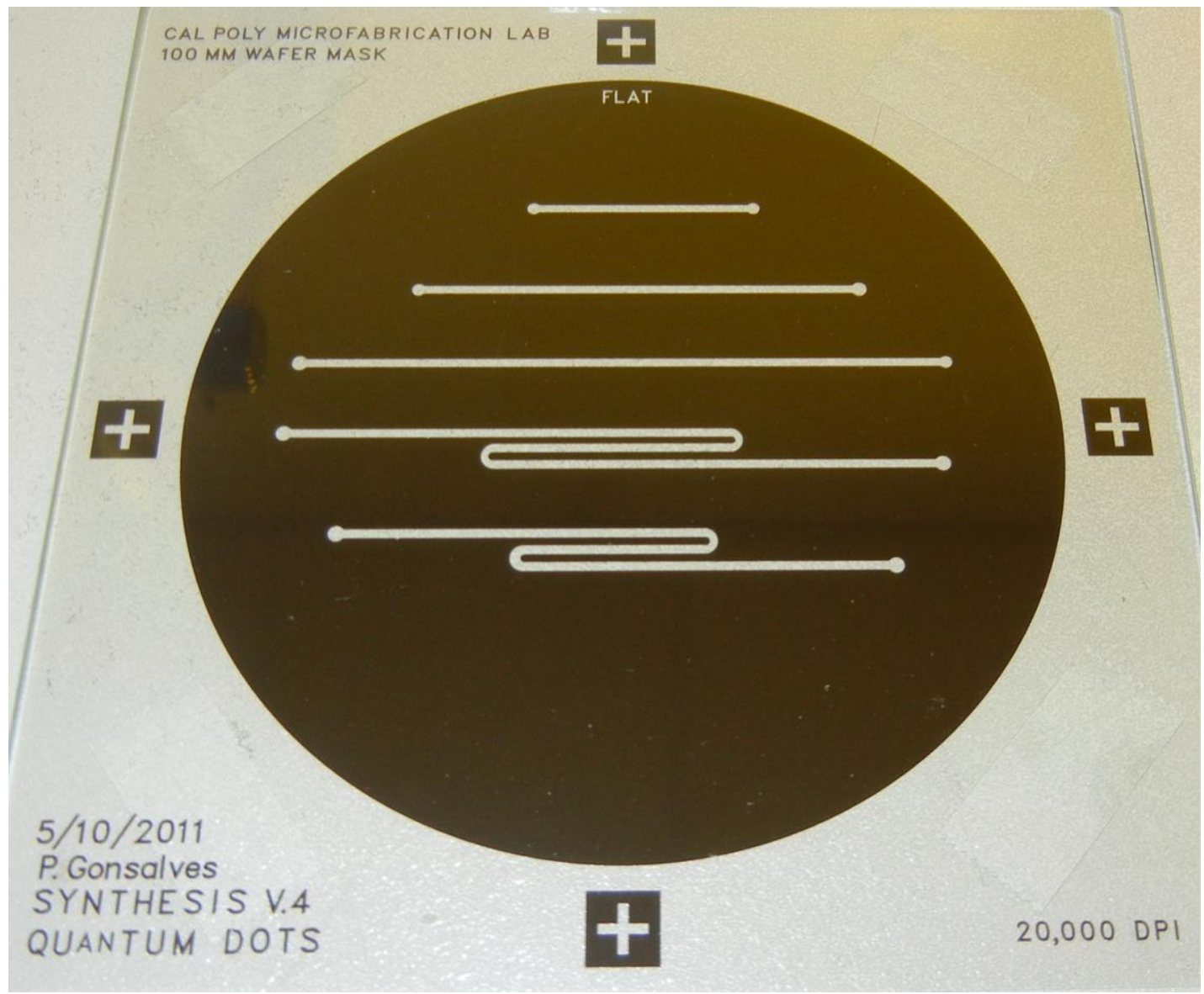

Figure 25 - The mask was designed to only allow light to expose specific areas of the wafer, namely 2.5, $5,7.5,10$ and $12.5 \mathrm{~cm}$ channels.

The following procedures were followed to use the photolithography aligner:

1) The Mercury Vapor Lamp was turned on and allowed 30 minutes to warm up.

2) The vacuum pump in the chase was turned on.

3) The low purity nitrogen bottle and t-valve in the chase were opened.

4) The valves behind the aligner for the vacuum, nitrogen and clean air hoses were opened.

5) The gauges on the aligner were confirmed to show:
a. Vacuum gauge $>60 \mathrm{cmHg}$
b. Clean air gauge $>2.5 \mathrm{~kg} / \mathrm{cm}^{2}$
c. Pressure gauge $>3.5 \mathrm{~kg} / \mathrm{cm}^{2}$
d. $\mathrm{N}_{2}$ gauge $>1.0 \mathrm{~kg} / \mathrm{cm}^{2}$ 
6) The main power switch located on the control panel was flipped to the ON position.

7) Typical settings on control panel were set:

a. Illuminator OFF

b. Laser OFF

c. Proximity selected

d. Wafer Feed set to AUTO

e. Alignment switch set to $1^{\text {st }}$ Mask

f. Alarm OFF

8) The mask was cleaned using DI water and filtered $\mathrm{N}_{2}$ air.

9) The OPTICS RELEASE LEVER was pulled and the optics was rotated $90^{\circ}$ counter-clockwise to access the mask holder plate.

10) The Mask Load button was pressed to release the glass plate already in the mask holder.

11) The mask was placed on the mask holder and aligned to the three pins (oriented so the words on the mask could be read).

12) The Mask Load button was pressed to vacuum seal the mask to the holder.

13) The optics were turned back to position over the mask.

14) The wafer was loaded into a blue cassette and put on the Load platform, while an empty blue cassette was placed on the Take-up platform.

15) The Light Integral to set to 4.0 .

16) The Front Panel was set:

a. Alignment Gap: $30 \mu \mathrm{m}$

b. Print Gap: $1.0 \mu \mathrm{m}$

17) The START button was pressed and the aligner cycled through UV exposure following the process parameters summarized in Table IV. 
Table IV - Process Parameters for Photo Alignment

\begin{tabular}{|c|c|c|c|c|}
\hline Dose, $\mathrm{mW} / \mathrm{cm}^{2}$ & $\begin{array}{c}\text { Alignment Gap, } \\
\mu \mathrm{m}\end{array}$ & Print Gap, $\mu \mathrm{m}$ & Light Integral & $\begin{array}{c}\text { Exposure } \\
\text { Time, sec }\end{array}$ \\
\hline 6.5 & 30 & 10 & 4.0 & 14.85 \\
\hline
\end{tabular}

18) Immediately after exposure, the wafer was developed in Microposit CD-26

Developer [2.5\% Tetramethylammonium hydroxide (TMAH)] using the parameters summarized in Table V.

Table V - Process Parameters for Developing Exposed Positive Photoresist

\begin{tabular}{|c|c|c|}
\hline Time, minutes & Temperature, ${ }^{\circ} \mathrm{C}$ & Agitation? $(\mathrm{Y} / \mathrm{N})$ \\
\hline 2 & Room Temp & Yes \\
\hline
\end{tabular}

19) The wafer was placed on the hard bake hot plate for 60 seconds, followed by 30 seconds on a cold plate to chill (Figure 26).

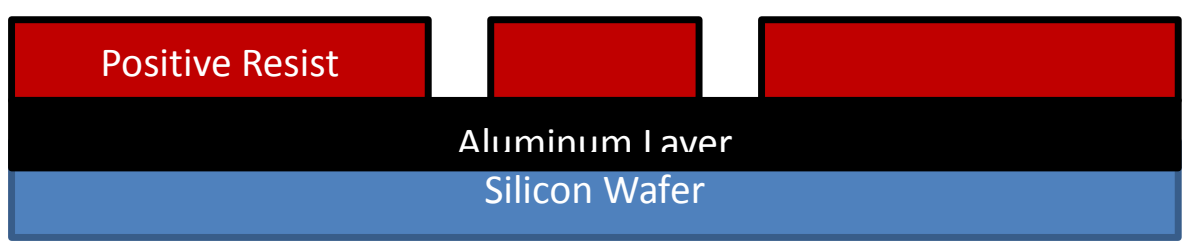

Figure 26 - Cross section showing that the developing solution will etch the channel design into the positive resist in areas that were exposed to UV light.

After development, the aligner was shut down, the chemical waste was disposed of in the appropriate waste containers and the air and vacuum systems were closed and turned off.

\subsubsection{Wet Etching and Resist Strip}

The next processing step was to etch the channel design into the now-exposed aluminum mask (Figure 27). 


\section{Positive Resist}

\section{Aluminum Layer}

\section{Silicon Wafer}

Figure 27 - Cross section showing that aluminum etchant will etch the channels into the areas of aluminum that were exposed to the etchant

Wet etching was done by the following these procedures:

1) $500 \mathrm{~mL}$ of aluminum etchant [Acetic acid, Nitric acid, Phosphoric acids;

Transene: Type A] was poured into a large flask.

2) The flask was set on a hot plate, under a fume hood, and brought to equilibrium at $50^{\circ} \mathrm{C}$. The temperature was monitored with a glass thermometer.

3) Using a Teflon wafer cassette, the wafer was immersed in the etchant for about 2 minutes, with occasional agitation every $20-30$ seconds.

4) The Teflon cassette was removed from the solution and rinsed by dunking in a $2000 \mathrm{~mL}$ flask of deionized (DI) water 2-3 times.

5) The wafer was run through a Spin-Rinse-Dry cycle.

A summary of the wet etch parameters is shown in Table VI.

Table VI - Process Parameters for Aluminum Etching

\begin{tabular}{|c|c|c|c|}
\hline Time, minutes & Temperature, ${ }^{\circ} \mathrm{C}$ & Etch Rate, $\AA / s e c$ & Agitation? (Y/N) \\
\hline 2 & 50 & 750 & Yes \\
\hline
\end{tabular}

6) Approximately $500 \mathrm{~mL}$ of Microposit Remover 1165 [94-95\% 1-methyl-2pyrrolidinone; 5-6\% Pyrrolidone Compound] was poured into another flask.

7) The flask was set on a hot plate, under the fume hood, and brought to equilibrium at $70^{\circ} \mathrm{C}$. The temperature was monitored with a glass thermometer.

8) Using a Teflon wafer cassette, the wafer was immersed in the Microposit Remover for 15 minutes, while agitating every 2-3 minutes.

9) Once the resist was completely removed, the Teflon cassette was removed from the solution and dunked in the flask of DI water 2-3 times. 
10) The wafer was run through another Spin-Rinse-Dry cycle.

The resulting silicon wafer now only had an etched aluminum film (Figure 28).

Aluminum Layer

\section{Silicon Wafer}

Figure 28 - Cross section showing the silicon wafer with an aluminum mask layer protecting areas that were not to be etched.

A summary of the parameters for stripping off the positive resist are shown in Table VII.

Table VII - Process Parameters for Stripping Positive Resist

\begin{tabular}{|c|c|c|}
\hline Time, minutes & Temperature,$~^{\circ} \mathrm{C}$ & Agitation? $(\mathrm{Y} / \mathrm{N})$ \\
\hline 15 & 70 & Yes \\
\hline
\end{tabular}

\subsubsection{Reactive Ion Etching}

The next processing step was to create channels in the silicon wafer by using reactive ion etching (RIE). This was done by the following these procedures:

1) The low-purity nitrogen, high-purity oxygen, and SF6 gas bottles and t-valves in the chase were opened.

2) The lid to the RIE was opened and the wafer was placed in the center of the chamber. The wafer was secured by placing 3 glass microscope slides around/against the edges of the wafer.

3) The lid was closed and the MP Button was turned ON.

4) The RUF switch was flipped to "RUF ON" and a timer was started.

5) The BASE set point gage was set to 310 by manually turning the digits.

6) The vacuum was given time to pump down to $~ 50$ mTorr.

7) The GAS ONE switch was flipped to "MANUAL ON" (SF6) and the pressure was adjusted to 160 mTorr by using the Gas One Flow knob. The pressure was given ample time to reach equilibrium. 
8) The GAS TWO switch was flipped to "MANUAL ON" $\left(\mathrm{O}_{2}\right)$ and the pressure was adjusted to 200 mTorr by using the Gas Two Flow knob. The pressure was given ample time to reach equilibrium.

9) The Valve Control key was turned from the "Local Open" position to the "Remote" position to remotely adjust the base pressure of the chamber to 300 mTorr.

10) The FLUKE was turned on to monitor the voltage in the chamber.

11) The RF switch was flipped to "RF ON" and a timer was started.

12) The wattage was adjusted to 300 watts by turning the large black dial.

13) After 30 minutes, turn the RF switch was flipped to "RF OFF", the key remote was turned back to "Local Open", GAS ONE and GAS TWO were flipped to "MANUAL OFF", and the FLUKE was turned off.

14) The chamber was vented for 1 second by flipping to VENT ON, and then flipped back to VENT OFF. After 10 seconds, the vent process was repeated.

15) The RUF switch was flipped to "RUF OFF" and the vent switch was flipped to "VENT ON".

16) The MP switch was flipped to "MP OFF".

17) The lid was opened and the wafer was removed.

Since etch rate/depth varied with each test run the wafer was examined using a profilometer to determine the actual etch depth of the channels.

A summary of the parameters for RIE and final etch depth are shown in Table VIII.

Table VIII - Process Parameters for Reactive Ion Etching

\begin{tabular}{|c|c|c|c|c|}
\hline Ratio, $\mathrm{SF}_{6}: \mathrm{O}_{2}$ & $\begin{array}{c}\text { Base Pressure, } \\
\text { mTorr }\end{array}$ & Power, Watts & $\begin{array}{c}\text { Etch Time, } \\
\text { minutes }\end{array}$ & Etch depth, $\mu \mathrm{m}$ \\
\hline $80: 20$ & 300 & 300 & 30 & 15 \\
\hline
\end{tabular}

The RIE formed channels in the silicon wafer (Figure 29). 


\section{Aluminum Layer}

\section{Silicon Wafer}

Figure 29 - Cross section showing the etched channels that result from Reactive lon Etching

If additional etching was necessary, the wafer was placed back in the RIE chamber and the process was repeated until the desired depth was achieved. Once the desired etch depth was achieved, the aluminum mask was stripped off using the aluminum etchant and the process parameters in Table VI. The final result was an etched silicon substrate ready for anodic bonding (Figure 30).

\section{Silicon Wafer}

Figure 30 - Cross section showing etched silicon wafer after stripping off the aluminum mask

\subsubsection{Drilling Holes in Pyrex ${ }^{\circledR}$}

The Pyrex ${ }^{\circledR}$ wafer was aligned over the original mask to identify the location of where the inlet and outlet holes were to be drilled (Figure 31).

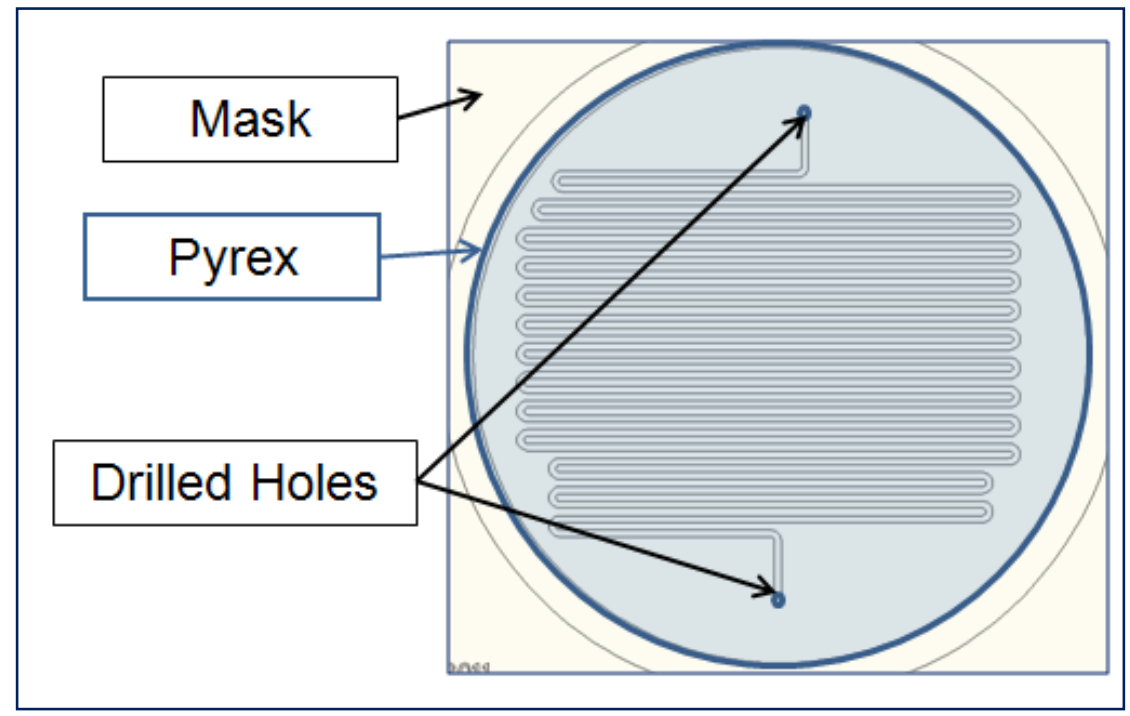

Figure 31 - The Pyrex ${ }^{\circledR}$ wafer is lined up over the mask layer and marked to identify where the inlet and outlet holes will go 
Drilling through glass creates small "chips" around the "breakthrough" side of the hole, causing problems during anodic bonding. Since the chipping was virtually non-existent on the side of the glass wafer that drill bit contact was initiated, it was imperative that the holes were drilled from the "anodic bonding side" (Figure 32).

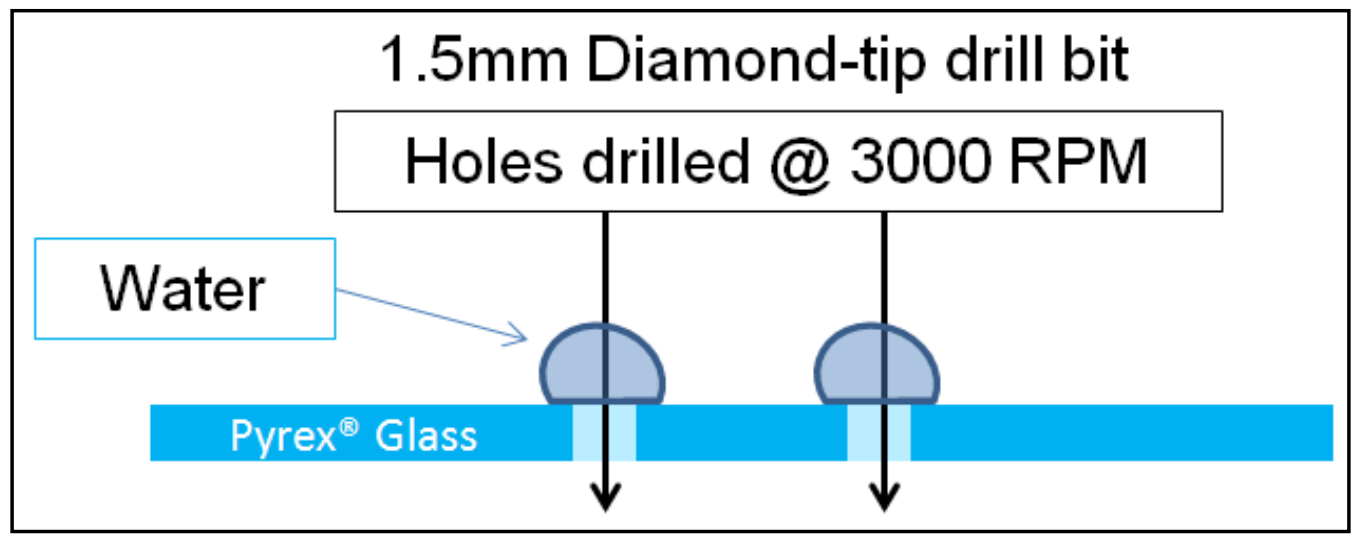

Figure 32 - Cross section showing how holes can be drilled through a Pyrex ${ }^{\circledR}$ wafer with minimal chipping around the edges

\subsubsection{Anodic Bonding}

The next processing step was to anodically bond the Pyrex (®) wafer to the silicon wafer. First, the silicon wafer was set down on the bottom aluminum block. Next, the Pyrex ${ }^{\circledR}$ wafer was set on top of the silicon wafer with care to make sure that the drilled holes lined up with the etched inlet and outlet holes on the silicon wafer. Finally, the top aluminum block was placed on top of the Pyrex ${ }^{\circledR}$ wafer (Figure 33). Since both the silicon and Pyrex® substrates are clean, they tended to slide against other when applying the top aluminum block; thus, altering the alignment. In those instances, the top aluminum block was removed and the substrates were rearranged correctly in the stack once again. 


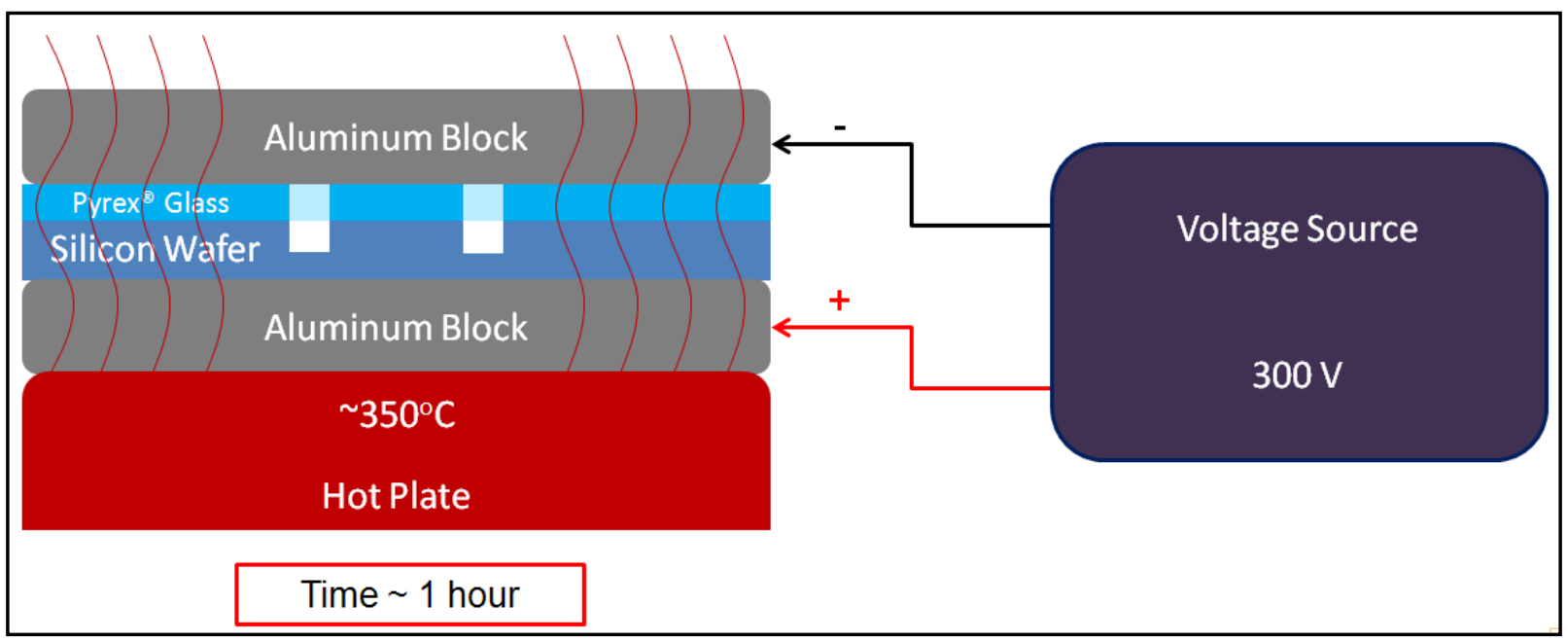

Figure 33 - Cross section showing the anodic bonding testing apparatus. The hot plate allowed for ion diffusion in the solid substrates, while the voltage was the driving force for anodic bonding to occur.

The anodic bonding process was completed by the following these procedures:

1) The stack was arranged in the sequence depicted in Figure 33. Care was taken to ensure that the glass wafer did not slide on the silicon wafer when the top aluminum block was set down because there was little friction between these two smooth surfaces.

2) The negative charge clip was attached to the top block and the positive charge clip was attached to the bottom block.

3) The hot plate was turned on to the maximum setting.

4) When the flashing temperature setting (540) stopped, the voltmeter was turned on and set to 300 volts.

5) After 120 minutes the voltmeter and hot plate were turned OFF.

6) Using tongs, the top aluminum block was carefully removed and set it down on the ceramic block next to the apparatus. Extra caution was necessary during this step because the glass sometimes got stuck to the aluminum block; therefore, the aluminum block was only lifted about $1 \mathrm{~cm}$ until it was ascertained that the wafer was not still clinging onto it. 
7) The bonded substrates were inspected and bonding completion was verified. (Air pockets were obvious). If not completely bonded, anodic bonding was repeated for additional time until complete bonding was finished.

The process parameters for anodic bonding are shown in Table IX.

Table IX - Process Parameters for Anodic Bonding

\begin{tabular}{|c|c|c|c|}
\hline Temperature, ${ }^{\circ} \mathrm{C}$ & $\begin{array}{c}\text { Pre-heat time, } \\
\text { minutes }\end{array}$ & Voltage, $\mathrm{V}$ & Time, minutes \\
\hline 380 & 10 & 300 & 120 \\
\hline
\end{tabular}

\subsubsection{Interfacing Syringes to the Microfluidic Reactor}

The next challenge in creating the microfluidic reactor was to interface the bulk CdSe solution from a syringe to the microfluidic channels. Interfacing was possible by using PDMS and a Duradyne Argon Plasma Surface Treatment Station (Figure 34).

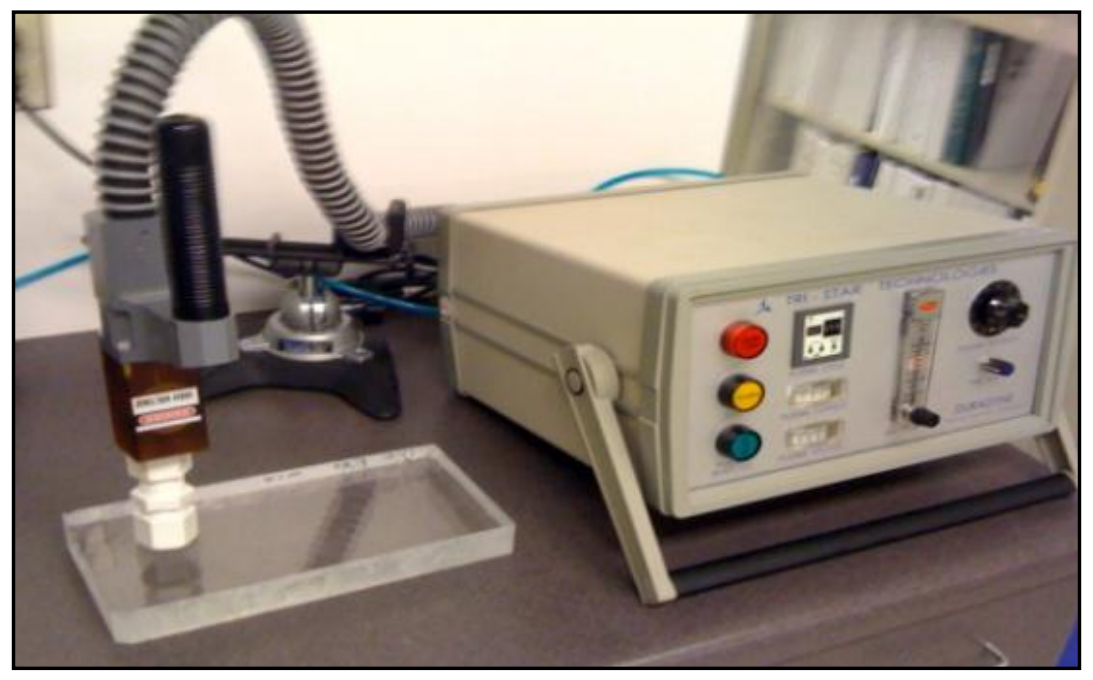

Figure 34 - Duradyne argon plasma system

The desired 7.5:1 ratio of mixing PDMS was determined by following the DOE described in [2.1.3 Design of Experiment (DOE)] (detailed results in Appendix A). The PDMS interface was created by the following procedures:

1) Using a syringe, $30 \mathrm{~mL}$ of PDMS base was deposited into a plastic cup. 
2) Using a different syringe, $4 \mathrm{~mL}$ of PDMS curing agent was added to the same plastic cup.

3) The base and curing agent were thoroughly mixed with a wooden stir stick.

4) The mixture was poured into a 3-inch polystyrene (PS) Petri dish.

5) The Petri dish was placed in a vacuum chamber and a vacuum and venting process was used to remove all the bubbles from the solution.

6) The degassed PDMS Petri dish was set in a pre-heated $70^{\circ} \mathrm{C}$ to cure the PDMS.

7) After 1 hour, the petri dish was removed from the oven and brought to room temperature equilibrium.

8) The PDMS was removed from the PS Petri dish and placed on a glass cutting table.

9) The PDMS was cut into approximately 1" by 1" squares.

10) Using a 16-gauge stainless steel needle, a hole was "punched" in the center of the PDMS square and the plug was removed (Figure 35). Care was taken to make sure that the act of punching the PDMS did not result in 'tears' in the PDMS.

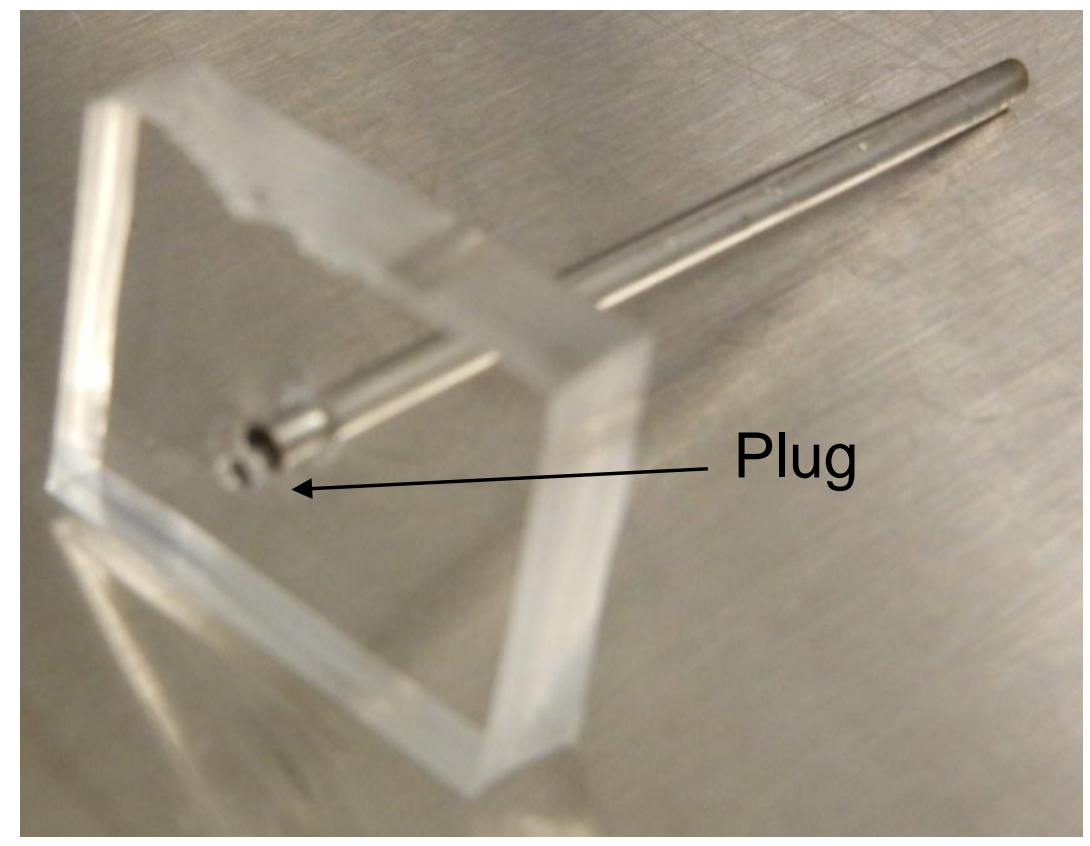

Figure 35 - SS316 tubing "punched" through PDMS results in a small plug that falls out of the other side. 
11) The SS316 tubing remained in the PDMS, while slight extraction was necessary to ensure that it was not hanging out past the face of the PDMS (Figure 36).

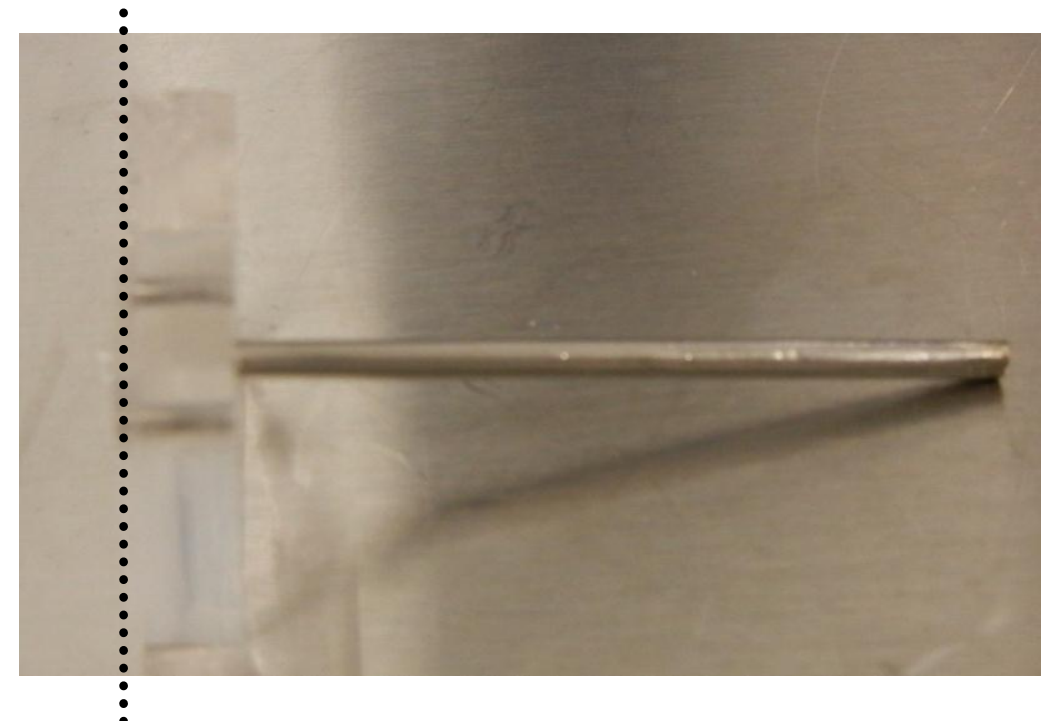

Figure 36 - SS316 Tubing inserted through the center of a PDMS block. The SS316 tube did not pass the outside face of the PDMS on the other side (bonding side).

12)The Duradyne Argon Plasma system was turned on and the high-purity argon gas bottle was opened.

13) The READY button (green) was pressed to turn on the plasma.

14) Using caution, the surfaces of the glass and PDMS were exposed to the argon plasma (Figure 37) in the following sequence:

a. 30 seconds glass surface

b. 60 seconds PDMS block

c. 30 seconds glass surface 


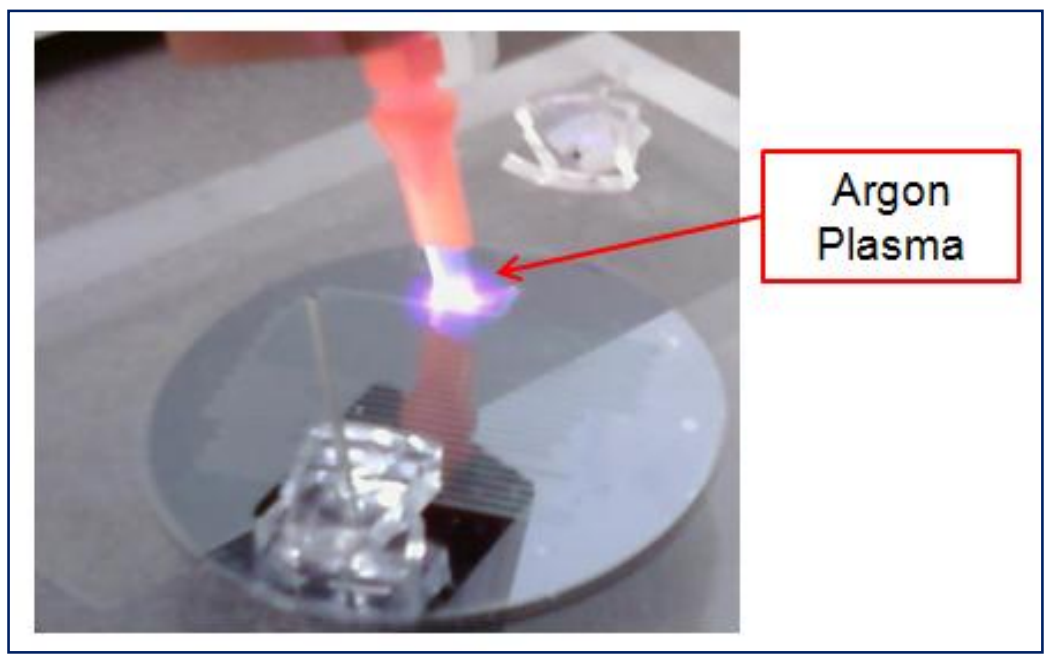

Figure 37 - Close-up of Argon plasma being applied to the surface of the Pyrex ${ }^{\circledR}$ wafer for the purposes bonding to PDMS

15) The plasma was turned off by pressing the PLASMA (yellow) button.

16) The hole in the PDMS was carefully aligned over the hole in the glass and gently pressed together. Care was taken to limit the size of the air pocket between the PDMS and glass.

17) The wafer/PDMS assembly was placed into the oven $\left(70^{\circ} \mathrm{C}\right)$ for about 10 minutes to finish the bonding process.

18) Steps 13-17 were repeated to put a PDMS chunk over the other hole.

19) The Duradyne Argon Plasma System was turned off and the argon gas bottle was closed.

The complete interface and cross-section diagram is shown in Figure 38. Full details of the pressure capabilities of the PDMS to glass interface is provided in Appendix A. 


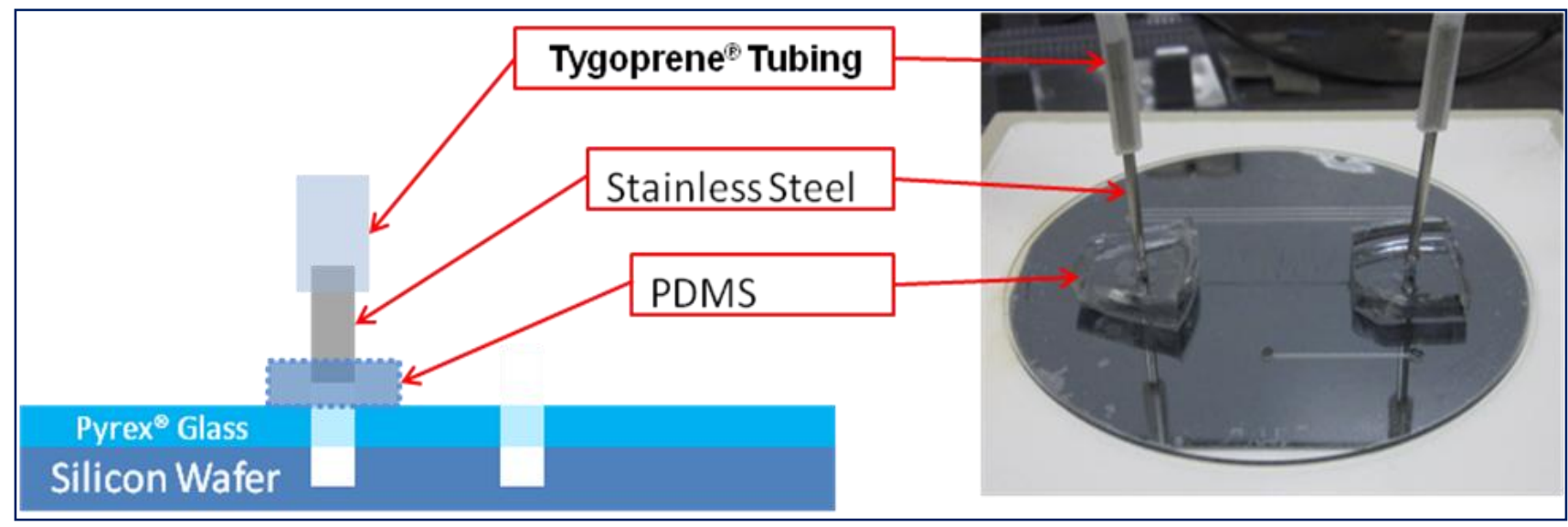

Figure 38 - Cross section of PDMS and microfluidic reactor showing interface material setup

\subsubsection{Testing Setup}

A syringe pump controlled the volumetric flow rate (pump rate) of the syringe holding the CdSe room temperature solution. Testing was setup using the following procedures:

1) The microfluidic reactor was set on a hot plate under a fume hood.

2) The syringe was connected to 6 inches of B-44-3 Tygon ${ }^{\circledR}$ tubing.

3) The other end of the Tygon ${ }^{\circledR}$ tubing was press-fit over the SS316 tubing in the PDMS plasma-bonded to the microfluidic reactor.

4) Another 6 inches of B-44-3 Tygon ${ }^{\circledR}$ tubing was press-fit over the other SS316 tube that led to a collection vial (Figure 39; Figure 40) on a box of equal height. 


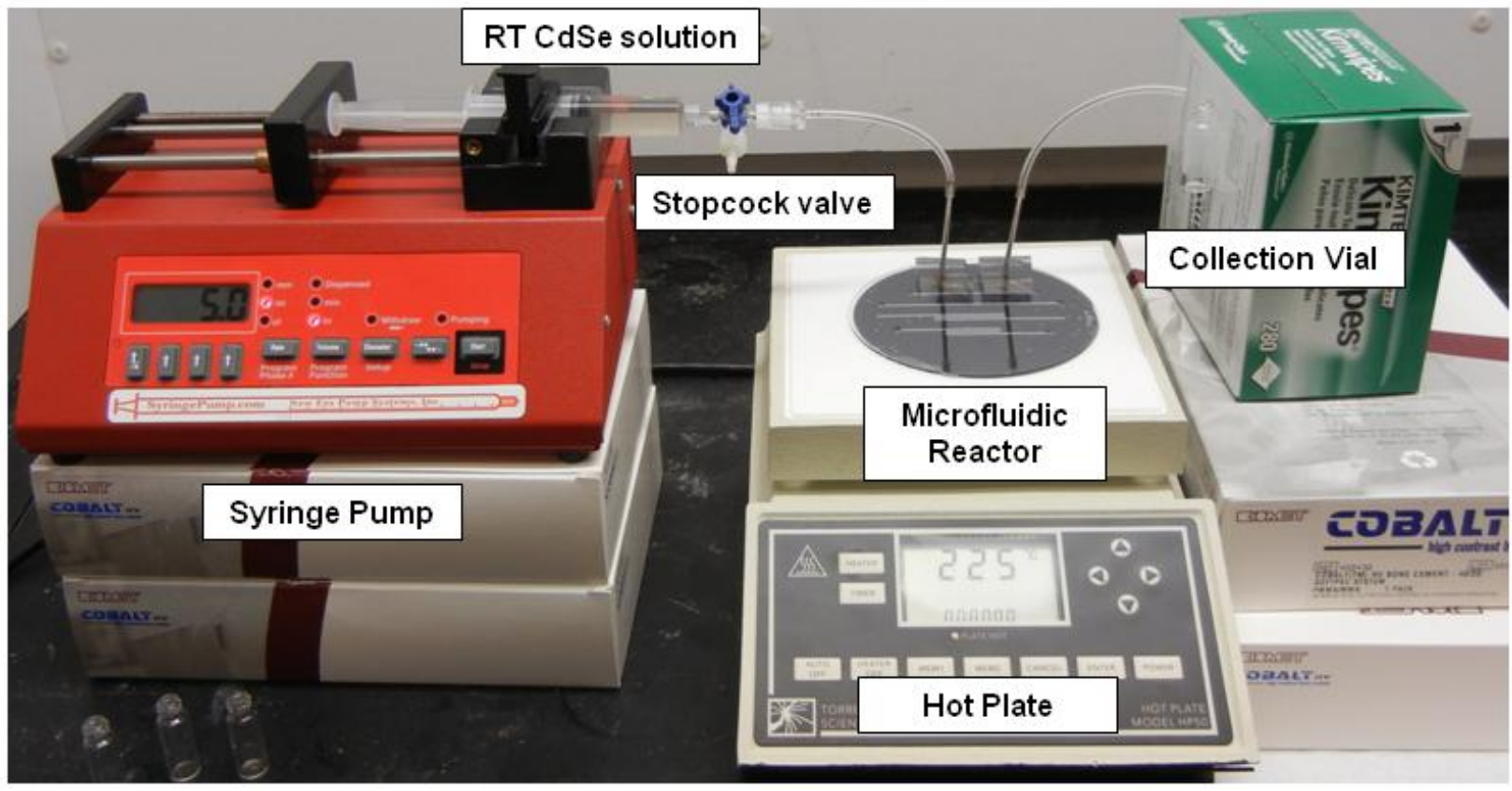

Figure 39 - Testing involved using a syringe pump to control the pump rate (Volumetric Flow Rate), a hot plate to control the temperature, and a small vial to collect synthesized CdSe quantum dots.

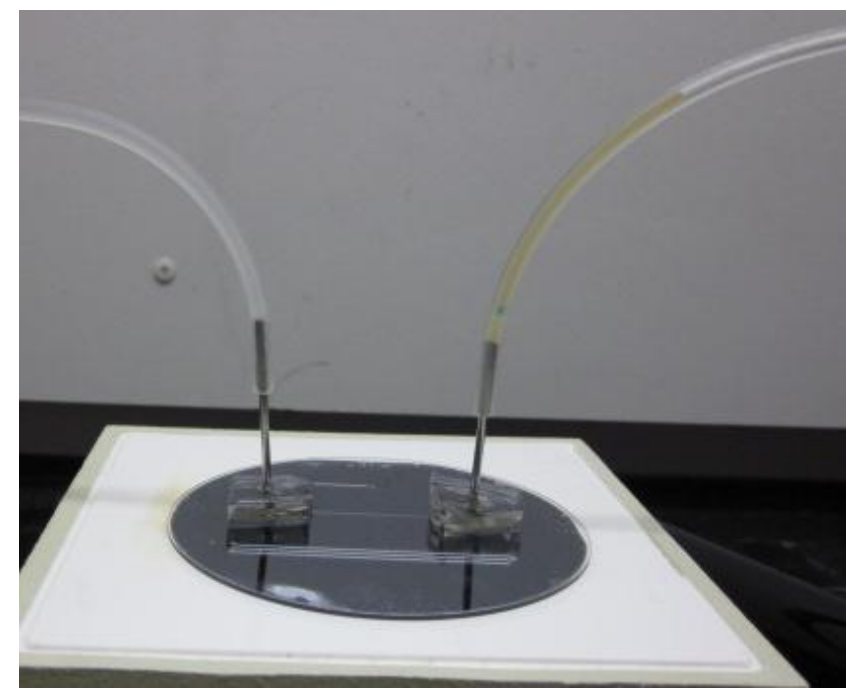

Figure 40 - Close-up of microfluidic device on the hotplate: the left tube contained a clear room temperature CdSe solution, while the right tube shows some color that indicated a chemical reaction had occurred on the hot plate.

5) The hot plate was turned on and set to $225^{\circ} \mathrm{C}$.

6) A black light was set up in order to observe fluorescence during testing (Figure 41). 


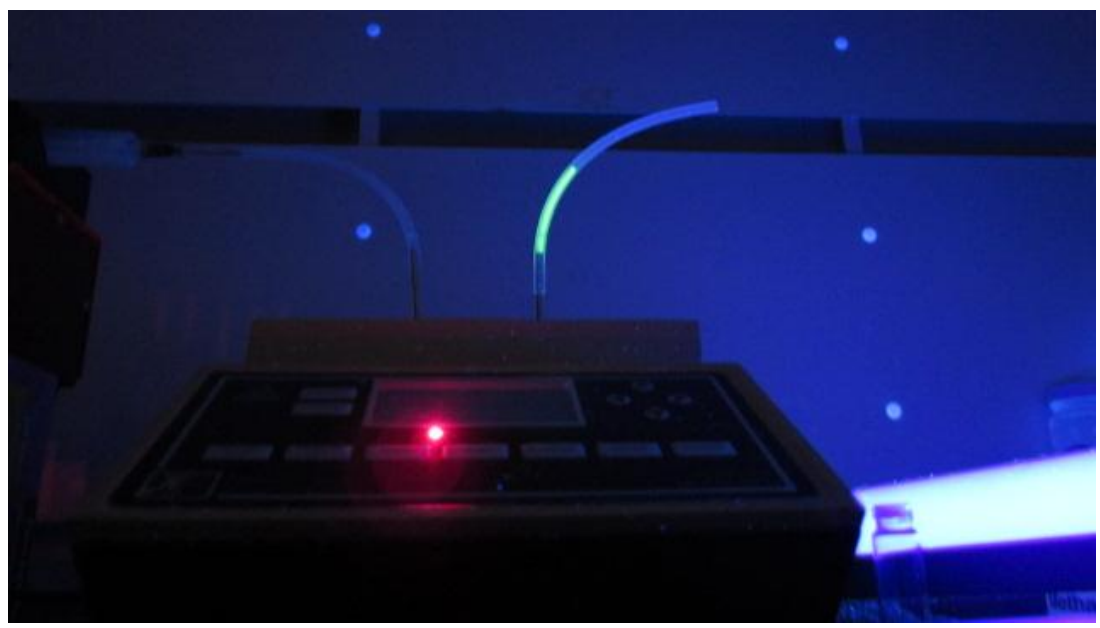

Figure 41 - Exposing the testing apparatus to a black light revealed that CdSe QDs were synthesized because of the fluorescence of the solution in the outlet tubing.

7) The Syringe Pump was turned on and the diameter was set to $14.80 \mathrm{~mm}$ (for the 12-cc syringe used during testing).

8) The pump rate was set to $5 \mathrm{~mL} / \mathrm{hr}$.

9) The procedure was continued until $1 \mathrm{~mL}$ was in the collection vial.

10) The pump was turned off and the stop cock valve was turned off to prevent solution in the Tygon ${ }^{\circledR}$ tubing or channels from back flowing into the syringe.

11) The hot plate was turned off and the microfluidic reactor was brought to room temperature.

12) Once at room temperature, the waste solution was pumped through the channels and tubing into a waste container.

13)The stop cock was removed from the apparatus.

14) Methanol was pumped through the tubing/channels to "clean" of residual QDs.

15) The Tygon ${ }^{\circledR}$ tubing was replaced for the next test.

16) The collected sample was put in a quartz cuvette and set in the sample holder to be exposed to a blue LED for analysis. 


\subsection{Characterization of Synthesized Quantum Dots}

Quantum dots have the unique material property of fluorescing when exposed to UV light. The color of fluorescence is an indicator of the size of the quantum dot; thus, not only does fluorescence testing confirm that QDs were synthesized within the channels of the microfluidic reactor, but it gives a measure of the approximate size of the QDs, as well. Fluorescence testing involves analysis of data to determine the central wavelength (CWL) and investigating the FWHM as a quantum dot size distribution.

\subsubsection{Fluorescence Testing}

Fluorescence testing was the main characterization technique used in determining the size of the quantum dots. Blue colors indicated the CdSe QDs were around $2 \mathrm{~nm}$ in size, while red QDs were around $5 \mathrm{~nm}$ in size. In order to test a QD sample, a small portion was placed in a quartz cuvette and the cuvette was exposed to an excitation light source. The resulting fluorescence was measured using an Ocean Optics USB4000 Spectrometer and software (Figure 42). 


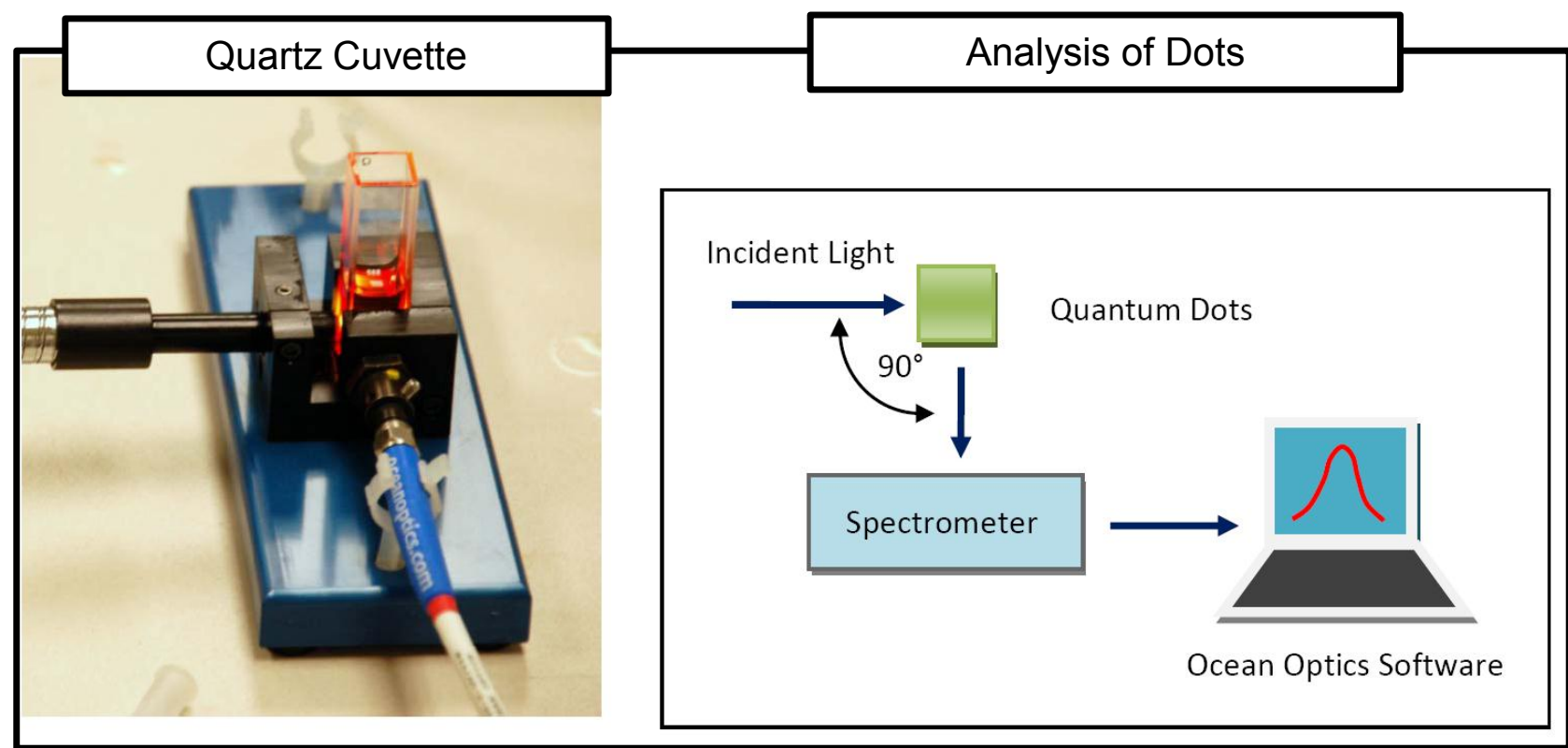

Figure 42 - Testing quantum dots for fluorescence involves putting a sample in a cuvette, exposing it to an excitation source (such as a blue LED), and measuring the resulting fluorescence with a spectrometer.

\section{Chapter 3 Results}

\subsection{Spectrum}

The spectrometer created a graph depicting wavelength vs. intensity. Testing indicates a relationship between pump rate and quantum dot size (Figure 43); however, this relationship was not investigated further because the goal of my Thesis Project was to fabricate a functioning microfluidic reactor. 


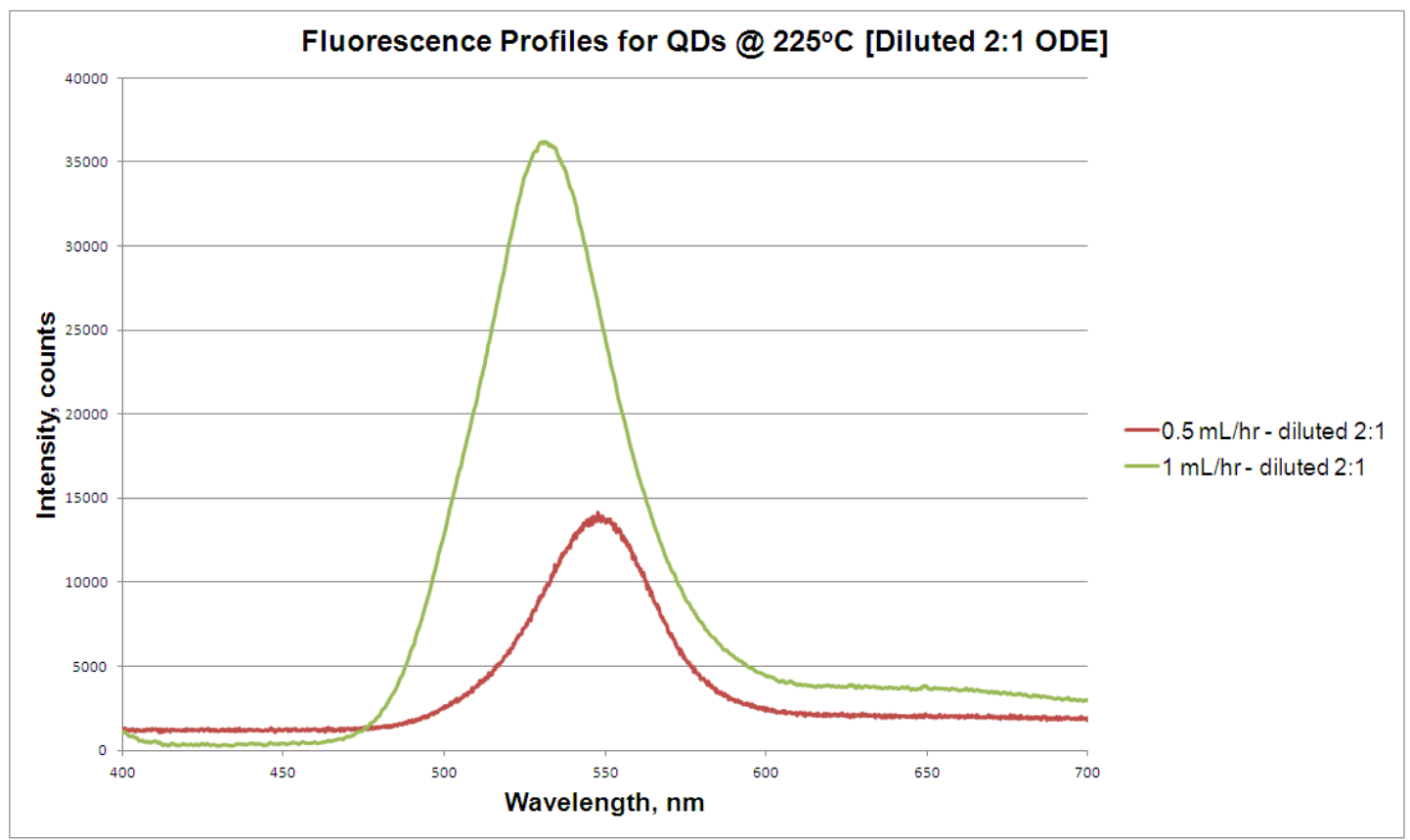

Figure 43 - Spectral profiles of two different QD syntheses indicating the trend that faster pump rate yielded smaller quantum dots; thus, shorter wavelength fluorescence.

Smaller QDs correlated to smaller wavelength fluorescence; however, this thesis project did not carry out a full DOE on the relationship between flow rate and wavelength.

\subsection{Repeatability and Reproducibility}

As part of this project, it was necessary to establish whether or not the microfluidic reactor was capable of repeatability and reproducibility. All synthesis reactions were completed at the $5 \mathrm{~mL} / \mathrm{hr}$ rate through the shortest channel $(2.5 \mathrm{~cm})$. Six tests were conducted in one day (Day 1) to establish repeatability. After one week elapsed, another six tests were conducted to establish reproducibility (Day 7). The results of these tests are shown in Table $\mathrm{X}$. 
Table X - Repeatability and Reproducibility Tests Results

\begin{tabular}{|c|c|c|}
\cline { 2 - 3 } \multicolumn{1}{c|}{} & $\begin{array}{c}\text { Peak }-\lambda, \mathrm{nm} \\
(\mathrm{CWL})\end{array}$ & FWHM, nm \\
\hline $\begin{array}{c}\text { Day 1 } \\
\text { Average }\end{array}$ & 492.7 & 38.2 \\
\hline $\begin{array}{c}\text { Day 1 } \\
\text { Standard Deviation }\end{array}$ & 1.63 & 4.92 \\
\hline $\begin{array}{c}\text { Day 7 } \\
\text { Average } \\
\text { Day 7 }\end{array}$ & 511.4 & 39.0 \\
\hline Standard Deviation & 1.01 & 9.14 \\
\hline
\end{tabular}

The repeatability showed little variation in the CWL from one test to the next; however, there exists a statistical difference between the CWLs during the reproducibility tests. A $p$-value of $<0.001$ confirms that the CWL are different. The FWHM values have a $p$ value of 0.085 , which suggests that they are not statistically different. Therefore, it can be said that QD synthesis is possible in the microfluidic reactor with repeatable results; however, further investigation was needed to identify the variation in tests from one CdSe batch to the next batch.

\section{Chapter 4 Discussion}

\subsection{Macroscopic vs. Microscopic}

The CdSe QD bulk synthesis method in the Cal Poly Nanotechnology Lab has been shown to achieve fluorescence values between $480 \mathrm{~nm}$ (blue-green) to $600 \mathrm{~nm}$ (red). The primary objective of this project was to achieve a functioning microfluidic reactor, defined as capable of synthesizing QDs, which was achieved. The next goal is to 
synthesize QDs that fluorescence below 480nm (blue) or above 600nm (red), in addition to producing a narrower FWHM.

\subsubsection{Can the Microfluidic Reactor Synthesize Blue or Red Light?}

Faster pump rates synthesize smaller QDs in the microfluidic reactor; however, there is also a corresponding increase in pressure. Pressure testing revealed the actual threshold pump rate that will minimize the residence time of the nucleation and growth reaction. It may be possible to experimentally achieve a blue fluorescence; however, it will be difficult to stop the reaction quickly enough to stop growth once out of the channel. Implementing a heat sink on the outlet tubing may assist in "quenching" the reaction or adding a second syringe pump to extract the solution should allow rapid synthesis.

In particular, there exists a large volume difference between the channels in the microfluidic reactor and the outlet tubing. As a result, the CdSe solution moves quickly through the channels; however, the volume of the solution accumulates on the hotplate below the outlet tubing. Only after a significant volume accumulates will the solution begin to move off the hotplate by way of the Tygon ${ }^{\circledR}$ tubing. The time that it takes for this solution to accumulate greatly adds on to residence time of the CdSe QDs. While not completely necessary to calculate the added residence time, it can be determined experimentally through iterations of QD synthesis. 
Conversely, it appears that slower pump rates will carry the growth reaction long enough to yield larger QDs (around $600 \mathrm{~nm}$ ); therefore, it should be possible to pump the CdSe solution through the microfluidic reactor slow enough to allow a similar result. An advantage of using a microfluidic reactor to synthesize CdSe QDs is the ability to better control the reaction environment and theoretically achieve more discrete FWHM spectral profiles, in addition to wavelengths that are not achievable on the bulk synthesis scale.

\subsubsection{The Tail for High Residence Times}

Faster pump rates yield CdSe QDs of smaller sizes; however, there exists a broad tail in the larger wavelength region of the graph (Figure 43). The tail occurs because there is a flow of continuous QD particle nucleation and growth. The tail correlates to a QD size distribution, such that there is a range of larger QDs fluorescencing at a lower intensity than the dominating peak of the solution. Removing the tail may be possible by inserting a heat sink on the microfluidic reactor to "quench" the growth reaction of the QDs as they emerge from the microfluidic channels. Another suggestion is to "filter" the QDs, such that particles over a certain size ( $4 \mathrm{~nm}$, for example) would be filtered from the batch solution, leaving the particles from the dominant CWL.

\subsubsection{Dilution Effects}

The relative intensity of fluorescence is around 5000 counts when the CdSe QDs are synthesized through the microfluidic reactor. The intensity can be increased as much as 10 times when diluted with additional octadecene. A 2:1 ratio of octadecene to CdSe 
QD solution appears to be ideal. The reason dilution increases the intensity is simply because by diluting the QDs, more light can fluoresce through the solution without interference by other QDs. Samples collected in this project were able to be diluted and the result was an increase in fluorescent intensity.

\subsubsection{Repeatability and Reproducibility}

Testing on Day 1 and Day 7 indicate the microfluidic reactor produces repeatable results, despite the small sample size. The area of concern for the reactor is difference in the reproducibility results between Day 1 and Day 7 . The difference may come from a flaw in the SOP for synthesizing precursor solutions that creates a difference in initial concentration of the room temperature CdSe solution.

The cadmium precursor was synthesized following the QD Synthesis SOP created by Aaron Lichtner [9]. The process asks for a continuous nitrogen purge; therefore, does not require users to remove the purge and vent needles at any point during the synthesis. Something I observed while synthesizing the cadmium precursor by following the SOP was that a significant amount of solution was evaporating out of the vent needle during heating of the flask. While waiting for the solution to become optically clear, different amounts of the solution evaporated. The length of time waiting for an "optically clear" solution is a matter of subjective opinion as to when exactly the solution has changed in clarity. Additionally, the vent needle is pumping a cool nitrogen gas into the vial, which essentially cools the environment within the chamber, which also affects how much time it will take to turn the solution optically clear. 
The amount that evaporated was directly proportional to the amount of time the flask was heated, which in turn varied with how quickly the hot plate was heating solutions that day. Prior to heating, the cadmium solution started out with $10.6 \mathrm{~mL}$ total volume (10 $\mathrm{mL}$ Octadecene and $0.6 \mathrm{~mL}$ of Oleic Acid). There was a large difference between Day 1 and Day 7's initial cadmium precursor volumes. Day 1 had about $7 \mathrm{~mL}$ of cadmium solution remaining, while Day 7 had only $5.5 \mathrm{~mL}$ available. The SOP required that $1 \mathrm{~mL}$ of selenide precursor get mixed with the $10.6 \mathrm{~mL}$ of the cadmium solution; however, my actual ratios were significantly different. The resulting difference in concentration likely affects the QD fluorescence wavelengths, though additional testing in this area is encouraged in future research projects.

To eliminate this problem, I synthesized the cadmium precursor again, but the vent and purge needles were removed prior to heating. The result was a $10.6 \mathrm{~mL}$ optically clear cadmium precursor solution. Making this change to the SOP resulted in the same initial volume of cadmium precursor, which assured that I had the same room temperature CdSe solution for each test.

The repeatability and reproducibility tests were carried out following this procedural change to the QD synthesis SOP and in all cases I achieved a central wavelength (CWL) of $492 \mathrm{~nm}$; thus, removing the vent and purge needles prior to heating the cadmium precursor solution allows for QD reproducibility through the microfluidic channels. Keep in mind these results are based on a small sample size completed after- 
the-fact. My thesis project ran out of time for sufficient testing; however, the project has been passed on to new students to continue to research and investigate.

\section{1 "Void" Residence Time}

One concern related of flowing solutions through a microfluidic reactor is the larger differences in volume between the inlet/outlet tubing, as compared to the microfluidic channels. In this project, the ratio of inlet tubing to channel volume was approximately 350:1. High pressures are associated with this volume change; however, I have compensated for this pressure and have shown that I am well the bursting strength of PDMS to glass (See Appendix A). A more significant problem is the "void" residence time of the synthesized QDs that are slowing filling the volume of the outlet tubing, yet still resting in the hot zone of the microfluidic reactor.

Simple calculations indicate that at $5 \mathrm{~mL} / \mathrm{hr}$, the residence time in a $25.7 \mathrm{um}$ deep channel is about 1 second. The same calculations show that it will take approximately 336 seconds to travel each length of the inlet and outlet Tygon® tubing. While in the microfluidic channel, it is safe to that the $\mathrm{QD}$ reaction is taking place at the maximum temperature within the microfluidic reactor, given a constant temperature on the hotplate.

There are no thermocouples located at any place on the outlet tubing. As a result, it is not possible to determine the temperature of the QDs in the outlet tubing at a given distance away from the microfluidic channel. Calculations indicate the length of times it 
takes for the solution to reach distances in the outlet tubing, which I call the "void" residence time (Table XI). In this time frame, I believe the QDs are decreasing in temperature, which correlates to a decrease in nucleation and growth rates; however, this period of time may also assist in the creation of the tail observed in fluorescence. Additionally, the "void" residence time may limit the ability of the microfluidic reactor to synthesize CWL in the blue wavelength region of the visible spectrum.

Table XI - "Void" Residence Times

\begin{tabular}{|c|c|}
\hline $\begin{array}{c}\text { Void distance, } \\
\text { inches }\end{array}$ & Res Time, $\mathbf{s}$ \\
\hline 0.5 & 24 \\
\hline 1 & 48 \\
\hline 2 & 96 \\
\hline 3 & 144 \\
\hline 4 & 192 \\
\hline 5 & 240 \\
\hline 7 & 336 \\
\hline
\end{tabular}




\section{Chapter 5 Conclusions}

Overall, this microfluidic research project had numerous successes; however, new questions have surfaced, in addition to a multitude of future research recommendations.

It has been shown that microfabrication techniques can etch microfluidic channels into silicon wafers. Further, holes can be wet-drilled through Pyrex ${ }^{\circledR}$ wafers by using a diamond-tip drill bit at 3000 RPM. Finally, silicon and glass substrates can be anodically bonded together to complete the walls of the microfluidic channels.

PDMS suffices as an interface between a bulk syringe solution and the microfluidic channels of a reactor. Pressure limits can be controlled by using short channel lengths (less than $12.5 \mathrm{~cm})$, low pump rates $(\sim 5 \mathrm{~mL} / \mathrm{hr})$, and a 7.5:1 ratio of PDMS. A significantly higher pressure can be achieved than was reached in this project.

The cadmium selenide solution can be mixed at room temperature and treated as a single solution for injection into a microfluidic reactor, so long as it is used shortly after precursor synthesis. The fluorescence spectral profiles reveal that FWHM is on a level that is comparable with commercially produced fluorescence profiles. Narrower FWHM may be achievable if the nucleation and growth reaction can be controlled (halted) by continuing to modify the microfluidic reactor (i.e. incorporating a heat sink on the reactor or using two syringe pumps.) 
This project focused on one temperature, one flow rate, and one channel length. I was able to consistently achieve the same CWL $(492 \mathrm{~nm})$ and FWHM $(\sim 35 \mathrm{~nm})$ repeatedly the same day (repeatability) and with a different batch a week later (reproducibility). Future research should be able to characterize the effects of changing the parameters that were held constant in my project. 


\section{Chapter 6 Future Work and Recommendations}

I recommend a full DOE is carried out that compares channel length, channel depth, pump rate, and hot plate temperature to determine their interactions with one another and how they affect the wavelength and FWHM of the synthesized quantum dots.

A second syringe pump should be implemented on the outlet tubing to extract solution from the channels at the same rate as the pump rate. This will allow users to rapidly push/pull solutions on the microfluidic reactor and possibly narrow the FWHM by allowing QDs to be cooled faster. Further, a heat sink on the microfluidic reactor may ensure the CdSe QD reaction will cease; thus, reducing the FWHM and possibly eliminating the "tail" on the fluorescence profile.

I suggest the creation of two channels to accommodate the cadmium and selenide precursors as separate injections, such that they will meet and mix on the chip. Due to laminar flow, this project avoided mixing problems by combining the solutions at room temperature; however not all chemical reactions will allow the same luxury. The device should be designed to force the solutions to mix within the channels.

During drilling of the holes in the glass wafer, I would suggest beginning drilling on one side (anodic bonding side), then invert the glass wafer and finish drilling from the other side. This technique should prevent glass chipping from occurring on either side of the Pyrex ${ }^{\circledR}$ wafer. Further, I suggest that the microfluidic reactor is fabricated to 
accommodate thermocouples which can identify the actual temperature of the CdSe solution in the microfluidic channels.

Now that the principle of creating a functioning microfluidic reactor has been proven successful, I suggest a mask design with smaller channel widths than the 1000 microns used in this project. Smaller widths should correlate to tighter control over the reaction conditions. 


\section{Appendix A PDMS Ratio Testing}

\section{Background}

One of the largest obstacles to an operational microfluidic reactor was to ensure the variables kept the pressure in the reactor below the bursting strength of the PDMSPyrex ${ }^{\circledR}$ bond. The bond strength of PDMS to Pyrex ${ }^{\circledR}$ has been shown to be around 25 psi [45]. Further, the manufacturer recommends a ratio of $10: 1$ be used in synthesizing the PDMS.

The secondary DOE was designed to investigate the 10:1 ratio of base to curing agent, and to determine ways to possibly reduce the probability of device failure due to the interface between the PDMS and glass substrates. Testing was necessary to determine the relationship between ratio (base: curing agent) with bursting strength to see what, if any, trend exists and to possibly modify the factory recommended 10:1 ratio for implementation in a microfluidic reactor, such as this.

\section{Materials}

The PDMS synthesized in this project is Sylgard® 184 , which is a two component system. The base and curing agent are mixed together and stirred. During the stirring process, polymerization occurs and networks and cross-links are formed. Finally, the material is cured at an elevated temperature to complete the reaction. 
The components of the Sylgard® 184 Silicone Elastomer Base are:

- Dimethyl siloxane, dimethyl vinyl-terminated (CAS 68083-19-2)

- Dimethyl vinylated and trimethylated silica (CAS 68988-89-6)

- Tetra (trimethyl siloxy ) silane (CAS 3555-47-3)

- Ethyl benzene (CAS 100-41-4)

The components of the Sylgard 184® Silicone Elastomer Curing Agent are:

- Dimethyl, methyl hydrogen siloxane (CAS 68037-59-2)

- Dimethyl siloxane, dimethyl vinyl-terminated (CAS 68083-19-2)

- Dimethyl vinylated and trimethylated silica (CAS 68988-89-6)

- Tetra methyl tetra vinyl cyclotetra siloxane (CAS 2554-06-5)

- Ethyl benzene (CAS 100-41-4)

After mixing the two (suggested wt. ratio 10:1), the curing process starts, which crosslinks prepolymers found in both components, turning the liquid into rubber. The main chemical constituent of the base mixture is dimethyl vinyl-terminated dimethyl siloxane (more than $60 \mathrm{wt} \%$ of base), whereas the curing agent is made of dimethyl methyl hydrogen siloxane (40-70 wt\% of curing agent) [28]. The reaction cross-links the methyl hydrogen siloxane units (from the curing agent) with the terminal vinyl groups of the base polymers, turning short liquid polymer chains into an elastomeric network [46].

PDMS bonds to PDMS or glass using oxygen or argon plasma. Bonding occurs due to the condensation reaction between silanol groups, when plasma treated surfaces are pressed together (Figure 17) [31]. 


\section{Procedures}

The testing apparatus was personally designed for this project and does not follow any ASTM standard. The equipment that was purchased specifically for this testing was a Pasco PS-2107 Gas Pressure Sensor because it has a range from 0 to $700 \mathrm{kPa}(\sim 125$ psi). Also used in this experiment was a Pasco Datalogger (which used Data Studio software).

PDMS was synthesized following the standard operating procedure in the ratios in Table A I).

Table A I - Tested Mixture Ratios of PDMS Base to Curing Ag
\begin{tabular}{|c|c|c|}
\hline Ratio & Volume Ratio, mL & Total Volume, mL \\
\hline $5: 1$ & $26: 5.2$ & 31.2 \\
\hline $7.5: 1$ & $30: 4$ & 34 \\
\hline $10: 1$ & $30: 3$ & 33 \\
\hline $15: 1$ & $30: 2$ & 32 \\
\hline $20: 1$ & $30: 1.5$ & 31.5 \\
\hline
\end{tabular}

Each test was completed using the following procedure:

1) Plasma bond and cure a PDMS square chunk to the clean, dry surface of a Pyrex® wafer.

2) Set-up the Syringe pump such that the fluid flows through a valve that accommodates both the gas pressure sensor and access toward the PDMS/Glass interface (Figure A 1). 


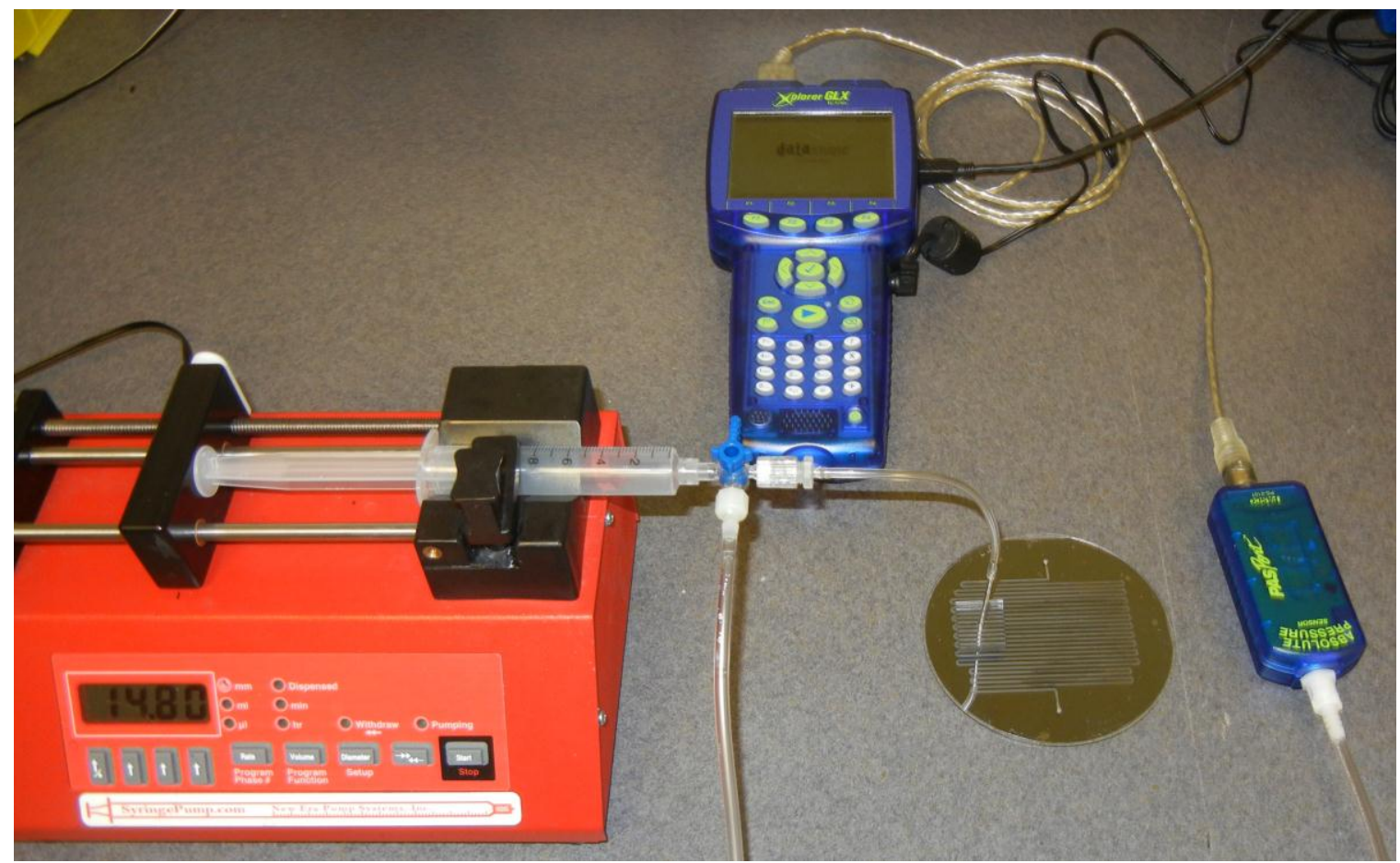

Figure A 1 - Test Apparatus to measure pressure necessary to break the PDMS/Glass interfacial bond. A syringe pump pushes fluid down into two Tygon ${ }^{\circledR}$ tubes, one toward the bond interface and the other toward the gas pressure sensor.

3) Turn on the Data Logger software.

4) Set up the appropriate pump rate that suits the limits of the syringe being used. (For example, the $12-c c$ syringe could use $50 \mathrm{~mL} / \mathrm{hr}$ pump rate, while the $30 \mathrm{~mL}$ syringe could use the $100 \mathrm{~mL} / \mathrm{hr}$ pump rate). The faster the pump rate, the greater the pressure will increase and the quicker the testing will take place.

5) Fill the syringe with water.

6) Start the Data Logger and the Syringe Pump at the same time and let the test run its course.

7) Take paper towels and place around the interface because when the bond fails, water will squirt violently in unpredictable direction (such as towards the computer equipment!) (Figure A 2) 


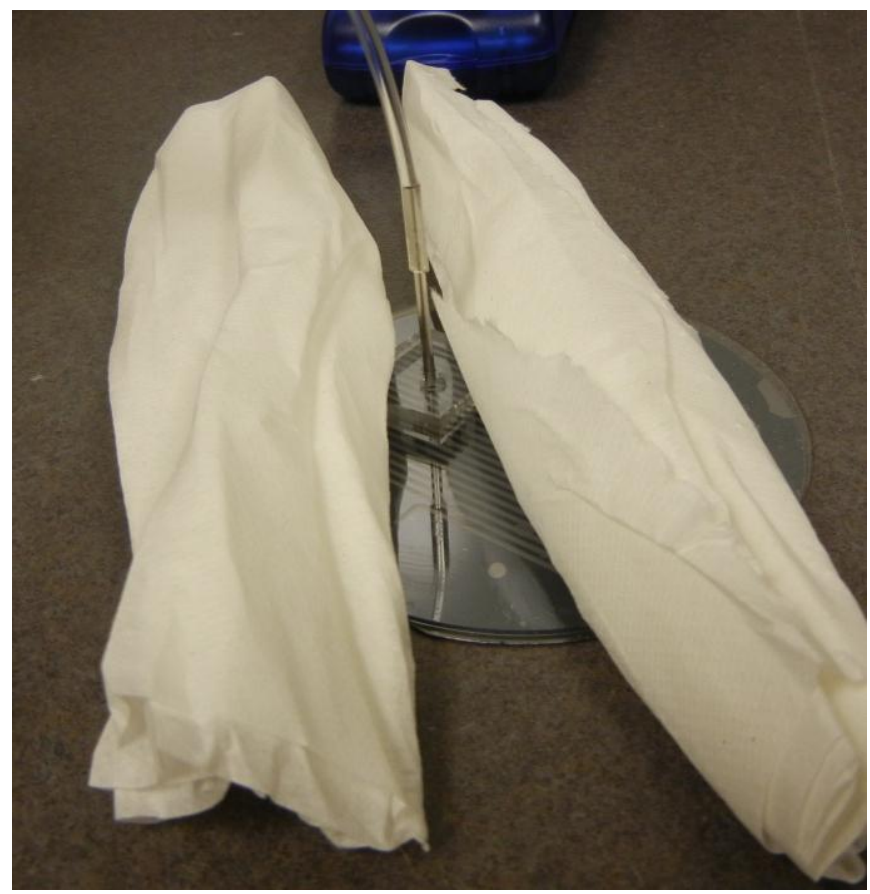

Figure A 2 - Paper towels will absorb most of the water that rushes from the microfluidic device when the PDMS/Glass interfacial bonding fails.

8) Once the test is complete, repeat four more times for each ratio (following the DOE designed in Chapter 2 of the Thesis Report).

\section{Results}

Testing reveals that the 7.5:1 ratio creates the highest average bursting strength (Table A II).

Table A II - Bursting Strengths of PDMS to Glass with Different PDMS Mixture Ratios

\begin{tabular}{|c|c|c|c|c|c|c|}
\hline \multicolumn{7}{|c|}{ PDMSlass Bursting Strength } \\
\hline Ratio & $\mathbf{5 : 1}$ & $\mathbf{7 . 5 : 1}$ & $\mathbf{1 0 : 1}$ & $\mathbf{1 5 : 1}$ & $\mathbf{2 0 : 1}$ & Units \\
\hline Run 1 & 515.0 & 517.1 & --- & --- & --- & $\mathrm{kPa}$ \\
\hline Run 2 & 441.2 & 564.0 & 486.4 & 328.4 & 192.0 & $\mathrm{kPa}$ \\
\hline Run 3 & 524.0 & 486.7 & 364.0 & 333.2 & 175.1 & $\mathrm{kPa}$ \\
\hline Run 4 & 448.6 & 541.5 & 484.8 & 263.7 & 231.0 & $\mathrm{kPa}$ \\
\hline Average (kPa) & 482.2 & 527.3 & 445.1 & 308.4 & 149.5 & $\mathrm{kPa}$ \\
\hline Average (psi) & 69.96 & 76.50 & 64.57 & 44.75 & 21.69 & $\mathrm{psi}$ \\
\hline
\end{tabular}




\section{Discussion}

Several anomalies presented themselves during testing. In particular, the syringe pump would often struggle to pump when it approached 50 psi. In those instances, assisting the pump by applying manual pressure seemed to stop the problem. Additionally, leaking would often occur at high pressure at the interfaces of the Tygon/SS316 and SS316/PDMS. In these instances, the pressure was clearly affected in that there was relief evident. In all instances where the device failed at an interface other than the PDMS/Glass interface, the data was considered an outlier and the retested. This was necessary because I wanted to test the PDMS/Glass bonding strength specifically.

The trend indicates that an increase in ratio leads to a decrease in overall bonding strength. The 5:1 ratio may have the potential to have the highest bonding strength; however, the material is very stiff. In fact, the stiffness seems to outweigh the viscoelastic properties of PDMS. The viscoelasticity of PDMS is necessary to hold the SS316 in place. At the 5:1 ratio, numerous tests failed because the SS316 was forced out of the PDMS chunk. This is what led to deciding that the $7.5: 1$ ratio was the ideal ratio for the microfluidic reactor application.

The decreasing trend of bond strength can be explained by looking at the silane constituents in the base component. Silane is typically used to create more cross-linking sites; thus, as we increase the ratio of base we are increasing the level of cross-linking. More importantly, the cross-linking is localized, while the overall polymer is a network of crosslinks. The resulting trend with PDMS samples is that we see an increase in 
flexibility. Greater localized cross-linking results in a more flexible polymer, which supports the trend we observe. 


\section{References}

[1] Schmid, G. Nanoparticles: From Theory to Application. New York: John Wiley \& Sons, Incorporated, 2004. Print.

[2] Magee, Greg, and Jessie Yeates. "Nanomedicine." PBWorks.com. 2007. Web. Mar. 2010. <http://chem3513-2007.pbworks.com/w/page/15648426/Nanomedicine>.

[3] "Molecular Orbital Theory." Lecture. Grandinetti.org. Grandinetti Laboratory. Web. 3 Apr. 2011. <http://www.grandinetti.org/Teaching/Chem121/Lectures/MOTheory/?vm=r>.

[4] Zumdahl, Steven S. Chemistry Principles. 4th ed. Boston: Houghton Mifflin, 2001. Print.

[5] Pradeep, T. Nano: the Essentials: Understanding Nanoscience and Nanotechnology. New York: McGraw-Hill, 2008. Print. Professional.

[6] "How Quantum Dots Work." Evidenttech.com. Evident Technologies. Web. 7 Oct. 2010. <http://www.evidenttech.com/quantum-dots-explained/how-quantum-dotswork.html>.

[7] Salisbury, David F. "Quantum Dots That Produce White Light Could Be the Light Bulb's Successor." Vanderbilt News. Vanderbilt.edu, 20 Oct. 2005. Web. $<$ http://news.vanderbilt.edu/2005/10/quantum-dots-that-produce-white-light-could-bethe-light-bulbs-successor-59204/>.

[8] Weisstein, Eric W. "Gaussian Function." Mathworld.wolfram.com. Wolfram Research. Web. 29 Nov. 2011. <http://mathworld.wolfram.com/GaussianFunction.html>.

[9] Lichtner, Aaron. Process Development and Characterization of Cadmium Selenide Quantum Dots Synthesis through Nanoparticle Size Optimization. California Polytechnic State University, 5 Jan. 2009. Print.

[10] Uehara, Masato, Hiroyuki Nakamura, and Hideaki Maeda. "Preparation of ZnS/CdSe/ZnS Quantum Dot Quantum Well by Using a Microfluidic Reactor." Journal of Nanoscience and Nanotechnology 9.1 (2009): 577-83. Print.

[11] Ashby, Michael. Materials: Engineering, Science, Processing and Design. Boston: Butterworth-Heinemann, 2004. Print.

[12] Hummel, Rolf E. Understanding Material Science. 2nd ed. New York: Springer, 2004. Print.

[13] Watts, Paul, and Charlotte Wiles. "Recent Advances in Synthetic Micro Reaction Technology." Chemical Communications 5 (2007): 443-67. Print.

[14] Benson, Tom. "Reynolds Number." Grc.nasa.gov. National Aeronautics and Space Administration, May 2009. Web. 29 Nov. 2011.

<http://www.grc.nasa.gov/WWW/BGH/reynolds.html>.

[15] Roberson, Michael. The Design and Manufacture of a Microreactor to Synthesize CdSe Quantum Dots." California Polytechnic State University, 10 June 2010. Print. 
[16] Bhushan, Bharat, ed. Springer Handbook of Nanotechnology. 2nd ed. New York: Springer, 2007. Print.

[17] "Physical Vapor Deposition by Sputtering." SiliconFarEast.com. Web. Oct. 2011. <http://www.siliconfareast.com/sputtering.htm>.

[18] "Deposition Processes." Mems-exchange.org. MEMS and Nanotechnology Exchange. Web. Oct. 2011. <http://www.mems-

exchange.org/MEMS/processes/deposition.html>.

[19] Shipley Microposit S1800 Series Photoresists Data Sheet. Newton, MA: Shipley, 1993. Print.

[20] Jaeger, Richard C. "Lithography." Introduction To Microelectronic Fabrication. 2nd ed. Upper Saddle River, NJ: Prentice Hall, 2002. Print.

[21] "Etching Processes." Memsnet.org. MEMS and Nanotechnology Exchange. Web.

Oct. 2011. <http://www.memsnet.org/mems/processes/etch.html>.

[22] Flamm, Daniel. "Mechanisms of Silicon Etching in Fluorine- and Chlorine-containing Plasmas." Pure \& Applied Chemistry 62.9 (1990): 1709-720. Print.

[23] Wiemer, M., J. Fromel, and T. Gebner. "Trends in the Technological Development of Wafer Bonding." Chemnitzer Trade Conference Micromechanics \& Microelectronics 29/30.10 (2003): 178-188. Print.

[24] Gessner, T., T. Otto, and M. Wiemer. "Wafer Bonding in Micro Mechanics and Microelectronics - an Overview." The World of Electronic Packaging and System Integration (2005): 307-13. Print.

[25] Mack, S. Eine Vergleichende Untersuchung Der Physikalisch-chemischen Prozesse an Der Grenzschicht Direkt Und Anodischer Verbundener Festkörper. Rep. VDI, 1997. Print.

[26] Thomas, Berthold, and Karin Herzog. "Ion Drift Processes in Pyrex-Type AlkaliBorosilicate Glass during Anodic Bonding." Solar Energy 145.5 (1998): 1755-762. Print.

[27] Linear Polydimethylsiloxanes. Publication no. 26. Joint Assessment of Commodity Chemicals, September 1994. Print.

[28] Sylgard 184 Silicone Elastomer Data Sheet. Dow Corning, 2007. Print.

[29] Bodas, Dhananjay, and Chantal Khan-Malek. "Hydrophilization and Hydrophobic Recovery of PDMS by Oxygen Plasma and Chemical Treatment-An SEM Investigation." Sensors and Actuators B: Chemical 123.1 (2007): 368-73. Print.

[30] Sharma, Vikash, Marshal Dhayal, S. M. Shivaprasad, and S. C. Jain. "Surface Characterization of Plasma-treated and PEG-grafted PDMS for Micro Fluidic Applications." Vacuum 81.9 (May 2007): 1094-100. Print.

[31] Bhattacharya, Shantanu, Arindom Datta, Jordan M. Berg, and Shubhra Gangopadhyay. "Studies on Surface Wettability of Poly(Dimethyl) Siloxane (PDMS) and Glass Under Oxygen-Plasma Treatment and Correlation With Bond Strength." Journal of Microelectromechanical Systems 14.3 (June 2005): 590-97. Print. 
[32] Lee, Jessamine N., Cheolmin Park, and George M. Whitesides. "Solvent Compatibility of Poly(dimethylsiloxane)-Based Microfluidic Devices." Analytical Chemistry 75.23 (2003): 6544-554. Print.

[33] Savage, Richard N., Hans Meyer, Matthew Lewis, and Dan M. Marrujo. 2008 Materials Research Society Fall Meeting: Symposium M. Proc. of Utilizing Quantum Dots to Enhance Solar Spectrum Conversion Efficiencies for Photovoltaics. December 2008. Print.

[34] Service, Robert F. "Shortfalls in Electron Production Dim Hopes for MEG Solar Cells." Science 322 (2008): 1784. Sciencemag.org. AAAS, 19 Dec. 2008. Web. 29 Nov. 2011. <http://www.sciencemag.org/content/322/5909/1784.2.full.pdf>.

[35] Talbot, David. "TR10: Nanocharging Solar." Signallake.com. Technology Review, 12 Mar. 2007. Web. 29 Nov. 2011.

<http://www.signallake.com/innovation/QuantumDotSolarCell031207.pdf>.

[36] Walling, Maureen A., Jennifer A. Novak, and Jason E. Shepard. "Quantum Dots for Live Cell and In Vivo Imaging." International Journal of Molecular Sciences 10.2 (2009): 441-91. Print.

[37] Michalet, X., F. F. Pinaud, L. A. Bentolila, J. M. Tsay, S. Doose, J. J. Li, G. Sundaresan, A. M. Wu, S. S. Gambhir, and S. Weiss. "Quantum Dots for Live Cells, in Vivo Imaging, and Diagnostics." Science 307.5709 (2005): 538-44. Print.

[38] Ballou, Byron, B. C. Lagerholm, Lauren A. Ernst, Marcel P. Bruchez, and Alan S. Waggoner. "Noninvasive Imaging of Quantum Dots in Mice." Bioconjugate Chemistry 15.1 (2004): 79-86. Print.

[39] "New Instruments at UBuffalo Will Help Scientists Map Tumor Surfaces, Study Environmental Impact of Quantum Dots." Nanowerk News. Nanowerk.com, 10 Feb. 2011. Web. 29 Nov. 2011. <http://www.nanowerk.com/news/newsid=20097.php?vm=r>.

[40] "Quantum Dots \& Phosphor Dots Comparison Chart." Nanomaterialstore.com. Sun Innovations, Inc. Web. 7 Oct. 2010. <http://www.nanomaterialstore.com/nanophosphor.php>.

[41] "Cadmium-free Quantum Dots "CFQD"' Nanocotechnologies.com. Nanoco Group PLC. Web. 8 Oct. 2010.

<http://www.nanocotechnologies.com/content/AdvancedMaterials/CadmiumFreeQuantu mDotsQFQDHeavyMetalFree.aspx>.

[42] Safety Data for Zinc Sulfide. Oxford. Physical and Theoretical Chemistry Lab. Oxford University, 11 Sept. 2006. Web. 8 Oct. 2010. <http://msds.chem.ox.ac.uk/Zl/zinc_sulfide.html>.

[43] Pelley, Jennifer L., Abdallah S. Daar, and Marc A. Saner. "State of Academic Knowledge on Toxicity and Biological Fate of Quantum Dots." Toxicological Sciences an Official Journal of the Society of Toxicology 112.2 (2009): 276-96. Print. 
[44] Choi, H. S., W. Liu, P. Misra, E. Tanaka, J. P. Zimmer, Ipe B. Itty, M. G. Bawendi, and J. V. Frangioni. "Renal Clearance of Quantum Dots." Nature Biotechnology 25.10 (2007): 1165-170. Print.

[45] Bhattacharya, Shantanu. Plasma Bonding of Poly (dimethyl) Siloxane and Glass Surfaces and Its Application to Microfluidics. Thesis. Texas Tech University, 2003. Texas: Texas Tech University Libraries, 2003. Print.

[46] Hayashi, T. Comprehensive Asymmetric Catalysis. Ed. H. Yamamoto. Vol. 1. Berlin: Springer-Verlag, 1999. Print. I-III. 